\title{
1 Distinct dopamine circuits transmit the reinforcing and anxiogenic effects \\ 2 of nicotine
}

\section{Authors}

4 Nguyen $\mathrm{C}^{1}$, Mondoloni $\mathrm{S}^{1}$, Centeno $\mathrm{I}^{1}$, Durand-de Cuttoli $\mathrm{R}^{1}$, Tolu $\mathrm{S}^{1}$, Valverde $\mathrm{S}^{1}$, Le Borgne $\mathrm{T}^{1}$,

5 Hannesse $B^{1}$, Pons $S^{2}$, Maskos U2 , Dalkara $D^{3}$, Hardelin JP1 , Mourot $A^{1}$, Marti $F^{1 \#^{*}}$ \& Faure $P^{1 \#^{*}}$

\section{Affiliations}

71 Sorbonne Université, Inserm, CNRS, Neuroscience Paris Seine - Institut de biologie Paris Seine (NPS - IBPS),

875005 Paris, France.

92 Institut Pasteur, Unité Neurobiologie intégrative des systèmes cholinergiques, Département de neuroscience, 1075724 Paris cedex, France.

$11{ }^{3}$ Sorbonne Université, Inserm, CNRS, Institut de la Vision, Paris, France.

12 \# equal contributions

13 *Correspondence to fabio.marti@upmc.fr, phfaure@gmail.com

\section{Summary}

15 Nicotine, the addictive component of tobacco, stimulates dopamine (DA) neurons of the ventral tegmental 16 area (VTA) to establish and maintain reinforcement. Nicotine also induces negative emotional states such 17 as anxiety, yet through an unknown circuitry. Here we show that nicotine at reinforcing doses drives opposite functional responses on two distinct populations of VTA DA neurons with anatomically segregated projections: it activates those that project to the nucleus accumbens (NAc) while it inhibits those that project to the amygdala nuclei (Amg). We further show that nicotine, by acting on $\beta 2$ subunitcontaining nicotinic acetylcholine receptors of the VTA, mediates both reinforcement and anxiety. Finally, using optogenetic experiments we dissociate the roles of the VTA-NAc excitation and VTA-Amg inhibition in reinforcement and anxiety-like behavior, respectively. We thus propose that the positive and negative behavioral outcomes of nicotine consumption involve distinct subpopulations of VTA DA neurons with opposite responses to nicotine.

27 nicotinic acetylcholine receptors, dopamine circuits, addiction, juxtacellular recordings, optogenetics, nucleus accumbens, amygdala, ventral tegmental area. 
Nicotine is the principal addictive component that drives continued tobacco use. The initiation of addiction involves the mesocorticolimbic dopamine (DA) system, which contributes to the processing of rewarding stimuli during the overall shaping of successful behaviors (Schultz, 2007). It has been hypothesized that addictive drugs such as nicotine hijack the same mechanisms as reinforcement learning, leading to an overvaluation of the drug reward at the expense of natural rewards. While drug-induced reinforcement learning generally involves an increase in extracellular DA concentration in the nucleus accumbens (NAc), the underlying molecular and cellular mechanisms are drug dependent (Changeux, 2010; Di Chiara and Imperato, 1988; Luscher, 2016). Nicotine, for instance, exerts its reinforcing effects through the direct activation of nicotinic acetylcholine receptors ( $\mathrm{nAChRs),} \mathrm{a} \mathrm{family} \mathrm{of} \mathrm{pentameric} \mathrm{ligand-gated} \mathrm{ion} \mathrm{channels}$ (Changeux et al., 1998), on midbrain DA and GABA neurons, thus increasing the activity of both neuronal populations (Maskos et al., 2005; Morel et al., 2014; Tolu et al., 2013). Cell type-specific optogenetic manipulations have confirmed that DA cell activation is sufficient to drive the transition toward addiction, and established causal links between DA neuron activation and drug-adaptive behaviors (Pascoli et al., 2015). However, such view does not take into account the heterogeneity of midbrain DA neurons and the possibility that different messages can be transmitted in parallel from DA neurons of the ventral tegmental area (VTA). Indeed, VTA DA neurons belong to anatomically distinct circuits, differ in their molecular properties, and show diverse responses to external stimuli (Lammel et al., 2008; Poulin et al., 2018). DA neurons not only transmit signals related to salience and reward, but also to aversive stimuli (Brischoux et al., 2009; de Jong et al., 2019), including the negative effects of nicotine at high doses (Grieder et al., 2019; 2010). However, how DA neurons simultaneously transmit opposing signals in response to the same stimuli remains unclear. Whereas the vast majority of research teams that have examined nicotineevoked responses report a homogenous activation of DA neurons and an increase in DA release in their projection areas (Di Chiara and Imperato, 1988; Grenhoff et al., 1986; Mansvelder and McGehee, 2000; Maskos et al., 2005; Picciotto et al., 1998; Zhao-Shea et al., 2011), other reports suggested that DA neuron responses to nicotine are more heterogeneous than previously thought (Eddine et al., 2015; Mameli-Engvall et al., 2006; Zhao-Shea et al., 2011). Therefore, a key issue is how the multiple effects of nicotine map onto the DA cell diversity, and whether nAChRs or other features can define different neuronal subpopulations that, through their response to nicotine, would influence specific behaviors.

Results

\section{DA neurons in the VTA display opposite responses to acute nicotine injection}

We performed single-cell electrophysiological recordings on anaesthetized mice, using an intravenous (IV) injection of a reinforcing dose of nicotine (30 $\mathrm{\mu g} / \mathrm{kg}$ ) (Morel et al., 2014), to record the responses of VTA DA neurons $(n=245)$. These neurons were first identified during the recording based on their electrophysiological properties (i.e., firing rate and action potential width (Mameli-Engvall et al., 2006; Ungless and Grace, 2012)), and then filled with neurobiotin by the juxtacellular labeling technique (Eddine et al., 2015; Pinault, 1996). All neurons were confirmed as DA neurons post-hoc by immunofluorescence co-labeling with tyrosine hydroxylase (TH) (Figure 1A). Acute nicotine injections induced a significant variation, either positive or negative, of DA neuron firing rates (Figure 1B, left). Among the $245 \mathrm{DA}$ neurons identified, some were activated $(n=155)$ whereas others were inhibited $(n=90)$ by the nicotine injection 
compared to the control saline injection, as previously reported (Eddine et al., 2015). The amplitudes of nicotine-induced activation and inhibition were similar, at about $\pm 35 \%$ from baseline (Figure 1B, right). We first sought to determine whether the opposing responses to nicotine injection between these DA neuron populations were associated with a difference in their spontaneous activity. The spontaneous activity of VTA DA neurons is characterized by their firing rate and the percentage of spikes within a burst (\%SWB) (Mameli-Engvall et al., 2006). Bursts are classically identified as discrete events consisting of a sequence of spikes with (i) burst onset defined by two consecutive spikes within an interval $<80 \mathrm{~ms}$ and (ii) burst termination defined by an inter-spike interval > $160 \mathrm{~ms}$ (Grace and Bunney, 1984a; Ungless and Grace, 2012). We found that DA neurons activated or inhibited by the nicotine injection had similar firing rates $(\Delta=0.3 \mathrm{~Hz}, p=0.054)$, however the inhibited neurons showed more bursting activity than the activated ones $(\Delta=5.1 \%, p=0.04$ ) (Figure $1 C$, left). An analysis of the distribution of burst time intervals also highlighted different profiles in the distribution of interspike intervals depending on the burst length (Figure $1 \mathrm{C}$, right). Other parameters describing cell spontaneous activity (e.g. coefficient of variation, burst length, ...) were analyzed, but none of them revealed a difference between activated and inhibited DA neurons (not shown). We thus observed small differences in bursting activity between activated and inhibited DA neurons, but we were not able to predict their nicotine-evoked responses based on the analysis of their spontaneous activity, either by traditional classification procedures or by machine learning (Figure S1 A - C). We next asked whether these two populations are anatomically segregated. Neurobiotin-filled cell bodies of each recorded neuron were positioned onto slices of the Mouse Brain Atlas (Paxinos and Franklin, 2004) (Figure S1 D) to study their anatomical location. As illustrated by a single atlas plate schematic (bregma - $3.3 \mathrm{~mm}$ ), anatomical coordinates suggested that the inhibited neurons were located more medially within the VTA than the activated neurons, independently of their antero-posterior or dorso-ventral positions (Figure 1D).

\section{The two subpopulations of DA neurons in the VTA, activated or inhibited by nicotine injection, have distinct axonal projections to the nucleus accumbens or the amygdala}

The DA system is heterogeneous, and is increasingly thought about in terms of anatomically and functionally distinct subnetworks (Watabe-Uchida et al., 2012). DA neurons in the VTA have been reported to project to different terminal regions based on their localization along the mediolateral axis (Beier et al., 2019; 2015; Lammel et al., 2008), thus we next investigated whether these two subpopulations belong to anatomically distinct dopamine circuits by probing the nicotine-evoked responses of DA neurons with identified projection sites. To do so, green retrobeads (RB), a retrograde tracer, were injected either in the nucleus accumbens (NAc: shell and core) or in the amygdala nuclei (Amg: basolateral and central amygdala) (Figure S2.1). Two weeks later, spontaneous and nicotineevoked activity of VTA DA neurons were recorded in vivo in anesthetized mice, and then all neurons were labeled with neurobiotin. Triple labeling immunofluorescence allowed us to confirm post-hoc the DA nature $(\mathrm{TH}+)$, projection site (RB+ / RB-), and position $(\mathrm{NB}+$ ) of the recorded neurons (Figure $2 \mathrm{~A})$ ). We first noted that, in line with previous reports (Lammel et al., 2008), Amg-projecting DA neurons are located more medially in the VTA than NAc-projecting DA neurons (Figure S2.1). We identified 32 nicotineactivated and 17 nicotine-inhibited cells in mice with RB injected in the NAc, and 26 nicotine-activated and 26 nicotine-inhibited cells in mice with RB injected in the Amg (Figure 2B, E). For mice with RB injection in the NAc, $93 \%(28 / 30)$ of NAc-projecting VTA DA neurons ( $\mathrm{RB}+, \mathrm{TH}+)$ were activated by a nicotine injection, while $7 \%(2 / 30)$ of neurons were inhibited. In contrast, in these mice $79 \%(15 / 19)$ of DA 
111 neurons without evidence for projection to the NAc (RB-, $\mathrm{TH}+$ ) were inhibited by a nicotine injection, and 112 only $21 \%$ (4/19) of RB- neurons were activated (Figure $2 \mathrm{C}$ ). For mice with RB injection in the Amg, $86 \%$ 113 (19/22) of Amg-projecting VTA DA neurons (RB+, TH+) were inhibited by a nicotine injection, while 14\% 114 (3/22) were activated. Whereas, in these mice, DA neurons without evidence for projection to the Amg $115(\mathrm{RB}-, \mathrm{TH}+)$ were mainly activated by a nicotine injection (77\%, 23/30), with $23 \%(7 / 30)$ of neurons inhibited 116 (Figure 2E, F). Overall, these results indicate that the majority of VTA DA neurons activated by nicotine 117 injection project to the NAc, whereas the majority of VTA DA neurons inhibited by nicotine project instead 118 to the Amg. We next took advantage of this anatomical distinction to analyze their respective 119 electrophysiological properties in ex vivo patch-clamp recordings on coronal VTA slices with NAc120 projecting DA cells or Amg-projecting DA cells labeled with RB (Figure S2.2). Amg-projecting DA neurons 121 demonstrated higher excitability (Figure S2.2B) than NAc-projecting DA neurons, but no difference in 122 nicotine-evoked currents was found between these two populations (Figure S2.2D). These results indicate 123 that these two VTA DA cell populations have different membrane properties, but do not markedly differ in 124 the expression or function of $\mathrm{nAChRs}$.

\section{The anxiogenic effect of nicotine injection involves $\beta 2$ subunit-containing $n A C h R s$ in the VTA}

We next asked whether these two distinct dopamine sub-circuits are associated with different behavioral outcomes after an acute injection of nicotine. Nicotine is known to have rewarding properties, through the activation of VTA DA neurons (Durand-de Cuttoli et al., 2018; Maskos et al., 2005; Tolu et al., 2013). Nicotine can also induce negative outcomes such as anxiety-like behaviors and stress-induced depressive-like states (Kutlu and Gould, 2015; Morel et al., 2017; Picciotto and Mineur, 2013). However, whether these negative effects are mediated by VTA DA neurons is unknown. We thus asked whether the anxiogenic effect of nicotine is mediated by VTA DA neurons, by analyzing mouse behavior in an elevated-O-maze (EOM). First, we found that mice injected with nicotine (intra-peritoneal, IP, $0.5 \mathrm{mg} / \mathrm{kg}$ ) spent less time in the open arms of the EOM when compared to mice injected with saline, indicating that nicotine triggers anxiety-like behavior in mice (Figure 3A and Figure S3.1 for individual data). This anxietylike phenotype was not related to a noticeable nicotine effect on locomotor activity (Figure S3.1). To provide evidence for a specific role of VTA neuron response to nicotine in this anxiogenic effect, we then locally infused nicotine into the VTA by a fixed implanted cannula, and found that, just as observed with the IP nicotine injection, these mice spent less time in the open arms of the EOM than those injected with saline (Figure 3B and Figure S3.1 for individual data). Local nicotine infusion in the VTA was therefore sufficient to trigger such anxiogenic effect. Finally, as nicotine-evoked responses have been shown to be mainly mediated by $\beta 2$-containing $n A C h R s ~\left(\beta 2^{*} n A C h R s\right)$ expressed on the soma of both DA and GABA neurons of the VTA (Tolu et al., 2013), we next assessed the role of $\beta 2^{*} n A C h R s$ within the VTA in mediating the anxiogenic effects of nicotine by using knock-out mice deleted for the $n A C h R ~ \beta 2$ subunit ( $\beta 2^{--}$mice). Electrophysiological recordings of VTA DA neurons in $\beta 2^{-/-}$mice demonstrated neither excitatory nor inhibitory responses to nicotine injection. In contrast, lentiviral re-expression of the $\beta 2$ subunit restricted to the VTA of $\beta 2^{-- \text {- mice }}\left(\beta 2^{--\mathrm{Vec}}\right.$ mice), restored both excitatory and inhibitory responses

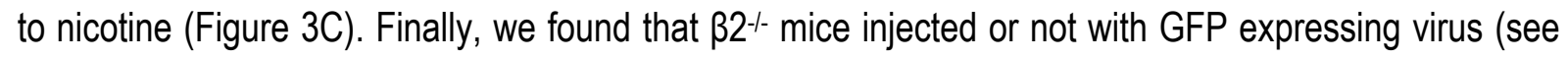

Figure S3F) were impervious to the anxiogenic effect of nicotine in the EOM, as nicotine did not reduce 150 the time spent by $\beta 2^{--}$mice in the open arms, but that this effect could be restored by re-expression of 151 the $\beta 2$ subunit within the VTA ( $\beta 2^{--V}$ Vec, Figure $\left.3 \mathrm{D}\right)$. Together, these results indicate that both the activation or inhibition of VTA DA neurons by nicotine, as well as the anxiogenic effects of nicotine in the 
153 EOM, require signaling through $\beta 2^{*} n A C h R s$ in the VTA. However, the present data do not allow us to

154 conclude whether the anxiogenic effect of nicotine requires the activation of VTA DA neurons, their

155 inhibition, or both types of response.

156

\section{The two projection pathways of DA neurons of the VTA underlie opposite functions in reinforcement and anxiety}

To dissociate whether nicotine-evoked activation or inhibition of VTA DA neurons is necessary for the behavioral effects of nicotine, one would ideally need to isolate these responses in DA neurons, as well as in VTA GABA neurons, which also express nAChRs (Grieder et al., 2019; Tolu et al., 2013). Because activation and inhibition by nicotine are concomitant and not easily isolable from one another, and because the responses of VTA DA and GABA neurons to nicotine are also tightly linked (Tolu et al., 2013), we decided to mimic each response separately with an optogenetic approach. We used a calcium translocating channelrhodopsin (CatCh (Kleinlogel et al., 2011) for activation and a red-shifted cruxhalorhodopsin (Jaws (Chuong et al., 2014)) for inhibition (Figure S4.1). Dopamine transporter (DAT)Cre mice, in which Cre recombinase expression is restricted to DA neurons without disrupting endogenous DAT expression (Turiault et al., 2007; Zhuang et al., 2005), were bilaterally injected in the VTA with CatCh (AAV5-flox-EF1a-hCatCh-YFP), Jaws (pXR5-CAG-flex-Jaws-eGFP), or a control enhanced yellow fluorescent protein (eYFP) with no opsin (AAV5-flox-EF1a-YFP, Figure S4.2A-B), and optical fibers were implanted either in the Basolateral Amg (BLA, Figure S4.2C) or in the NAc lateral shell (NAcLSh, Figure S4.2D) of these mice to restrict the effects of optogenetic stimulation to DA terminals within these regions.

We first examined the effects of these manipulations in the EOM test. Light-evoked inhibition of DA neuron terminals in the BLA reduced the percentage of time spent by Jaws-expressing mice in the open arms of the EOM, while no effect of the light stimulation was detected in control mice expressing only eYFP (Figure $4 \mathrm{~A}$ and Figure S4.3A for individual data). Light-evoked activation of DA terminals in the BLA showed the opposite effect, increasing the percentage of time spent by CatCh-expressing mice in the open arms of the EOM compared to mice expressing eYFP (Figure 4B and Figure S4.3B for individual data). In contrast, light-evoked activation of DA neuron terminals in the NAcLSh of CatCh-expressing mice had no effect on the time spent in the open arms of the EOM compared to control mice expressing eYFP (Figure 4C and Figure $\mathrm{S} 4.3 \mathrm{C}$ for individual data), and there was no noticeable effect of the light-stimulation on locomotor activity in any of these groups of DAT-Cre mice (Figure S4.3D-F). To determine whether the anxiogenic effect observed during inhibition of DA neuron terminals in the BLA was specific to the BLA nuclei, we compared groups of WT mice injected with either Jaws or GFP in the VTA and implanted with bilateral optical-fibers either in the BLA or in the central amygdala (CeA), which both receive DA input (Figure S4.3). We found that inhibiting VTA neuron terminals decreased the percentage of time spent in the open arms of the EOM when optical fibers were implanted in the BLA, but not when they were implanted in the CeA (Figure S4.4). Finally, to directly link nicotine-evoked inhibition of DA neurons to its anxiogenic effect, we reasoned that an optogenetic activation of the BLA-projecting terminals of VTA DA neurons would counteract the nicotine-evoked inhibition of this pathway, and should therefore abolish the anxiogenic effect of the drug. Thus, DAT-Cre mice expressing CatCh in the VTA were injected with IP nicotine one minute before the EOM test, and received light stimulation in the BLA throughout the 9-minute test. Lightevoked activation of BLA DA terminals during the EOM abolished the anxiogenic effect of nicotine injection 
194 in CatCh expressing mice when compared to eYFP expressing controls, which decreased the percentage

195 of their time spent in the open arms after IP nicotine injection (Figure 4D).

196 We next investigated the effects of activating and inhibiting these two pathways on reinforcement using 197 an online place preference paradigm (OPP). Activation of DA neuron terminals in the NAcLSh increased 198 the preference score (percentage of time in ON - percentage of time in OFF) in mice expressing CatCh, 199 indicating a rewarding effect of stimulating the VTA-NAcLSh pathway (Figure 4E). Light-evoked inhibition 200 of DA neuron terminals in the BLA, however, reduced the preference score for the compartment where 201 animals were photo-stimulated, and activation of these terminals showed no effect (Figure 4E).

202 Together, these results argue for a functional dissociation of these two pathways: inhibition of Amg203 projecting DA neurons evokes anxiogenic effects, while activation of NAc-projecting DA neurons mediates 204 reward but has no effect on anxiety-like behavior. Furthermore, we show that the VTA-BLA DA pathway 205 is the substrate for the anxiogenic effect of nicotine.

\section{Discussion}

207 The VTA had long been perceived as a structure that broadly disseminates DA in the brain, with the 208 different time courses of DA activity providing a phenomenological account for the functional involvement 209 of DA neurons in different behavioral and neural processes (Schultz 2007). This temporal account of DA 210 neuron function was gradually replaced or extended by the notion that the DA system, in particular the 211 VTA, is divided into subpopulations of DA neurons, each associated with distinct appetitive, aversive, or 212 attentional behaviors (Lammel et al., 2012). However, we are only beginning to appreciate how the 213 functional activation/inactivation dynamics within these subpopulations impact behavioral and neural 214 processes. Here, we show that activation and inhibition of VTA DA neurons appear concurrently as a 215 consequence of nicotine injection. Furthermore, our results demonstrate that these activation/inhibition 216 processes correspond to two anatomically and functionally distinct circuits, which mediate contrasting 217 behavioral effects.

218 VTA DA neurons are known to be heterogeneous in their axonal projections, electrophysiological 219 properties, and in several molecular features. They show for example striking differences in their 220 expression of hyperpolarization-activated cyclic nucleotide-gated cation channels (HCN), the dopamine 221 transporter (DAT), the dopamine receptor D2R, or vesicular glutamate transporters (VGLUTs) (Lammel 222 et al., 2008; Margolis et al., 2008; Morales and Margolis, 2017). However, the role and the functional 223 consequences of VTA DA neuron heterogeneity in behavior remain poorly understood. Here, we have 224 demonstrated that nicotine evokes opposite responses in two distinct subpopulations of VTA DA neurons, 225 those that project their axons to the NAc are activated by nicotine, while those that project to the Amg are 226 inhibited. In addition to their functional and anatomical segregation, we found that these subpopulations 227 display different bursting activities in vivo, and different excitabilities in vitro. However, they cannot be 228 distinguished solely on the basis of their spontaneous firing pattern in anesthetized mice. Are there 229 specific differences between these two neuronal populations, beside their projection sites, that would 230 underlie their opposing responses to nicotine injection? NAc-projecting DA neurons exhibit smaller $I_{h}$ 231 currents than BLA-projecting DA neurons, but have similar input resistances and capacitances (Ford et 232 al., 2006), and similar expressions of DAT, D2R and TH (Su et al., 2019). We have previously reported 233 that activated and inhibited DA cells react similarly to D2R agonist or antagonist injection in vivo, which is 
also consistent with similar D2R expression levels in the two neuronal populations (Eddine et al., 2015). Finally, there is no clear variation in nicotine-evoked currents in Amg or NAc-projecting DA cells, suggesting that nAChR expression does not differ markedly between these populations. Overall, we did not find striking evidence of intrinsic differences between NAc-projecting or Amg-projecting VTA DA neurons that could explain their differences in the nicotine-evoked responses. While differences may exist, it is also possible that the emergence of either nicotine-evoked activation or inhibition of these neurons arises from network dynamic. Within the VTA, nicotine directly activates both DA and GABA neurons, as they each express nAChRs (Klink et al., 2001; Tolu et al., 2013). In particular, $\beta 2{ }^{*} n A C h R s$ of the VTA are key mediators of the positive reinforcing effects of nicotine, as previously shown by reexpressing $\beta 2$ locally in the VTA of $\beta 2^{-/-}$mice (Maskos et al., 2005; Tolu et al., 2013), or by rendering $\beta 2^{*} n A C h R s$ insensitive to nicotine using light (Durand-de Cuttoli et al., 2018). Here, we show that $\beta 2^{*}$ nAChRs of VTA neurons are also required to evoke the inhibition of the VTA-Amg DA neuron subpopulation after systemic nicotine injection. Therefore, nicotine acting through $\beta 2^{*} n A C h R s$ activates VTA GABAergic interneurons and DA neurons that project to the NAc, while simultaneously inhibiting DA neurons projecting to the Amg. Several independent mechanisms may explain the inhibitory effect of nicotine on this subpopulation. These include (1) the activation of GABAergic interneurons that would, in turn, inhibit specifically this VTA DA neuron subpopulation; (2) inhibition through local DA release (Eddine et al., 2015), even though no difference in D2R-mediated inhibitory postsynaptic currents or in DA reuptake between NAc-projecting and BLA-projecting DA neurons has been reported (Ford et al., 2006); or (3) feedback inhibition. Indeed, subpopulations of DA neurons are embedded within distinct inhibitory networks resulting in specific feedback loops between VTA and NAc subregions (de Jong et al., 2019). NAc-projecting DA neurons could therefore inhibit DA neurons projecting to the Amg through an as yet unknown striatal relay.

Nicotine is highly reinforcing, but also generates aversive and anxiogenic effects at various doses (Balerio et al., 2006; Kutlu and Gould, 2015; Picciotto and Mineur, 2013; Wolfman et al., 2018). Thus, depending on the context, the exact same dose of nicotine can trigger anxiety and/or reinforcement. Aversion for high doses of nicotine and anxiety associated with nicotine withdrawal have been attributed to nicotinic and glutamatergic signaling in the habenulo-interpedoncular axis (Fowler et al., 2011; Frahm et al., 2011; Molas et al., 2017; Zhao-Shea et al., 2013). There is also evidence that nAChRs of neurons located in the Amg modulate depressive-like states (Mineur et al., 2016). However, a role for DA in aversion has also been proposed. D1R and D2R antagonists prevent conditioned-place aversion induced by an acute high-dose nicotine injection (Grieder et al., 2012), and $\beta 2^{*}$ nAChRs were shown to be necessary for both the aversive and rewarding effects of nicotine using a subunit re-expression strategy in VTA DA and GABAergic neurons of $\beta 2^{-/-}$mice (Grieder et al., 2019). However, the mechanism underlying these opposite effects of the drug has not yet been established. Here, we show that activation of $\beta 2^{*} n A C h R s$ of VTA neurons is necessary for nicotine to inhibit Amg-projecting DA neurons and induce anxiety-like behavior. This indicates that DA signaling is critically involved in the acute anxiogenic effect of nicotine and could also mediate aversion to nicotine. Moreover, injections of nicotine at the doses used in this study are known to be rewarding in different paradigms in mice, which has been attributed to VTA DA neuron activation (Durand-de Cuttoli et al., 2018; Maskos et al., 2005; Tolu et al., 2013). Overall, our study

274 shows that the same intake of nicotine can induce a rewarding effect by activating the VTA-NAc dopamine 275 pathway, and simultaneously signal a negative emotional state by inhibiting the VTA-Amg dopamine 276 pathway. 
277 Our findings emphasize the complex role of the DA system in not only positive but also negative 278 motivational processes, and promote a more nuanced view of the effects of reinforcing doses of nicotine 279 on VTA DA neurons. Opposing responses of DA neurons to drug exposure have also been observed with 280 cocaine (Mejias-Aponte et al., 2015), ethanol (Doyon et al., 2013), and morphine (Margolis et al., 2014). 281 Notably, the inhibition induced by opioids differs in BLA-projecting and NAc-projecting VTA DA neurons 282 (Ford et al., 2006), suggesting that the behavioral effects of opioid drugs could also result from a specific 283 pattern of inhibition in these two pathways. Since our results demonstrate that both rewarding and 284 anxiogenic messages occur simultaneously upon nicotine exposure, and are conveyed by distinct 285 subpopulations of VTA DA neurons, the question then arises as to how the concurrent engagement of 286 two circuits with opposing messages could compete to produce nicotine reinforcement, and whether an 287 imbalance between the two would lead to addiction. Indeed, this question may prove critical when it comes 288 to medical strategies aimed at smoking cessation. While the optogenetic strategies used in this study are 289 well suited to mimic the individual effects of a drug that also produces strong and synchronized neuronal 290 activity, the translational value of these effects is perhaps not to be sought in the specific activation or 291 inhibition of a given neuronal pathway, but rather in the functional imbalance that this creates between 292 the target structures of VTA neurons. Nevertheless, a detailed understanding of the multiple pathways 293 engaged in nicotine-evoked responses and of their respective behavioral contributions can still help us 294 understand the mechanisms leading to nicotine addiction. In this respect, the activation and inhibition 295 processes which appear in VTA DA neurons as a consequence of systemic nicotine injection call for 296 further mechanistic studies, but we show that they correspond to discrete neuronal circuits and that they 297 mediate distinct behavioral effects, both of which are relevant to the understanding of addiction. 


\section{Acknowledgements:}

299 We thank Lauren Reynolds for critical reading of the manuscript. We are grateful to France Lam and the 300 imaging platform facility (IBPS), the animal facilities (IBPS), Victor Gorgievski for behavioral data 301 acquisition. We are grateful to Mélissa Desrosiers, Camille Robert and Paris Vision Institute AAV 302 production facility for viral production and purification.

303 This work was supported by the Centre National de la Recherche Scientifique CNRS UMR 8246, INSERM 304 U1130, the Foundation for Medical Research (FRM, Equipe FRM DEQ2013326488 to P.F), FRM 305 FDT201904008060 (to SM), the French National Cancer Institute Grant TABAC-16-022 et TABAC-19306020 (to P.F.), French state funds managed by the ANR (ANR-16 Nicostress to PF, ANR-19 Vampire to 307 FM), The LabEx Bio-Psy (to P.F and Doctoral Fellowship to CNG). PF and UM are members of LabEx 308 Bio-Psy.

\section{Author contributions:}

310 C.N., F.M. and P.F. designed the study. C.N., F.M. and P.F. analyzed the data. C.N. and F.M. performed 311 in vivo electrophysiological recordings. S.T. and S.V. contributed to in vivo electrophysiological 312 recordings. S.M. performed ex vivo patch-clamp recordings and data analyses. T.L.B. contributed to in 313 vivo electrophysiological data analyses. C.N. performed injections, fiber and cannula implantations. C.N. 314 and I.C performed behavioral experiments. S.M. and B.H. contributed to behavioral experiments. R.D.C 315 and A.M contributed to optogenetic experiments. C.N and S.M. performed immunostaining experiments. 316 D.D., S.P. and U.M. provided viruses. U.M. provided ACNB2 KO mice. C.N., J.P.H., A.M., F.M. and P.F. 317 wrote the manuscript.

318 Declaration of interests: Authors declare no competing financial interests.

319 Data and materials availability:

320 All the data are available from the corresponding authors upon request, including the $\mathrm{R}$ code routines. 


\section{Figure Legends}

323 Figure 1: Nicotine evoked opposite responses in DA neurons of the VTA

324 (A) Intravenous (IV) injections of nicotine (Nic, $30 \mu \mathrm{g} / \mathrm{kg}$ ) induce activation and inhibition of distinct VTA 325 DA cells. Post-recording identification of neurobiotin (NB)-labeled VTA DA neurons by 326 immunofluorescence ( $\mathrm{TH}=$ tyrosine hydroxylase, $\mathrm{NB}=$ streptavidin-AMCA against neurobiotin). (B) Left: 327 Cumulative distribution of responses after IV injection of either saline (Sal, grey, $n=233$ ) or nicotine (Nic, 328 black, $n=245$ ) (Kolmogorov-Smirnov test $\left.{ }^{* * *} p<0.001\right)$. Center. Time course for the average change in 329 firing frequency upon nicotine injection for activated (red, $n=155$ ) and inhibited (blue, $n=90$ ) VTA DA 330 neurons. Right: Average amplitude of the nicotine response for activated (red, mean $=33.75 \pm 52 \%$ ) and 331 inhibited DA neurons (blue, mean $=-35.42 \pm-2 \%$ ), compared to saline injection (grey, $n=147$ and 86, 332 respectively) (Wilcoxon test ${ }^{* * *} p<0.001$ ). (C) Analysis of the spontaneous activity of NB-labeled DA 333 neurons that were either activated (red) or inhibited (blue) by the nicotine injection. Left: The two 334 subpopulations of DA neurons did not display any significant difference in their firing rates (Wilcoxon test $335 p=0.054)$. However, neurons that were inhibited by nicotine displayed a basal percentage of spikes336 within-burst (\%SWB) higher than activated ones (Wilcoxon test $\left.{ }^{*} p=0.04\right)$. Right: Interval between SWB 337 (in $\mathrm{ms}$ ) as a function of the length of the burst (from two to six action potentials). D) Localization of NB338 labeled, nicotine-activated and -inhibited DA neurons, positioned on the Paxinos atlas at bregma - 3.3 $339 \mathrm{~mm}$. The subpopulation of nicotine-inhibited neurons had a more medial distribution in the VTA than the 340 nicotine-activated subpopulation (Wilcoxon test ${ }^{* *} p<0.001$ ), but neither antero-posterior (Wilcoxon test $341 p=0.4$ ) nor dorso-ventral (Wilcoxon test $p=0.5$ ) differences in their distribution were observed. 


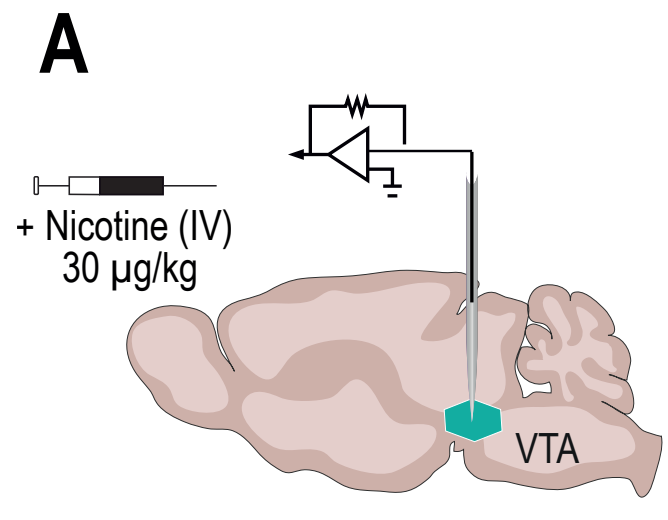

B

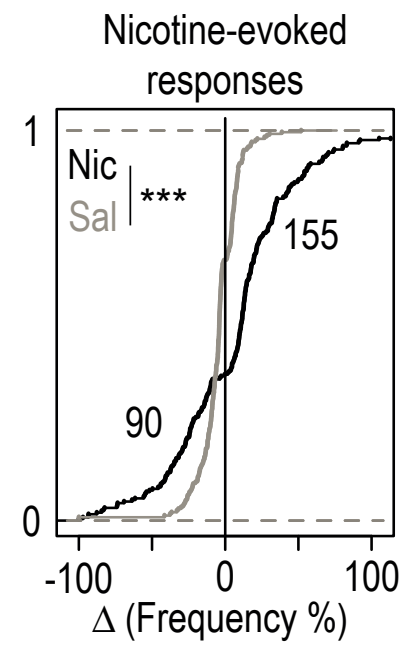

Nic $30 \mu g / k g$, IV

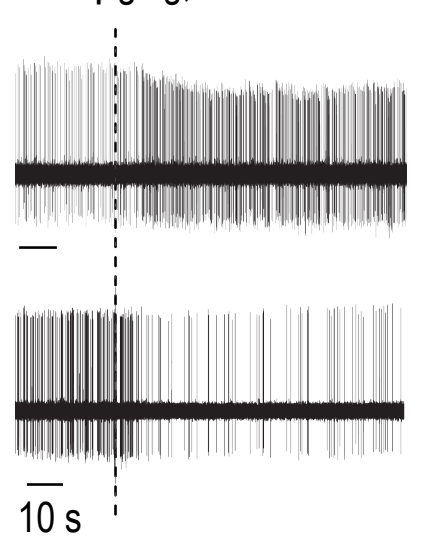

$10 \mu \mathrm{m}$
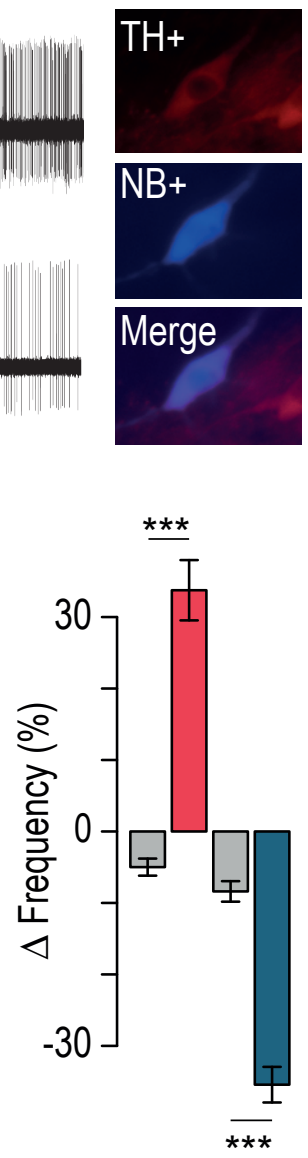

C

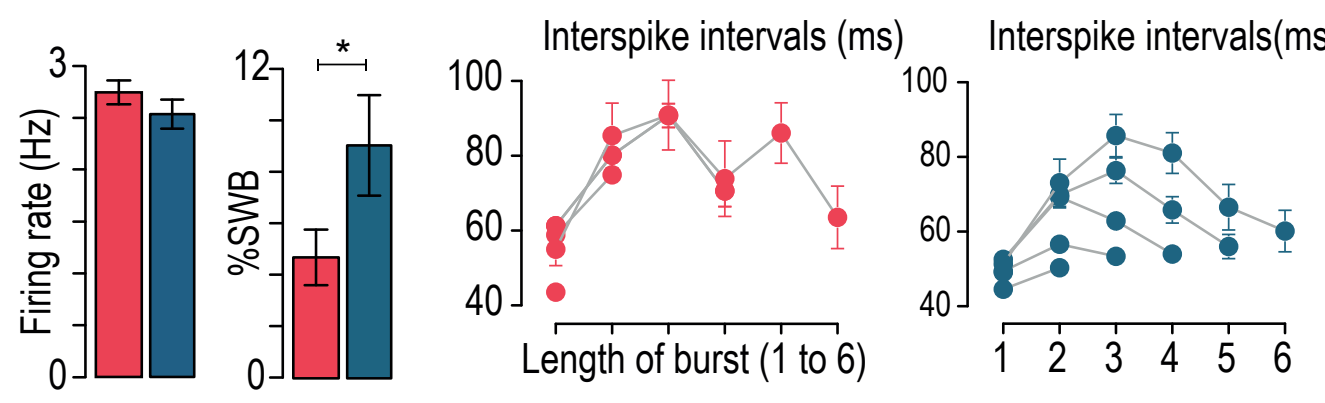

D

$35 \quad 0$

Bregma - $3.3 \mathrm{~mm}$
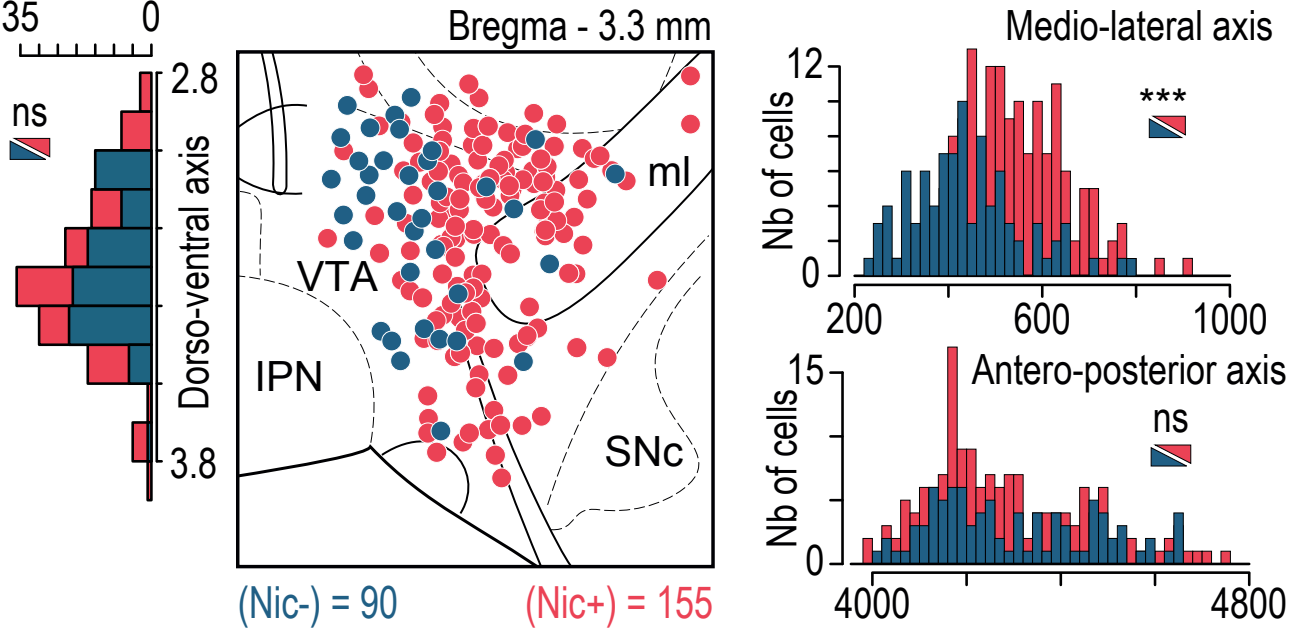

Figure 1 
342 Figure 2: Nicotine evoked opposite responses in VTA DA neurons that project to the NAc or to the

\section{Amg}

344 (A) Retrobeads (RB) were injected in the NAc, and three weeks later VTA DA neuron responses to an IV 345 nicotine injection were recorded in vivo, and neurons were subsequently labeled with neurobiotin. DA 346 neurons that project to the NAc were identified post-hoc by immunofluorescent co-labeling of $\mathrm{TH}, \mathrm{NB}$ and 347 RB. (B) Localization of NB-labeled DA neurons $(\mathrm{NB}+\mathrm{TH}+, \mathrm{n}=49)$ following RB injection into the NAc. $348 \mathrm{RB}+$ and RB- neurons are represented by filled and empty circles, respectively. Red and blue colors 349 denote nicotine-activated neurons (Nic+) and -inhibited neurons (Nic-), respectively. (RB+ Nic+, $n=28$; 350 RB+ Nic-, $n=2$; RB- Nic+, $n=4$; RB- Nic-, $n=15$ ). (C) Top: Percentage and number of Nic+ (red) and 351 Nic- (blue) cells among NAc-projecting DA neurons (RB+). Mean change in firing frequency of NAc352 projecting DA neurons in response to an IV injection of nicotine (red) or saline (black). Bottom: Percentage 353 and number of Nic+ (red) and Nic- (blue) cells in non RB-labeled neurons (RB-). Mean change in firing 354 frequency of RB- DA neurons in response to an IV injection of nicotine (red) or saline (black). (D) In 355 separate experiments, RBs were injected in the Amg, and two weeks later VTA DA neuron responses to 356 an IV Nic injection were recorded in vivo, and neurons were subsequently labeled with neurobiotin. DA 357 neurons that project to the Amg were identified post-hoc by immunofluorescent co-labeling of TH, NB and 358 RB. (E) Localization of NB-labeled DA neurons $(\mathrm{NB}+\mathrm{TH}+, \mathrm{n}=52)$ following RB injection into the Amg. 359 (RB+ Nic+, $n=3 ;$ RB+ Nic-, $n=19 ;$ RB- Nic+, $n=23 ;$ RB- Nic-, $n=7)$. (F) Top: Percentage and number 360 of Nic- (blue) and Nic+ (red) among Amg-projecting DA neurons (RB+) or in RB- DA neurons of the VTA 361 in mice injected in the Amg. Mean change in firing frequency of RB+ DA neurons in response to an IV 362 injection of nicotine (red) or saline (black). Bottom: Percentage and number of Nic- (blue) and Nic+ (red) 363 cells in non RB-labeled neurons (RB-). Mean change in firing frequency of RB- DA neurons in response 364 to an IV injection of nicotine (red) or saline (black). 
A

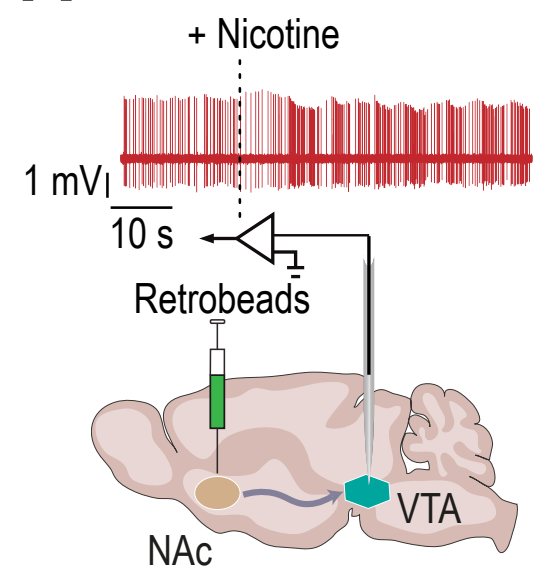

D

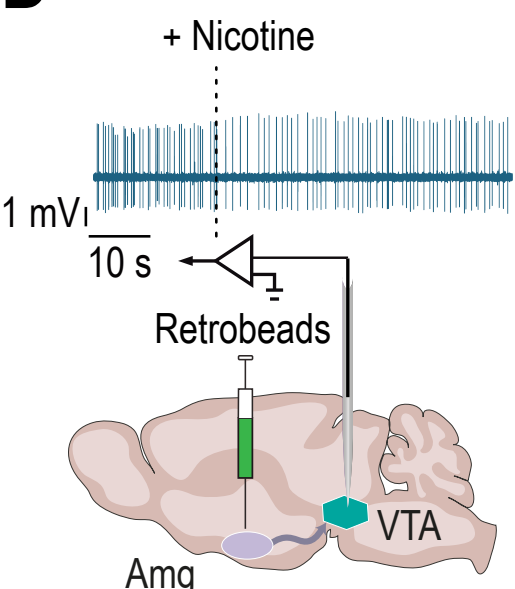

B

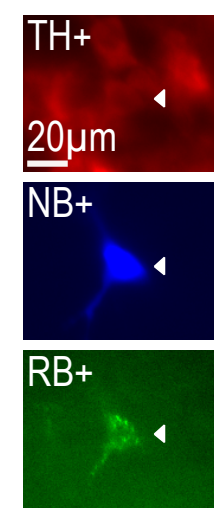

NAc injection Bregma $-3.3 \mathrm{~mm}$

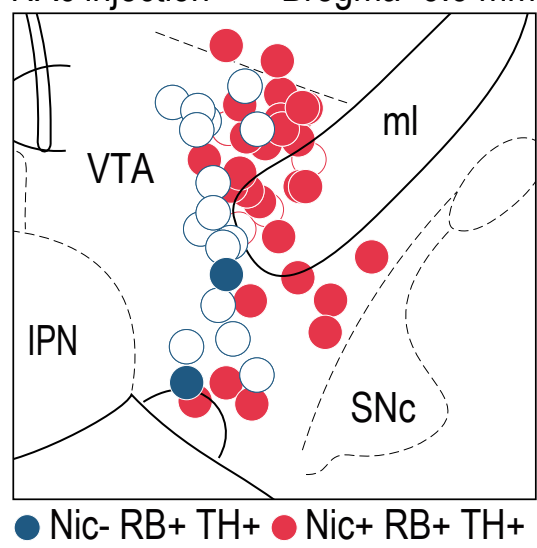

Nic- $\mathrm{RB}-\mathrm{TH}+\mathrm{Nic}+\mathrm{RB}-\mathrm{TH}+$

E

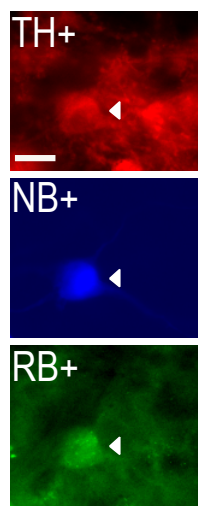

Amg injection Bregma $-3.3 \mathrm{~mm}$

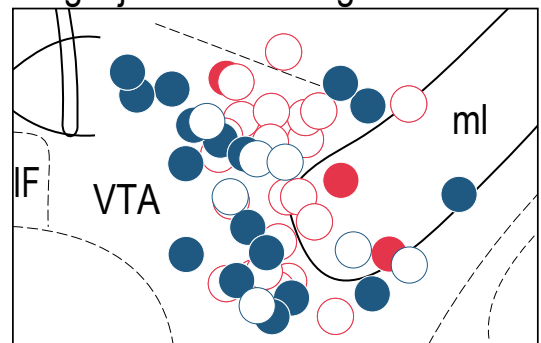

IPN

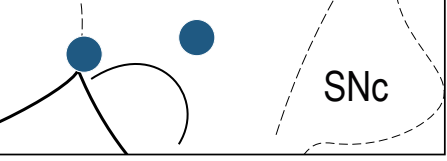

C

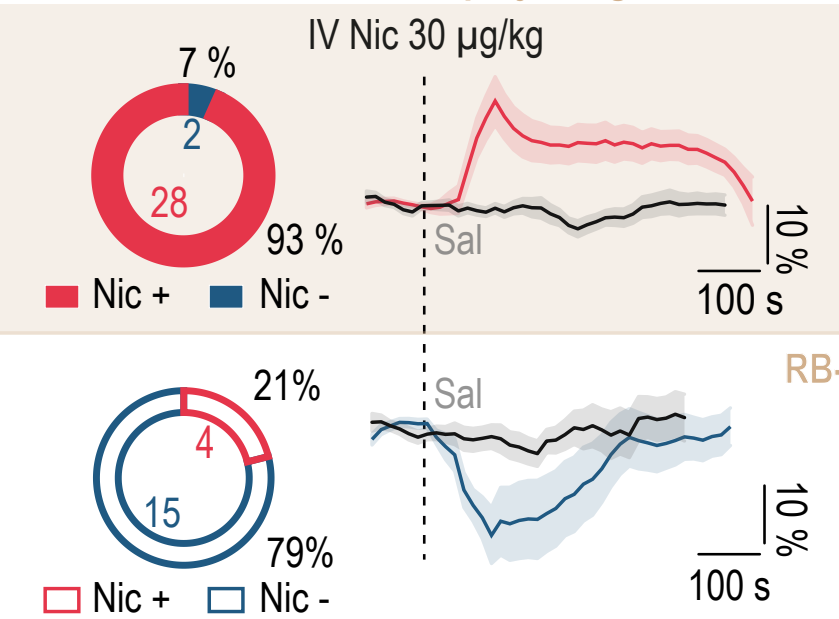

F

- Nic- $\mathrm{RB}+\mathrm{TH}+\mathrm{Nic}+\mathrm{RB}+\mathrm{TH}+$

Nic- RB- TH+ ONic+ RB-TH+
Amg-projecting neurons RB+ IV Nic $30 \mu \mathrm{g} / \mathrm{kg}$

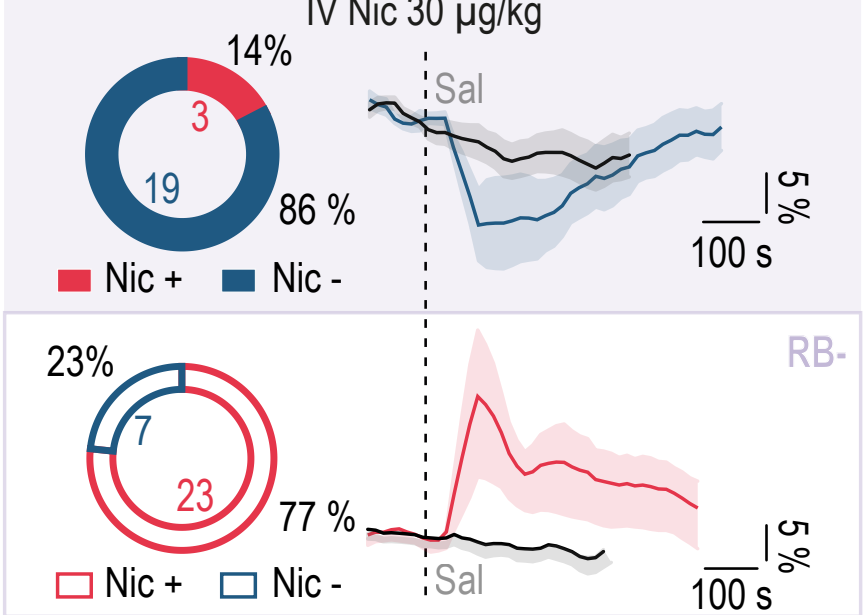

Figure 2 
Figure 3: $\beta 2$ subunit-containing nAChRs mediated both VTA DA neuron responses to nicotine injection and nicotine-induced anxiogenic effects.

(A) Nicotine (Nic, $0.5 \mathrm{mg} / \mathrm{kg}$ ) or saline (Sal) were injected intraperitoneally (IP) 1 minute before the 9minute elevated O-maze (EOM) test. In wild-type (WT) mice $(n=21)$, nicotine injection decreased the time spent in the open arms of the EOM compared to the group injected with Sal $(n=23)$ (two-way RM ANOVA main effect of time $F_{(2,84)}=3.84,{ }^{*} p=0.025$, treatment $x$ time interaction $F_{(2,84)}=5.37$, ${ }^{* *} p=$ 0.006; post-hoc Wilcoxon test with Bonferroni corrections ${ }^{*} p$ ( 3 vs 9 minutes) $=0.03, p$ ( 3 vs 6 minutes) $=0.1, p\left(6\right.$ vs $\left.9^{\prime}\right)=0.2$; post-hoc Wilcoxon test Sal vs Nic at 9 minutes $\left.{ }^{* * *} p<0.001\right)$. (B) Mice implanted with intracranial guide cannulas were infused with either Sal or Nic (100 $\mathrm{ng}$ in $100 \mathrm{nl}$ infusion) over 1 minute before the 9-minute EOM test. The Nic-injected group $(n=7)$ spent less time in the open arms over the test duration than the control group $(n=6)$ (two-way RM ANOVA main effect of time $F_{(2,22)}=$ $12.48,{ }^{* * *} p<0.001$, treatment $x$ time interaction $F_{(2,22)}=9.66,{ }^{* * *} p<0.001$; post-hoc Student's t-test with Bonferroni corrections: ${ }^{* * *} p$ ( 3 vs 9 minutes $)<0.001,{ }^{*} p$ ( 3 vs 6 minutes $)=0.03,{ }^{*} p$ (6 vs 9 minutes $)=$ 0.02 ; post-hoc Student's t-test Sal vs Nic at 9 minutes, $p=0.054$ ). (C) Left: Juxtacellular recording traces of VTA DA neurons in mice deleted for the $\beta 2 \mathrm{nAChR}$ subunit $\left(\beta 2^{--}\right)$and in $\beta 2^{--}$mice transduced with the $\beta 2$ subunit-together with GFP- in the VTA ( $\beta 2--V e c)$. Individual and mean responses (expressed as percentage of firing frequency variation) indicate that there were no nicotine-evoked responses in VTA DA neurons of $\beta 2-$ mice $(n=46$ cells from 12 mice), and that both nicotine-evoked activation ( $n=51$ cells from 18 mice, $\Delta^{+}$) and inhibition ( $n=39$ cells from 19 mice, $\Delta^{-}$) of VTA DA neurons were restored in $\beta 2-$ -Vec mice. Right: Immunofluorescence for TH and GFP on $\beta 2--\mathrm{Vec}$ mice. Cumulative distribution of nicotine-evoked response amplitude of VTA DA neurons in $\beta 2^{-1-}$ mice $(n=46$ cells from 12 mice, grey) and $\beta 2-1$ Vec mice $\left(n=90\right.$ cells from 24 mice, black) (Kolmogorov-Smirnov test $\left.{ }^{* *} p=0.008\right)$. Bar plots show the maximum firing variation induced by nicotine (filled bars) and saline (unfilled bars) in the two groups. Nicotine injection did not alter the firing frequency of VTA DA neurons in $\beta 2^{--}$mice, however it induced a significant increase (mean $12.45 \pm 13.37$ ) or decrease (mean $-13.16 \pm 16.31$ ) in the firing frequency of VTA DA neurons in $\beta 2--$ Vec mice compared to saline $\left({ }^{* * *} p<0.001,{ }^{* * *} p<0.001\right)$ or $\beta 2-1-$ mice ${ }^{* * *} p<0.001,{ }^{* *} p=0.005$ ) (Wilcoxon paired test with Bonferroni corrections) (D) IP nicotine injection $(0.5 \mathrm{mg} / \mathrm{kg})$ in the EOM, for a control group $(n=23)$ of $\beta 2^{-1-}$ mice in which some were injected in the VTA with GFP $(n=6 / 23)$ and for $\beta 2--V e c$ mice $(n=18)$. Re-expression of $\beta 2$ subunit in the VTA restored the nicotine-evoked anxiogenic effects in the EOM, which were absent in the $\beta 2-1-G F P$ mice (two-way RM ANOVA main effect of time $F_{(2,78)}=6.87,{ }^{* *} p=0.002$, treatment $x$ time interaction $F_{(2,78)}=3.43,{ }^{*} p=0.04$; post-hoc Student's t-test with Bonferroni corrections: : ${ }^{* *}$ ( 3 vs 9 minutes $)=0.003,{ }^{*} p$ ( 3 vs 6 minutes $)=$ 398 $0.04, p$ ( 6 vs 9 minutes) $=0.2$; post-hoc Student's t-test $\beta 2-$ and $\beta 2-$ GFP vs $\beta 2--V e c$ mice at 9 minutes, $p=0.06)$. 
A
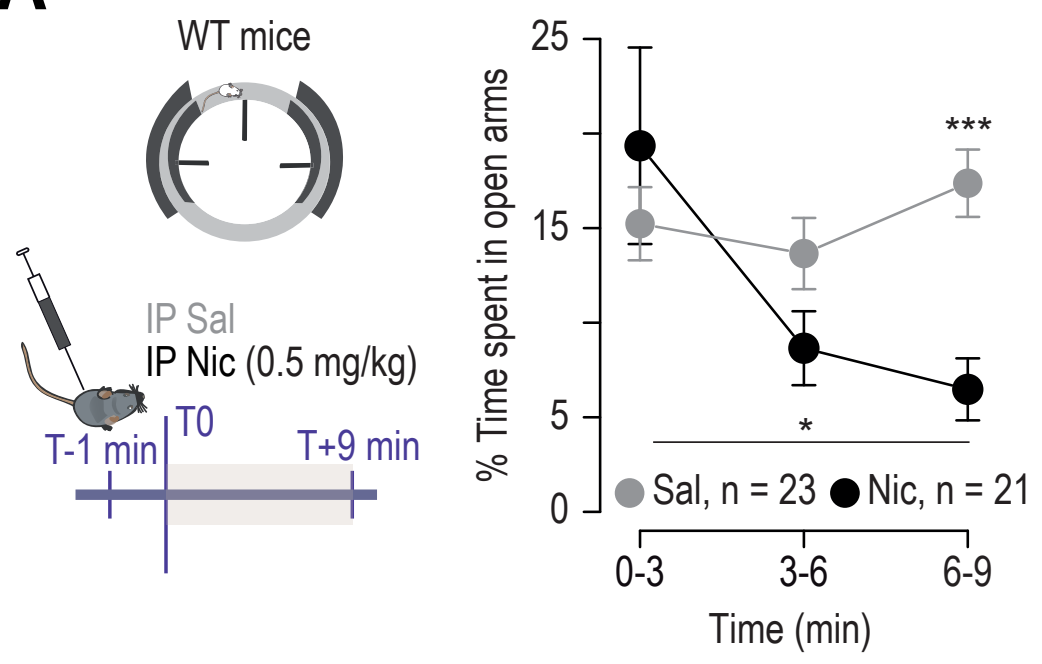

C
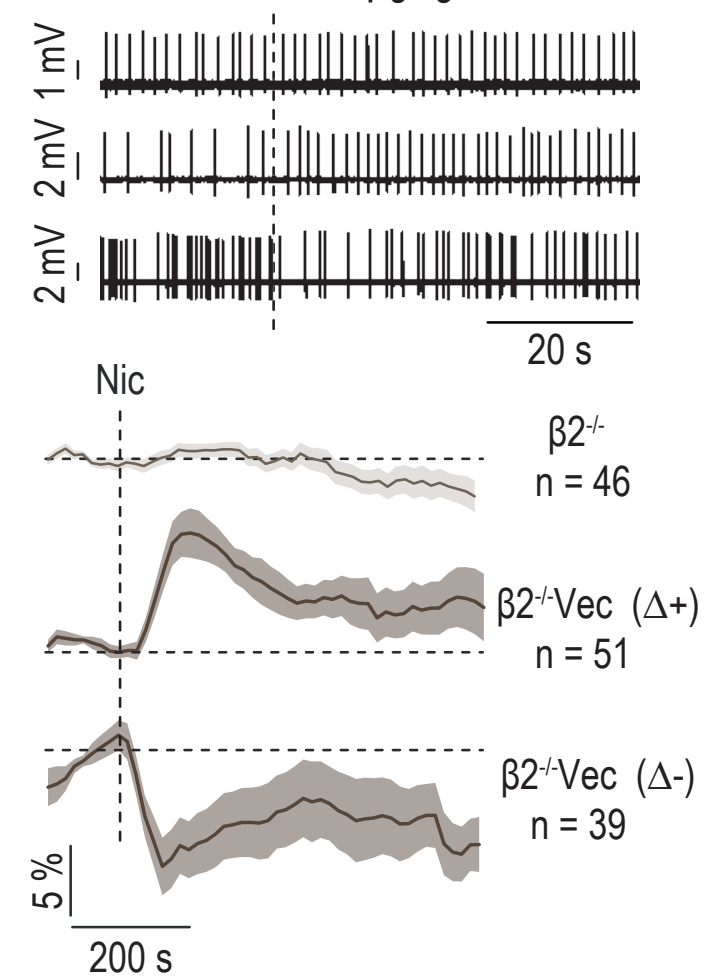

B

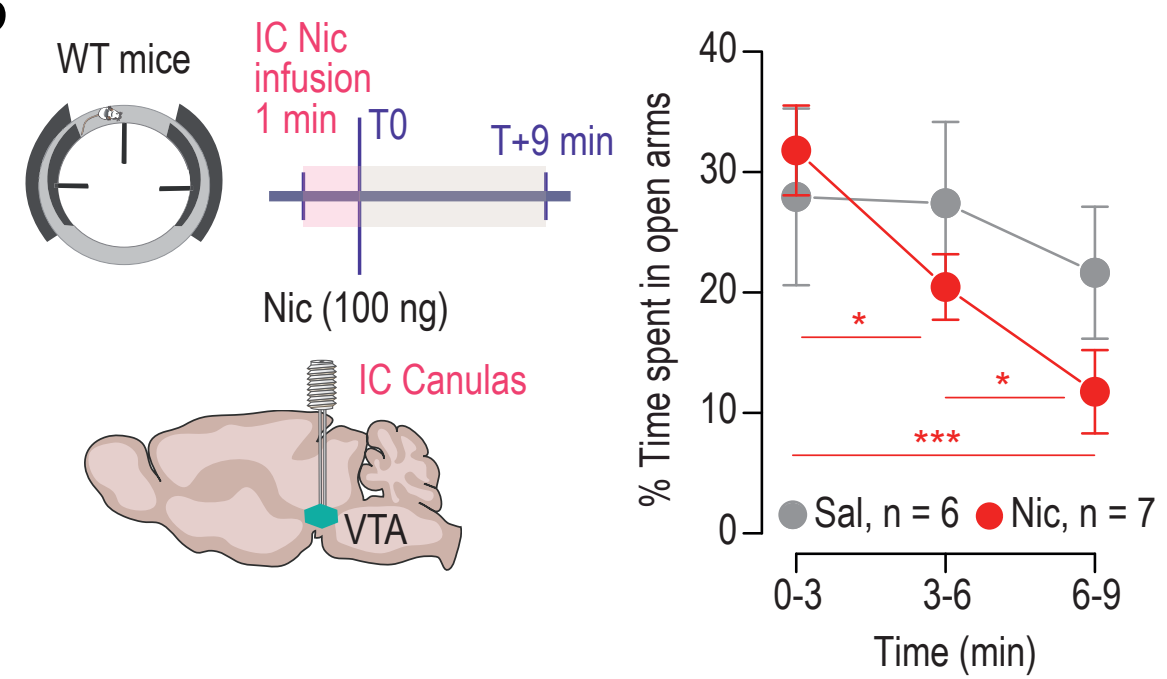

D
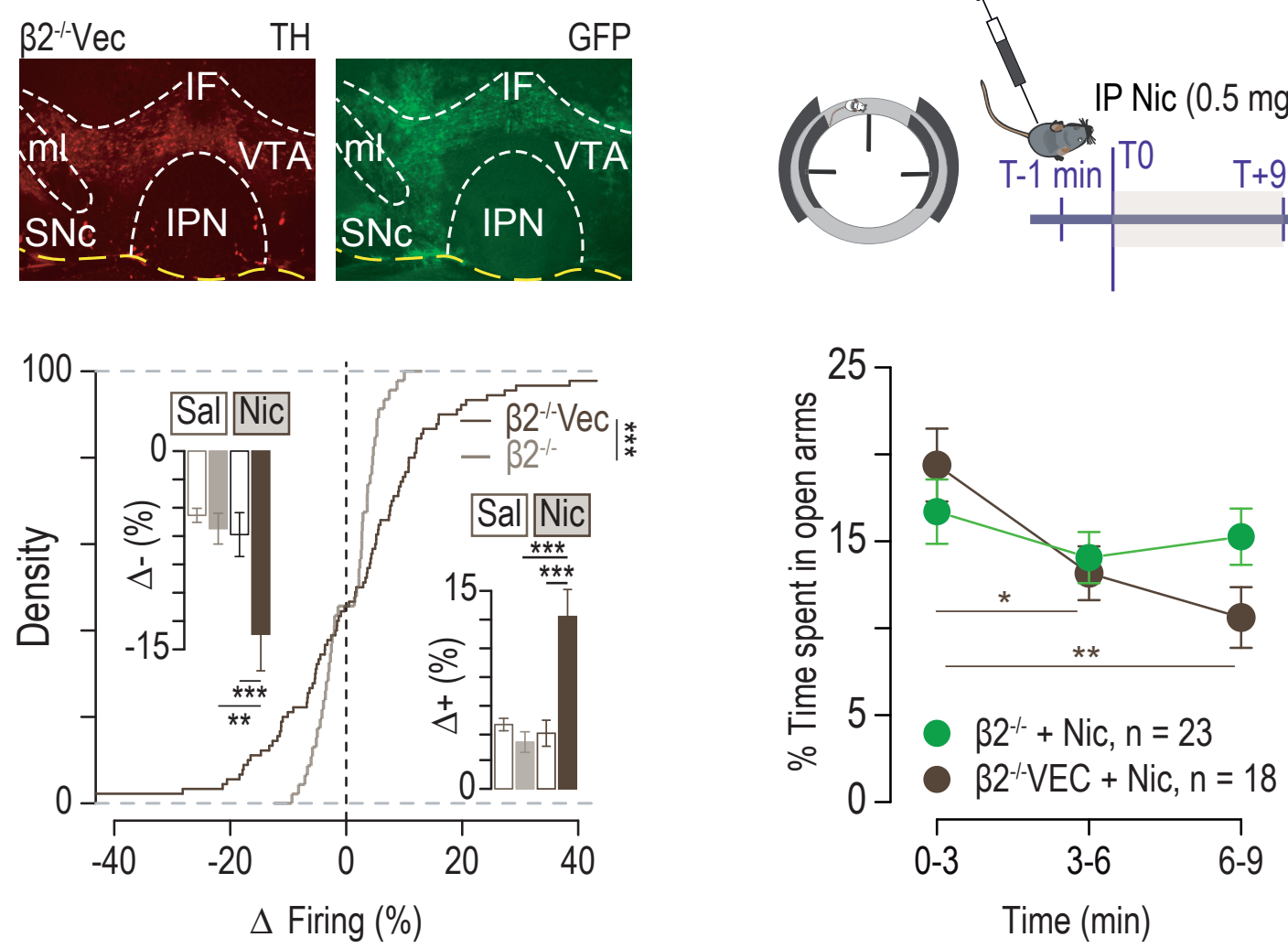

Figure 3 
Figure 4: Selective optogenetic manipulation of VTA-BLA and VTA-NAcLSh pathways highlights the opposite effects of nicotine injection on VTA DA neurons.

(A) Percentage of time spent in the EOM open arms for Jaws (orange, $n=18$ ) and YFP control (green, $n$ $=19$ ) groups stimulated continuously at $520 \mathrm{~nm}$ over a 5 -minute period (ON) in the BLA (two-way RM ANOVA time $x$ opsin interaction $F_{(2,70)}=3.32,{ }^{*} p=0.04$; post-hoc Student's t-test Jaws vs YFP: * $p(O N)$ $=0.038$; post-hoc Student's t-test with Bonferroni corrections Jaws * $p$ (5 vs 10 minutes $)=0.01,{ }^{*} p(10$ vs 15 minutes) $=0.02$ ) (B) Percentage of time spent in the EOM open arms for CatCh (blue, $n=18$ ) and YFP control (green, $n=19$ ) groups stimulated at $470 \mathrm{~nm}$ over a 5 -minute period (ON) at $10 \mathrm{~Hz}, 5 \mathrm{~ms}$ pulse in the BLA (Two-way ANOVA main effect of time $F_{(2,70)}=4.41,{ }^{*} p=0.016$, time $x$ opsin interaction $F_{(2,70)}=4.43,{ }^{*} p=0.015$; post-hoc Student's t-test YFP vs CatCh ${ }^{* *} p(O N)=0.009$, post-hoc Student's ttest with Bonferroni corrections CatCh ${ }^{* *} p(5$ vs 10 minutes $)=0.001 ;{ }^{*} p(10$ vs 15 minutes $\left.)=0.01\right)(\mathbf{C})$ Percentage of time spent in the EOM open arms for CatCh (blue, $n=13$ ) and control YFP (green, $n=14$ ) groups stimulated at $470 \mathrm{~nm}$ over a 5 -minute period (ON) at $10 \mathrm{~Hz}, 5 \mathrm{~ms}$-pulse in the NAcLSh, showing no difference between the CatCh and YFP groups. (D) Percentage of time spent in open arms in the EOM for CatCh (blue, $n=13$ ) and YFP (green, $n=9$ ) groups stimulated in the BLA throughout the test at 10 $\mathrm{Hz}$, 5-ms light-pulse, after all receiving a nicotine IP injection (CatCh vs YFP: two-way RM ANOVA main effect of time $F_{(2,40)}=4.92,{ }^{*} p=0.01$, time $x$ opsin interaction $F_{(2,40)}=3.74,{ }^{*} p=0.03$; one-way RM ANOVA YFP: $F_{(2,16)}=5.77,{ }^{*} p=0.01$; CatCh: $F_{(2,24)}=1.59, p=0.6$; post-hoc Student's t-test YFP vs CatCh at 9 minutes $\left.{ }^{* *} p=0.006\right)$. (E) Preference score in online place preference (OPP) paradigm for Jaws vs YFP groups bilaterally implanted in the BLA (top), for CatCh vs YFP groups bilaterally implanted in the BLA (middle), and for CatCh vs YFP groups bilaterally implanted in the NAcLSh (bottom). Animals were stimulated (continuously in the BLA, or at $10 \mathrm{~Hz}, 5$-ms light-pulse, in the NAcLSh) over the 20 minutetest, and the preference score is defined by the $\%$ of time spent in the compartment where the animals are photo-stimulated compared to the compartment where they are not (ON-OFF). (Top-down) Optical inhibition of the VTA-BLA pathway (orange, $n=17$ ) induced online place avoidance compared to the control group (YFP in green, $n=20$ ) (Student's t-test $\left.{ }^{*} p(O N-O F F)=0.02\right)$. Mice with optical activation of the VTA-BLA pathway (blue, $n=12$ ) did not display any difference in the OPP compared to the control group (YFP in green, $n=13$ ) (Student's t-test $p(O N-O F F)=0.5$ ). Optical activation of the VTA-NAcLSh pathway (blue, $n=13$ ) induced online place preference (Student's t-test * $p(O N-O F F)=0.04$ ) compared to the control group (YFP in green, $n=14$ ). 
(f)

Optical Virus fibers injection BLA $V$ VTA

D

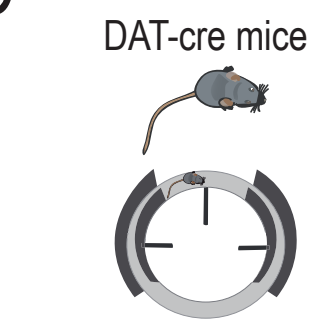

IP Nic $(0.5 \mathrm{mg} / \mathrm{kg})$

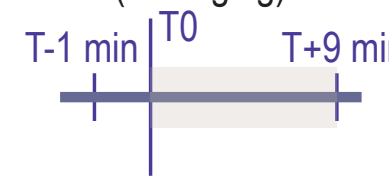

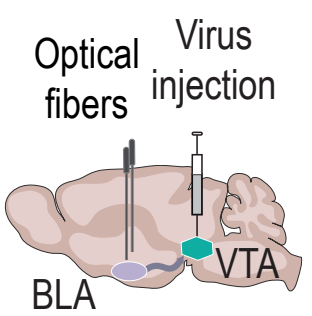

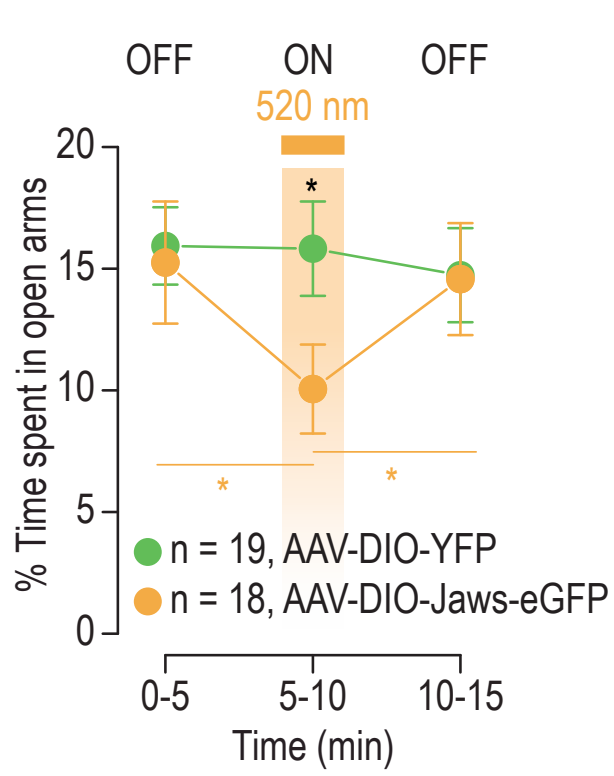

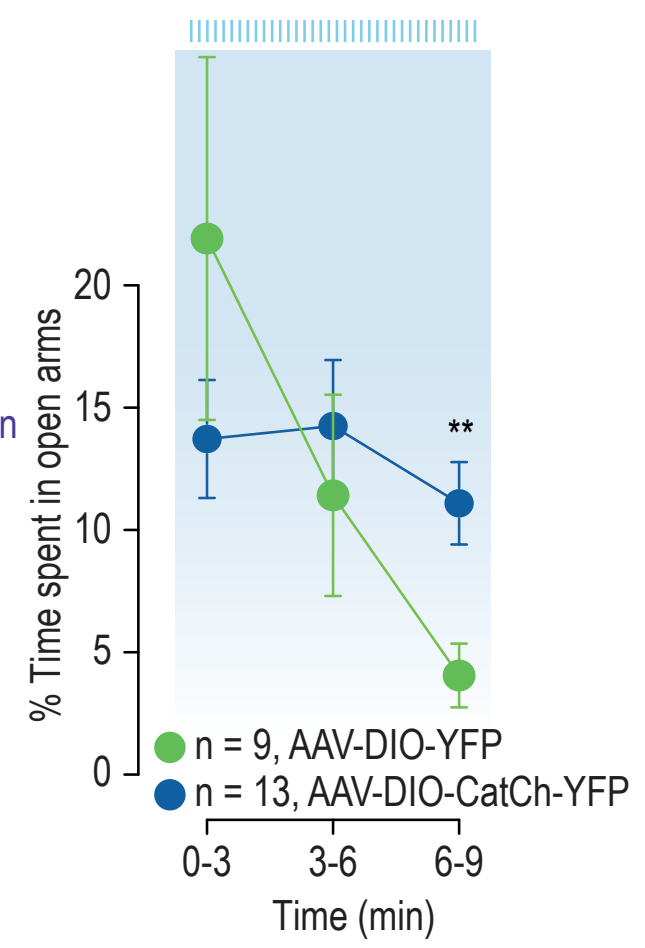

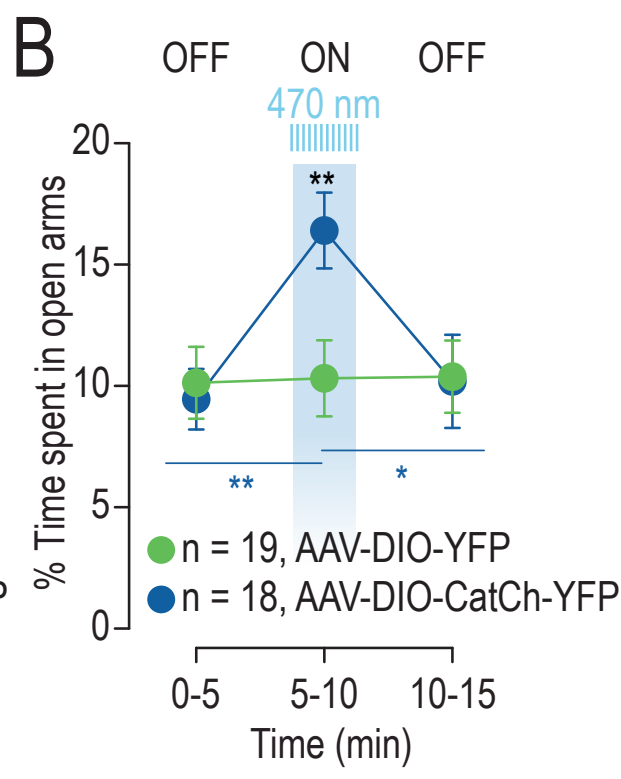
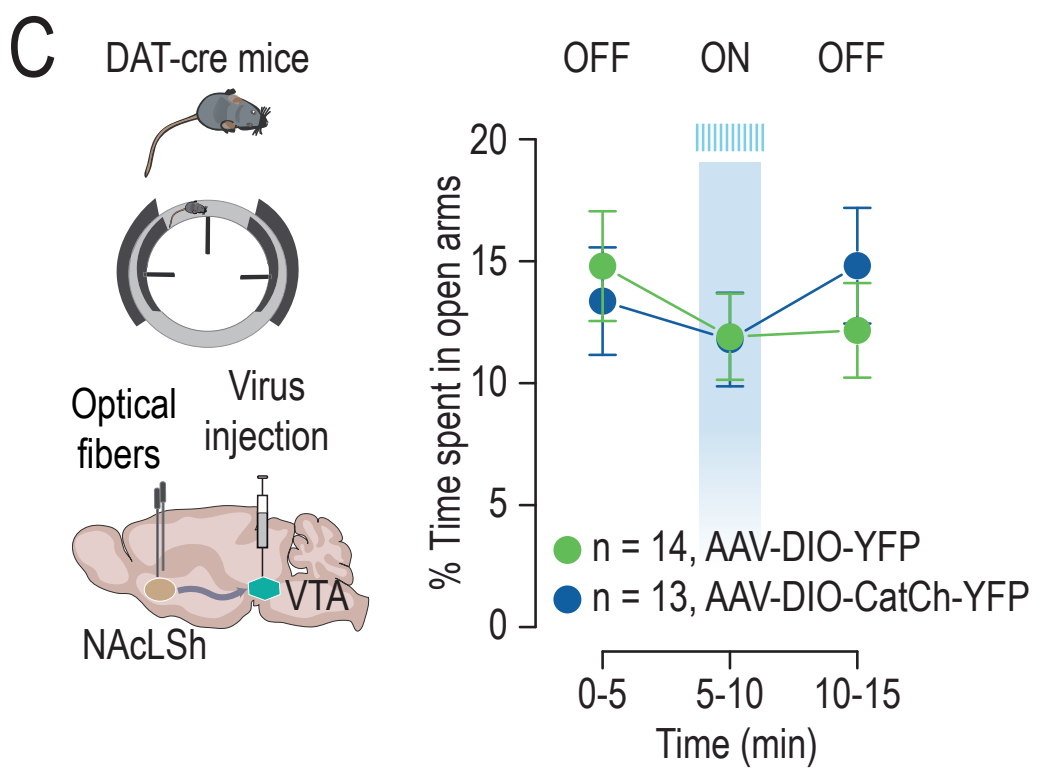

E
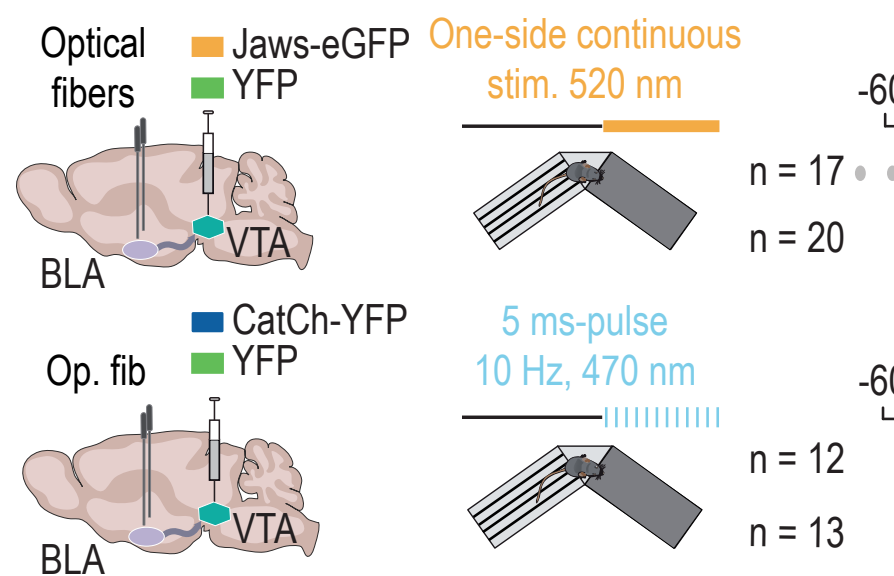

Preference score (ON-OFF \%)

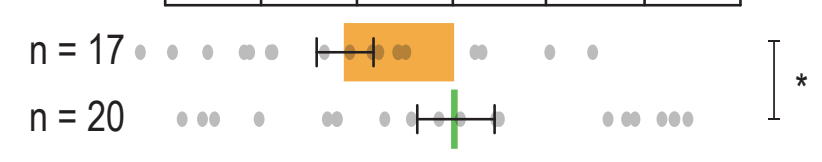

5 ms-pulse
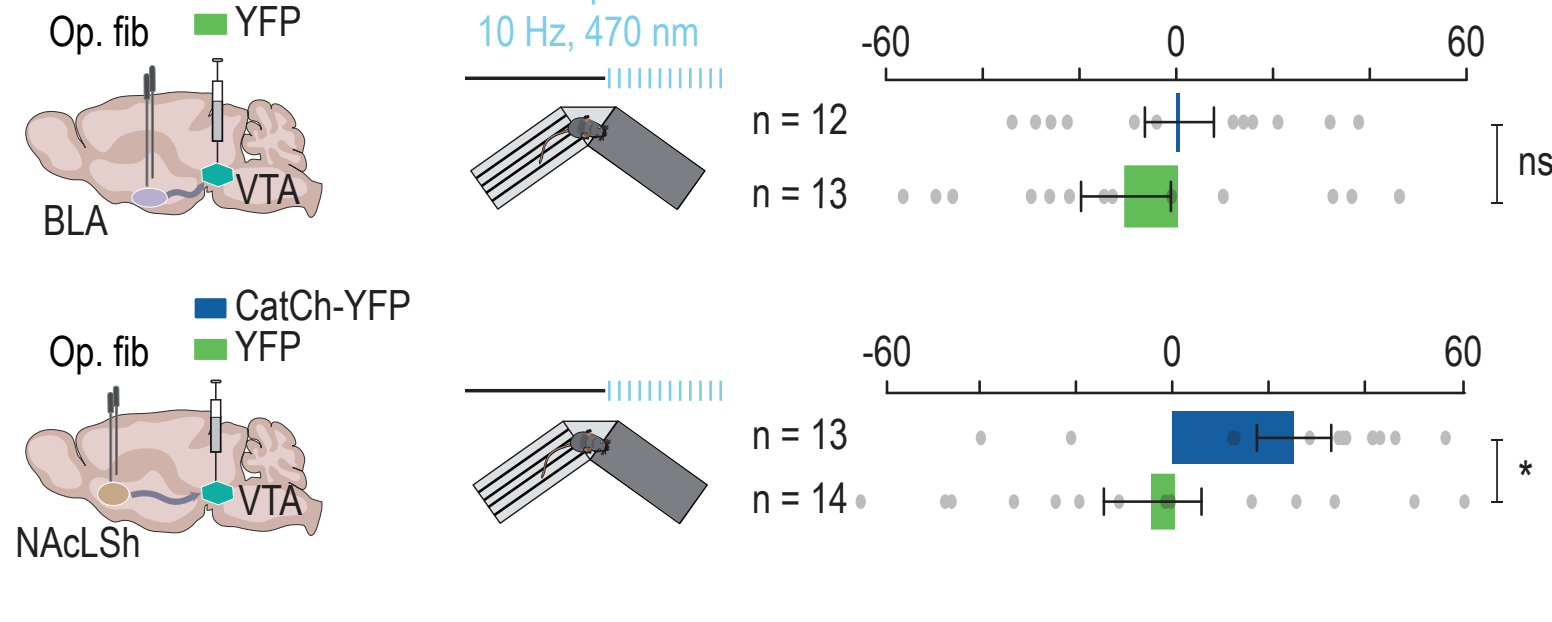

\section{Figure 4}




\section{Supplementary Figures}

\section{$430 \quad$ Figure $\mathbf{S 1}$}

431 (A-B) Principal component analysis (PCA) of the spontaneous activity of VTA DA neurons labeled in vivo 432 after juxtacellular recordings. First (axis Dim 1) and second (axis Dim 2) components are represented. 433 Based on their basal activity, neurons are distributed in 4 clusters (cluster $1, n=77$; cluster 2, $n=132$; 434 cluster $3, n=28$; cluster $4, n=7)(A)$, with nicotine-activated (in red, $n=154$ ) and nicotine-inhibited (in 435 blue, $n=90$ ) neurons found in each of these clusters $(B)$. (C) Mean firing frequency $(\mathrm{Hz})$ as a function of 436 percentage of spikes within a burst (\%SWB) for the 4 clusters found with the PCA analysis, with the 437 existence of nicotine-activated (cluster $1, n=52$; cluster $2, n=85$; cluster $3, n=15$; cluster $4, n=2$ ) and 438 nicotine-inhibited (cluster 1, $n=25$; cluster 2, $n=47$; cluster $3, n=13$; cluster $4, n=5$ ) DA neurons in all 439 of them. (D) Localization of VTA DA neurons labeled in vivo after juxtacellular recordings. Neurons are 440 color-coded according to their responses to nicotine injection (activated in red, $n=155$ and inhibited in 441 blue, $n=90$ ), and positioned according to the antero-posterior axis on the Paxinos atlas from Bregma $442 \quad 2.8$ to $-3.8 \mathrm{~mm}$. 
A

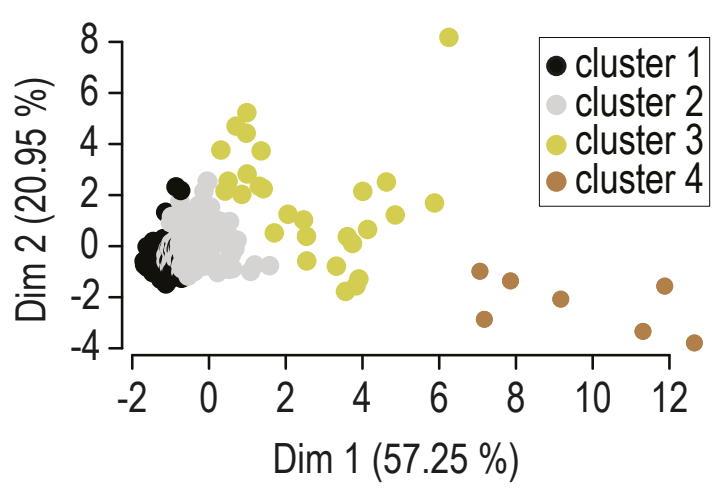

B

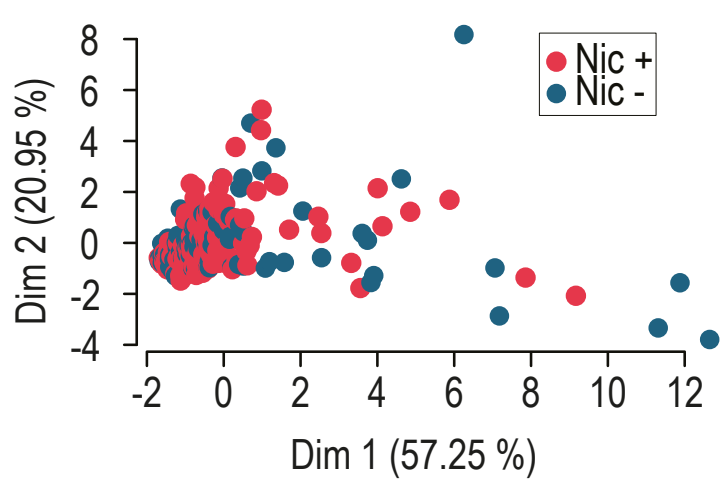

C

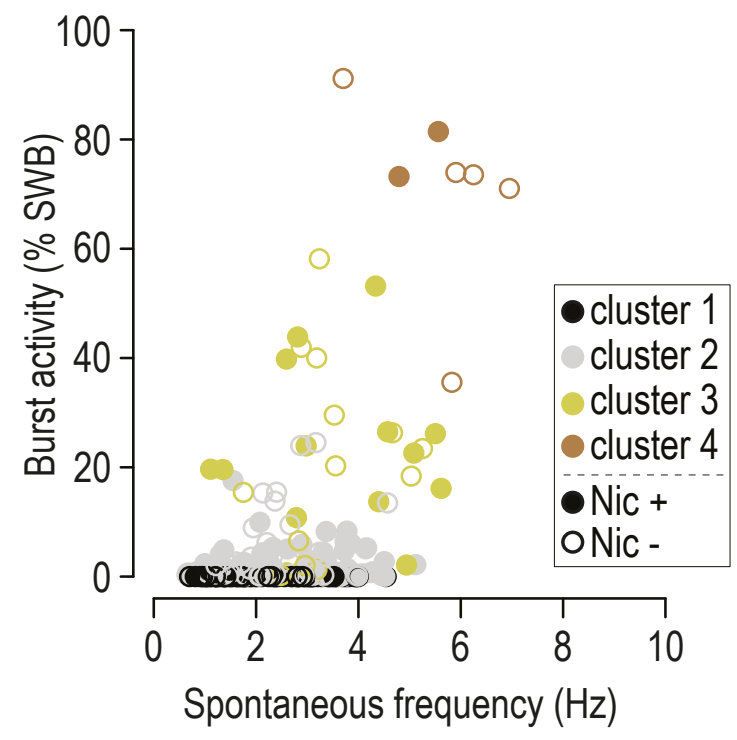

D

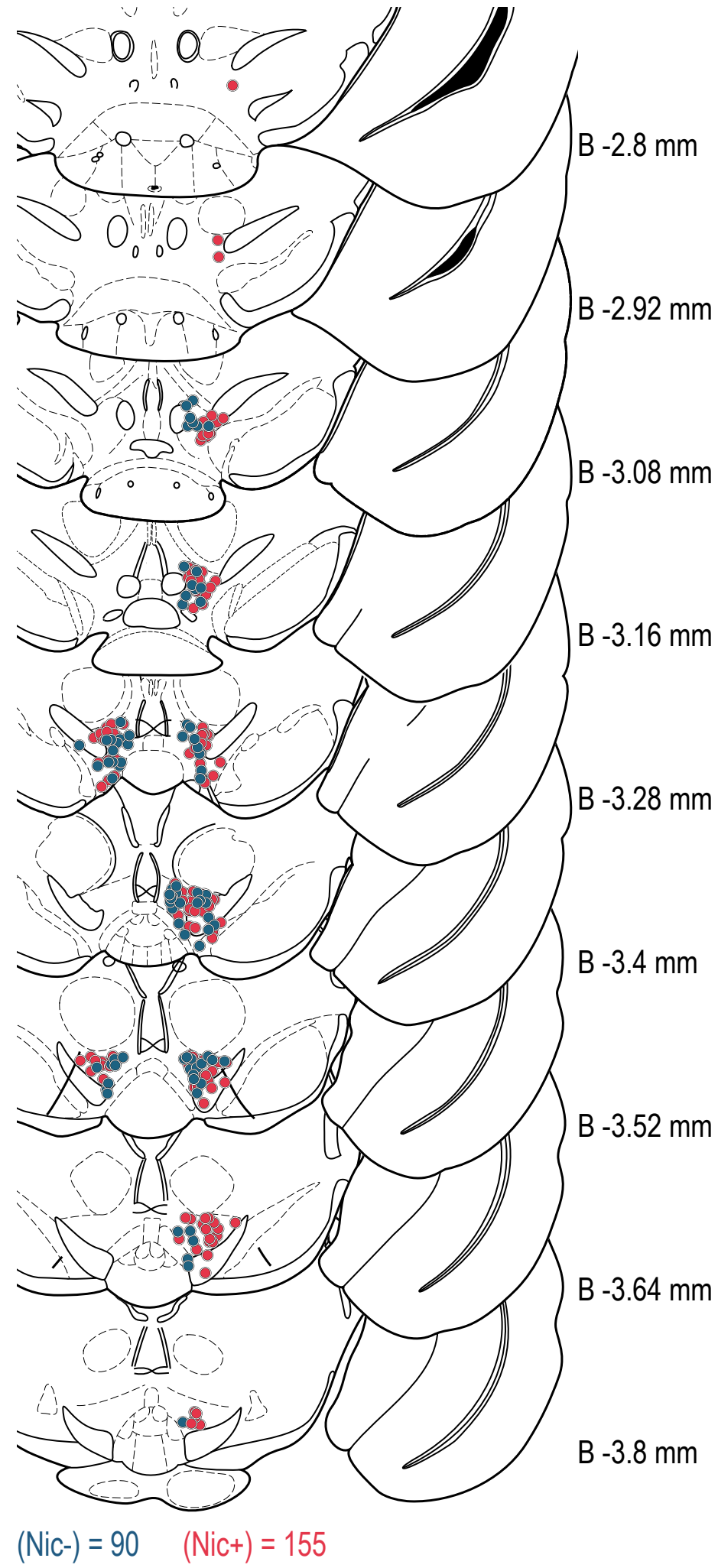




\section{$443 \quad$ Figure S2.1}

444 (A, B) Examples of NAc-injection sites (A) and Amg-injection sites (B) with retrobead (RB) tracer (in 445 yellow) reported onto Paxinos atlas slices. (C, D) Examples of immunohistofluorescence analysis of VTA 446 slices $(\mathrm{TH}+$, red) revealing neuronal soma containing $\mathrm{RB}(\mathrm{RB}+$, green), after injection of the retrobeads 447 into the NAc (C) or into the Amg (D). (E) A Paxinos atlas slice at $3.3 \mathrm{~mm}$ from bregma onto which 448 Neurobiotin-filled cell bodies of each recorded neuron were positioned. (F) Analysis of the medio-lateral 449 distribution of recorded DA neurons of the VTA (shown as density) projecting either to the NAc ( $n=30$, 450 gold) or to the Amg ( $n=22$, purple) revealed that Amg-projecting neurons are located more medially in 451 the VTA than NAc-projecting neurons (Wilcoxon test, ${ }^{* * *} p<0.001$ ).

452 IF: interfascicular nucleus; IPN: interpeduncular nucleus; ml: medial lemniscus; SNc: substantia nigra 453 pars compacta 
A
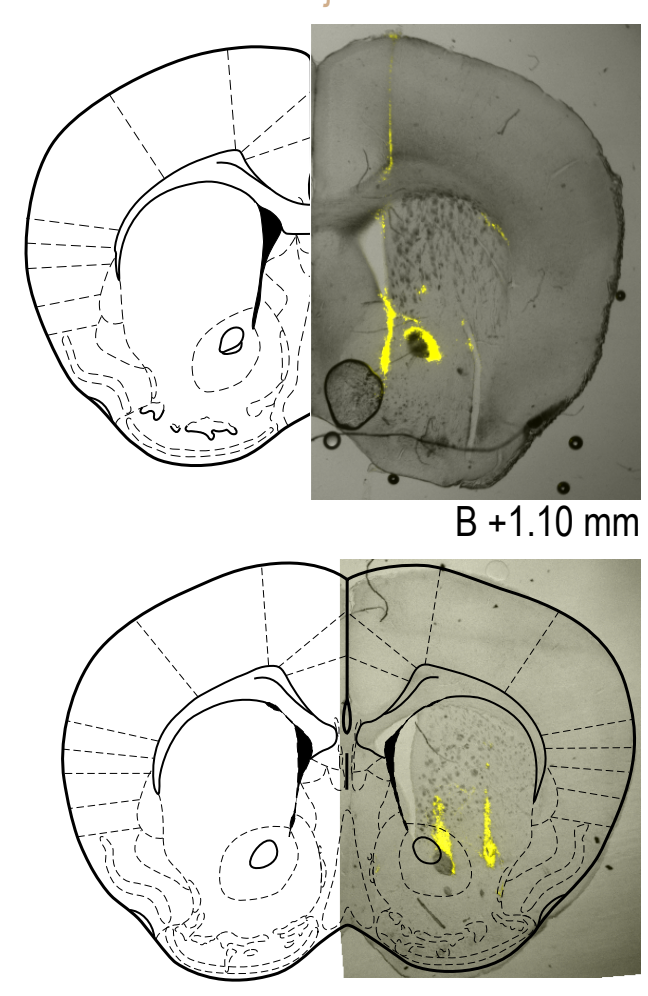

$\mathrm{B}+1.18 \mathrm{~mm}$

C $T H+R B+$

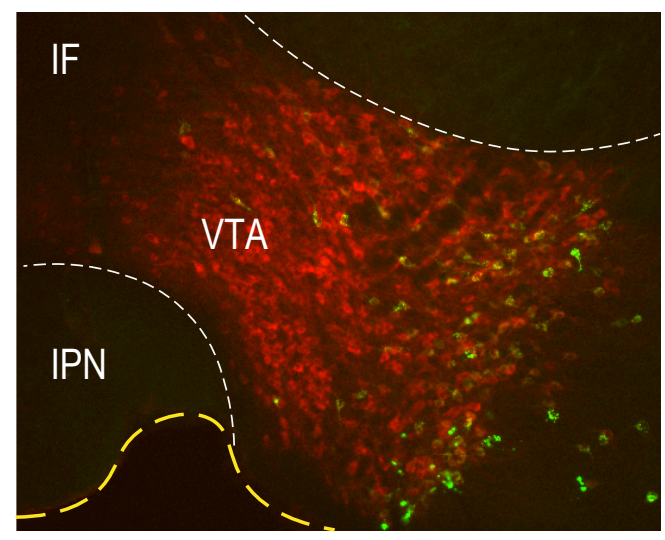

E

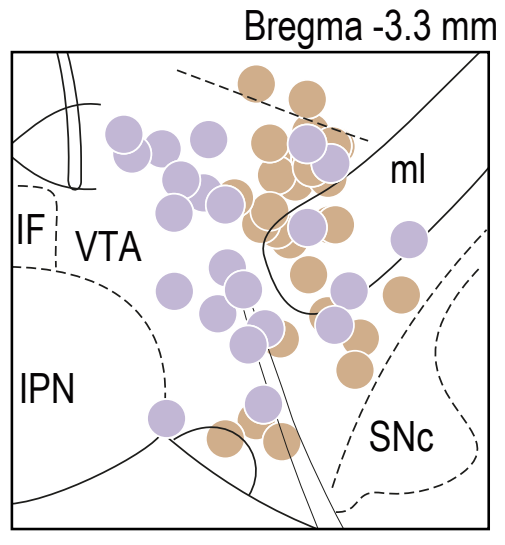

B Amg injections
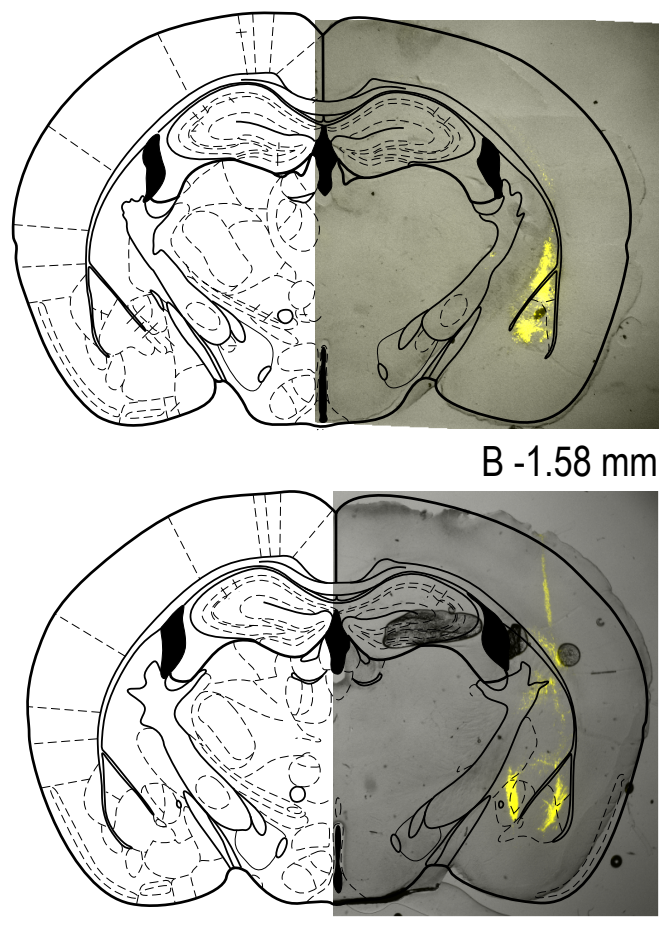

B - $1.46 \mathrm{~mm}$

D

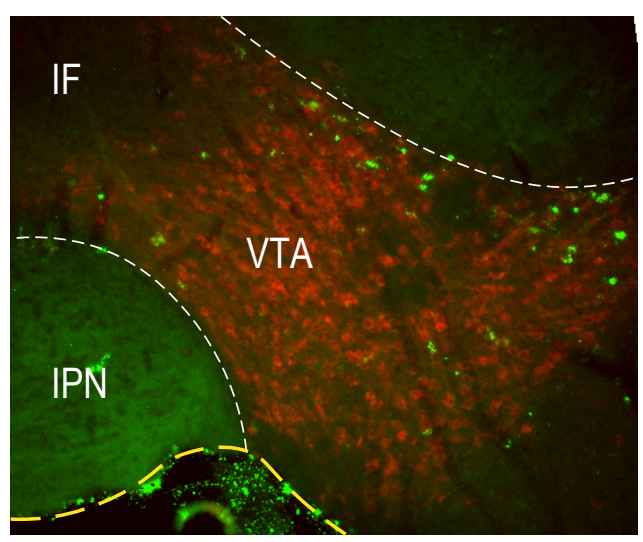

F

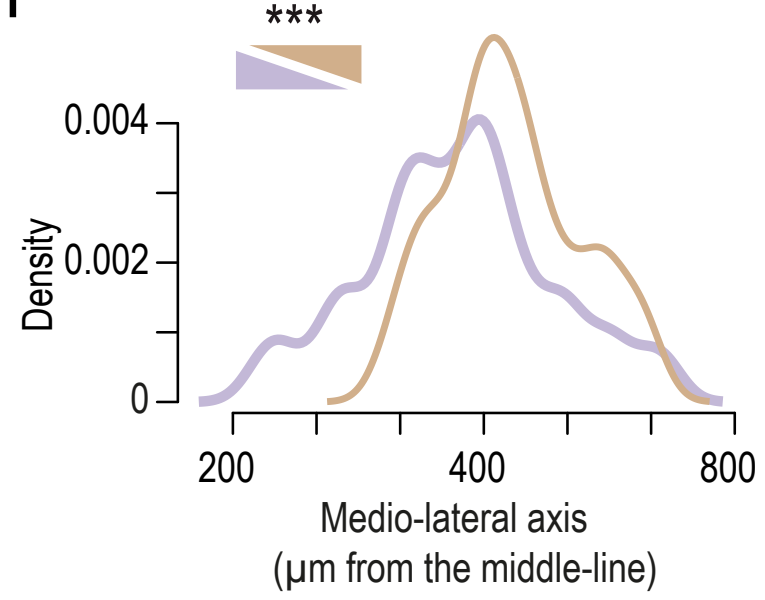




\section{Figure S2.2}

455 (A) Immunohistofluorescence analysis of a NAc-projecting neurons of the VTA labeled (neurobiotin, NB+) 456 after patch-clamp recording: confirmed as $\mathrm{DA}(\mathrm{TH}+)$ and NAc-projecting (retrobeads, $\mathrm{RB}+$ ). (B) 457 Immunohistofluorescence analysis of an Amg-projecting neuron of the VTA labeled (NB+) after patch458 clamp recording: confirmed as $\mathrm{DA}(\mathrm{TH}+)$ and Amg-projecting $(\mathrm{RB}+)$. (C) Firing of NAc-projecting and 459 Amg-projecting DA neurons of the VTA after injection of currents (20,60 and 100 pA). (D) Higher 460 excitability of Amg-projecting ( $n=17$, purple) compared to NAc-projecting DA neurons ( $n=16$, gold) (two461 way RM ANOVA main effect phenotype $F_{(1,26)}=4.96$, ${ }^{*} p=0.035$, current $F_{(4,104)}=15.97$, ${ }^{* * *} p<0.001$, 462 current $x$ phenotype interaction $F_{(4,104)}=13.78$, ${ }^{* * *} p<0.001$ ). (E) Nicotine-evoked currents (local puff 100 $463 \mu \mathrm{M}$ ) in RB+-identified, NAc- or Amg-projecting VTA DA neurons recorded in brain slices (whole-cell 464 voltage-clamp mode -60 mV). (F) Mean currents evoked by nicotine in either NAc-projecting ( $n=16$, gold, $46533.0 \pm 19.8 \mathrm{pA}$ ) or Amg-projecting ( $\mathrm{n}=17$, purple, $22.4 \pm 13.3 \mathrm{pA}$ ) VTA DA neurons are not statistically 466 different (Student's t-test $p=0.08)$. 
A

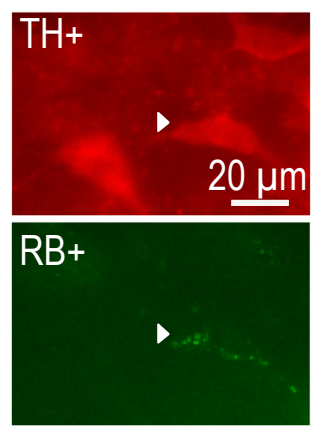

C

$$
+20 p A+60 p A+100 p A
$$
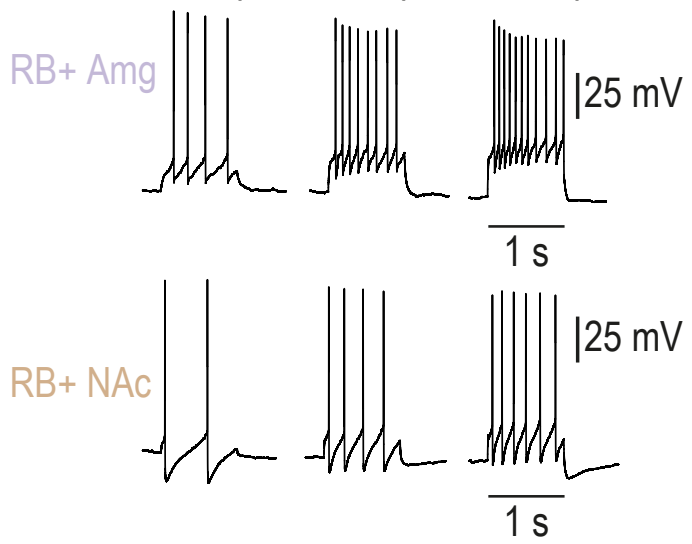

$E$

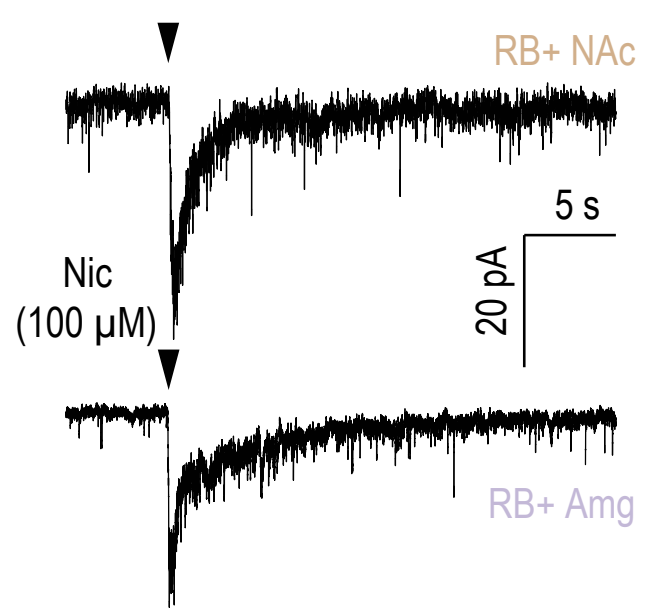

B

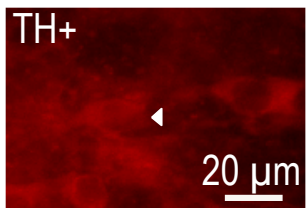

$\mathrm{NB}+$
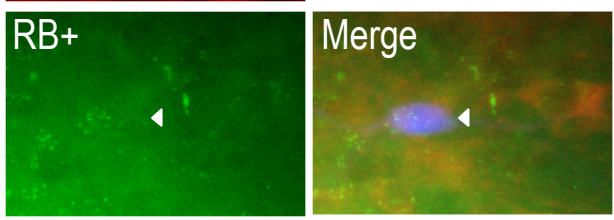

$D$

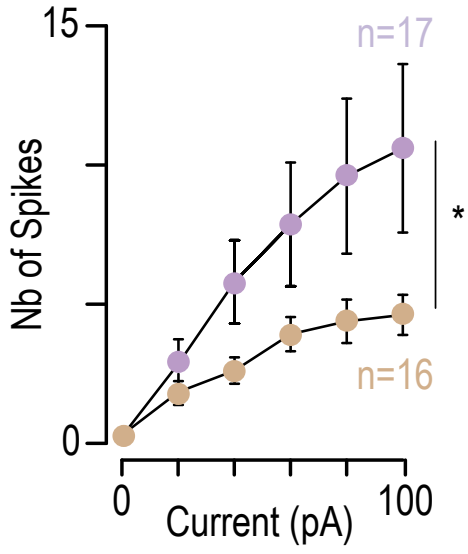

F

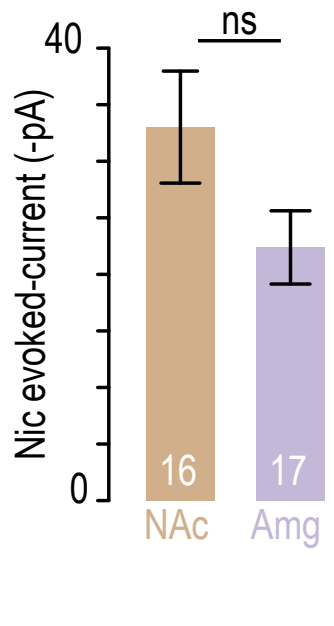




\section{Figure S3}

468 (A) Locomotor activity, measured in a square open field (OF), of mice receiving an intraperitoneal (IP) 469 injection of either saline (IP Sal, grey, $n=11$ ) or nicotine (IP Nic $0.5 \mathrm{mg} / \mathrm{kg}$, black, $n=7$ ). Mice did not 470 display any statistically significant difference in the distance traveled over time (pooled each 3 minutes, 471 two way RM ANOVA no time, no treatment or interaction effect $p>0.05$ ) or in the total distance traveled 472 during 9 minutes (presented as bar plot, Student's t-test $p>0.05$ ) (B) EOM detailed for WT mice tested 473 with Sal (grey, $n=23$ ) or Nic (black, $n=21$ ) (one-way RM ANOVA Nic: $F_{(2,40)}=5.18,{ }^{* *} p=0.01$; Sal: $\left.474 F_{(2,44)}=1.65, p=0.2\right)$ after IP injection. Mean scores are represented in bold and color, and individual 475 scores with empty grey dots. (C) Example of post-hoc verification of intracranial guide cannula 476 implantations in WT mice. Bilateral injection cannulas $(0.5 \mu \mathrm{m}$ longer than the guide cannulas) are 477 inserted on the day of the experiment, for local infusion into the VTA. TH labeling is shown in yellow. (D) 478 Detailed scores of wild-type mice in the EOM after intracranial infusion of Sal (grey, $n=6$ ) or Nic (red, $n$ $479=7) 1 \mathrm{mg} / \mathrm{mL}$ (one-way RM ANOVA Nic: $F_{(2,12)}=26.11$, ${ }^{* * *} p<.001 ;$ Sal: $\left.F_{(2,10)}=0.01, p=0.99\right) 1$ minute 480 before the test. Mean scores are represented in bold and color, and individual scores in empty grey dots. 481 (E) Schematic of $\beta 2$ subunit re-expression by lentiviral vectorization in the VTA of $\beta 2^{-/-}$mice. Lentivirus 482 encoding either $p G K-\beta 2-I R E S-G F P\left(\beta 2^{-/-V e c}\right)$ or $p G K-G F P\left(\beta 2^{-I-G F P}\right)$ as a control were injected into the 483 VTA. Example of immunohistofluorescence analysis of a $\beta 2^{-/-V e c}$ mouse brain labeled for TH (red) and 484 GFP (green). (F) The percent of time spent in the open arms of the EOM in $\beta 2^{-1-G F P}$ animals $(n=6$, 485 green) and $\beta 2^{--}$mice $(n=17$, grey) are not different (two-way RM ANOVA no time, no treatment or 486 interaction effect $p>0.05)$. (G) Detailed scores in the EOM test of $\beta 2^{-/-}$mice $\left(n=23\right.$, green) and $\beta 2^{-/-V e c}$ 487 mice $\left(n=18\right.$, brown), after an injection of Nic $0.5 \mathrm{mg} / \mathrm{kg}$ (one-way RM ANOVA $\beta 2--\mathrm{Vec}$ : $\mathrm{F}_{(2,34)}=8.65$, *** $\left.488 p<0.001 ; \beta 2^{--:} F_{(2,44)}=1.08, p=0.3\right) 1$ minute before the test. Mean scores are represented in bold and 489 color, and individual scores in empty grey dots.

490 IF: interfascicular nucleus ; IPN : interpeduncular nucleus ; SNc : substantia nigra pars compacta ; ml : 491 medial lemniscus ; SNc : substantia nigra pars compacta. 
bioRxiv preprint doi: https://doi.org/10.1101/2020.07.30.228189; this version posted July 30, 2020. The copyright holder for this preprint

A

(which was not certified by peer review) is the author/funder. All rights resgrved. No reuse allowed without permission.

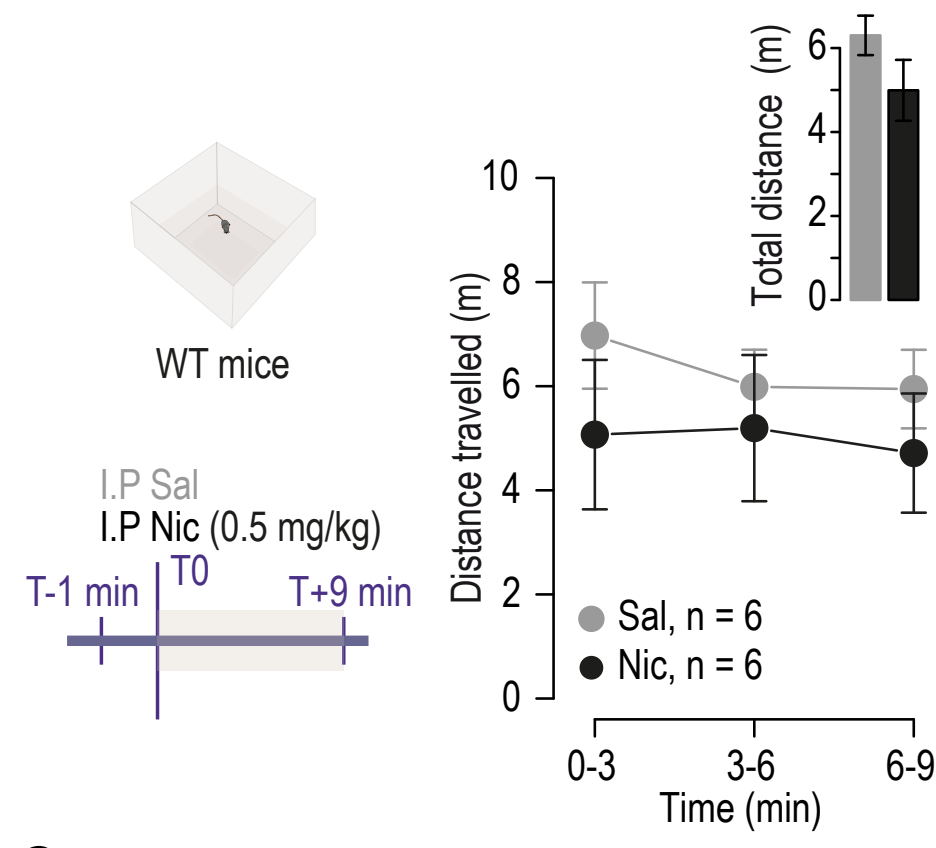

C

Implant location of bilateral guide cannulas
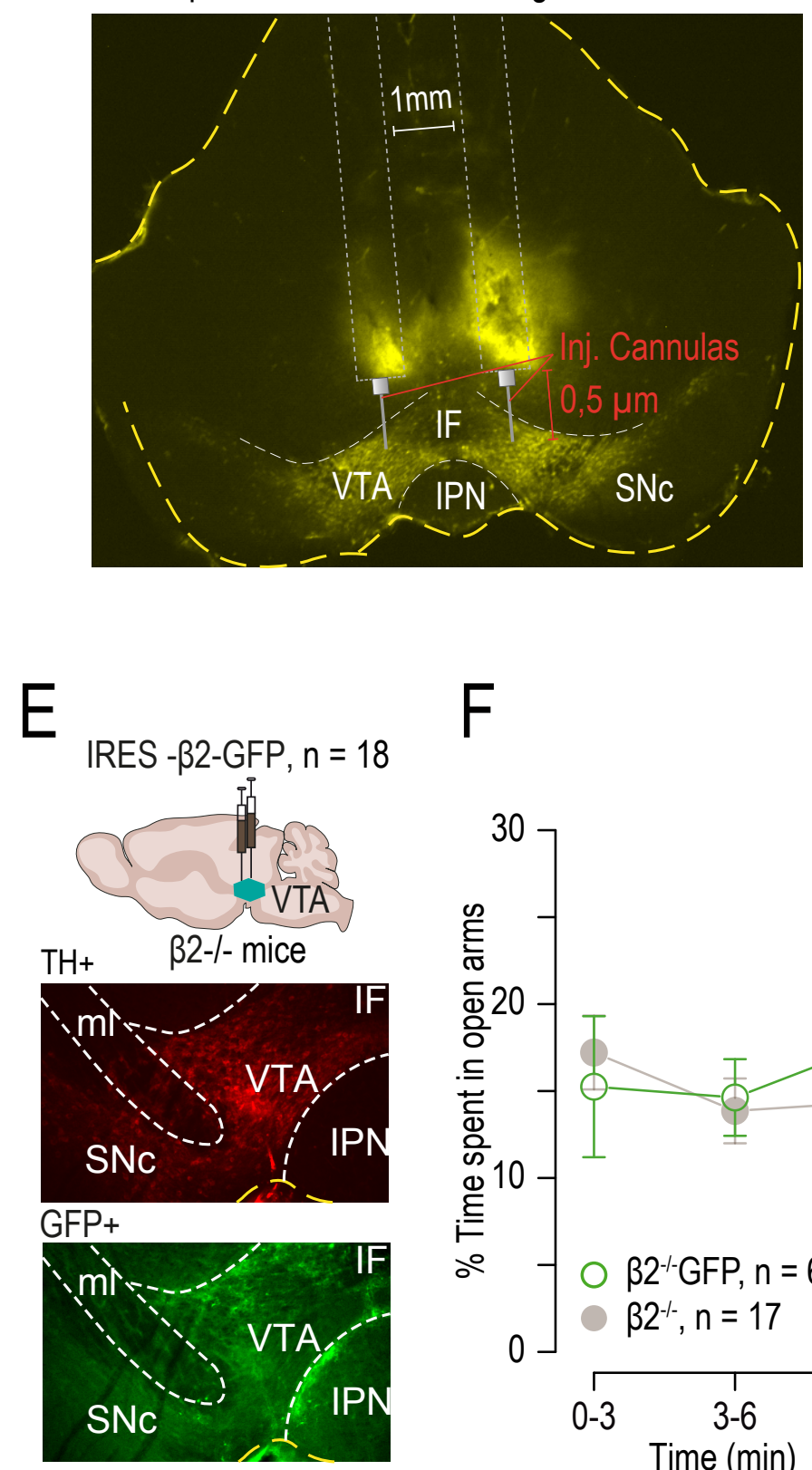

$F$

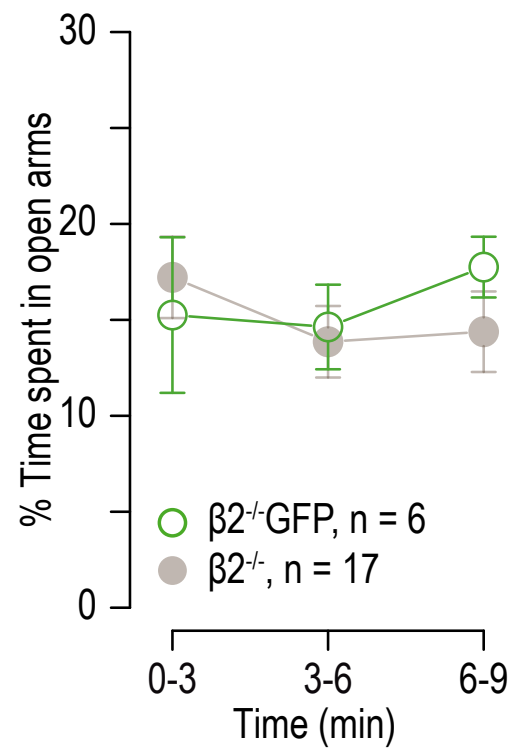

0

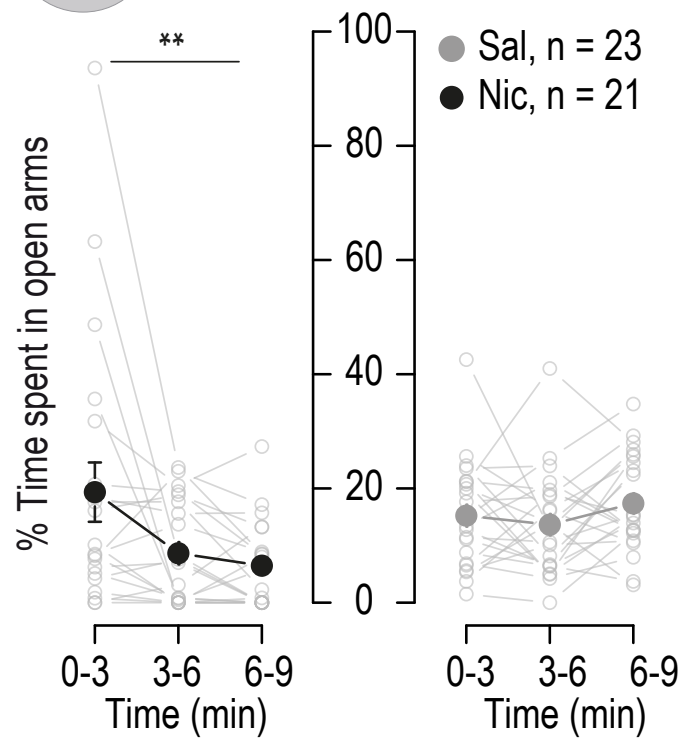

Nic, IP $0.5 \mathrm{mg} / \mathrm{kg}$

Time (min)

$D$

(- Nic, IC $100 \mathrm{ng}$ (100nl infusion)

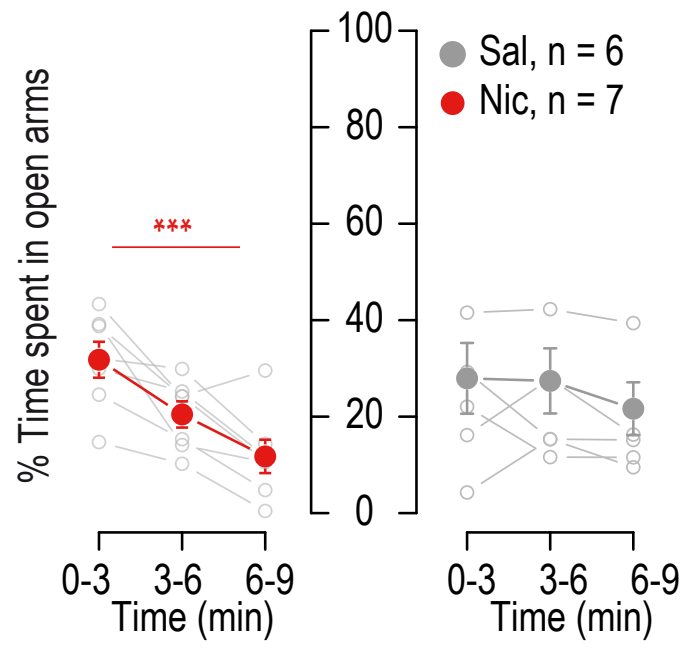

$G$
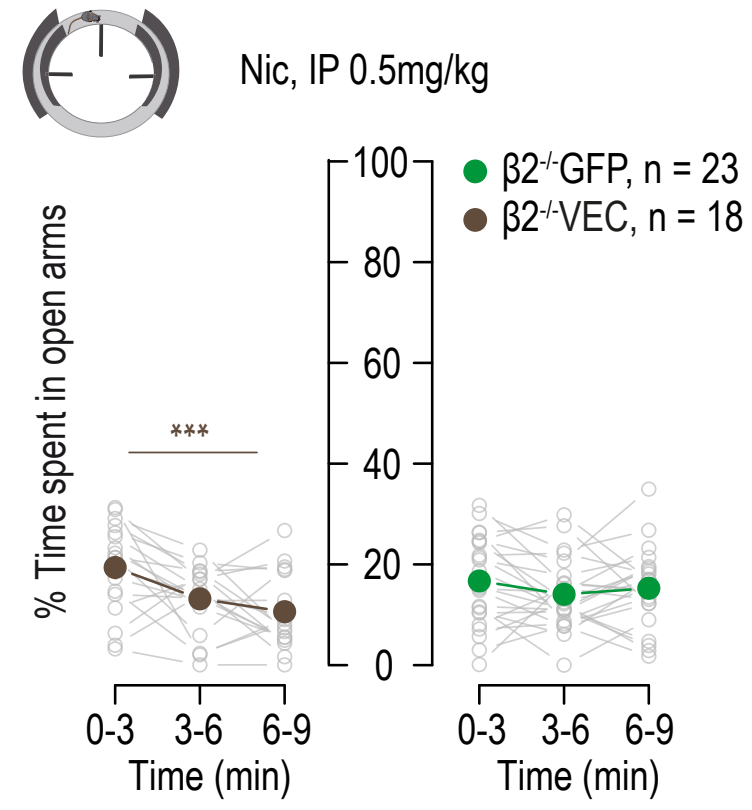


\section{Figure S4.1}

493 (A) Immunohistofluorescence analysis of VTA DA neurons after patch-clamp recordings in mice injected 494 with AAV-Ef1 $\alpha$-DIO-Jaws-eGFP into the VTA. Neurobiotin (NB, blue), tyrosine hydroxylase (TH, red), 495 YFP (green). (B) Example of a recording trace of a VTA DA neuron during continuous light stimulation $496(20 \mathrm{~s}, 520 \mathrm{~nm})$ and raster plot of action potential showing light-induced inhibition in Jaws-expressing DA 497 neurons $(n=7)$. (C) Immunohistochemistry of VTA DA neurons (NB, blue; TH, red; YFP, green) after 498 patch-clamp recording in mice injected with AAV-DIO-CatCh-YFP into VTA. (D) Example of recording 499 trace of a DA neuron of the VTA during light stimulation (10 Hz, 5-ms pulse, $470 \mathrm{~nm})$ and light-evoked 500 inward current in DA neurons expressing CatCh. Mean light-evoked current in seven DA neurons. 
A
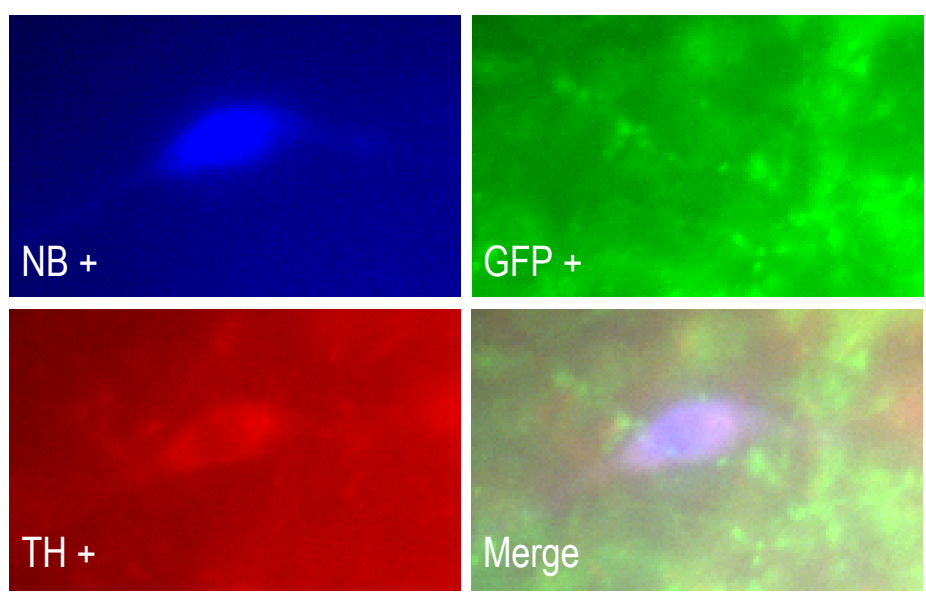

C
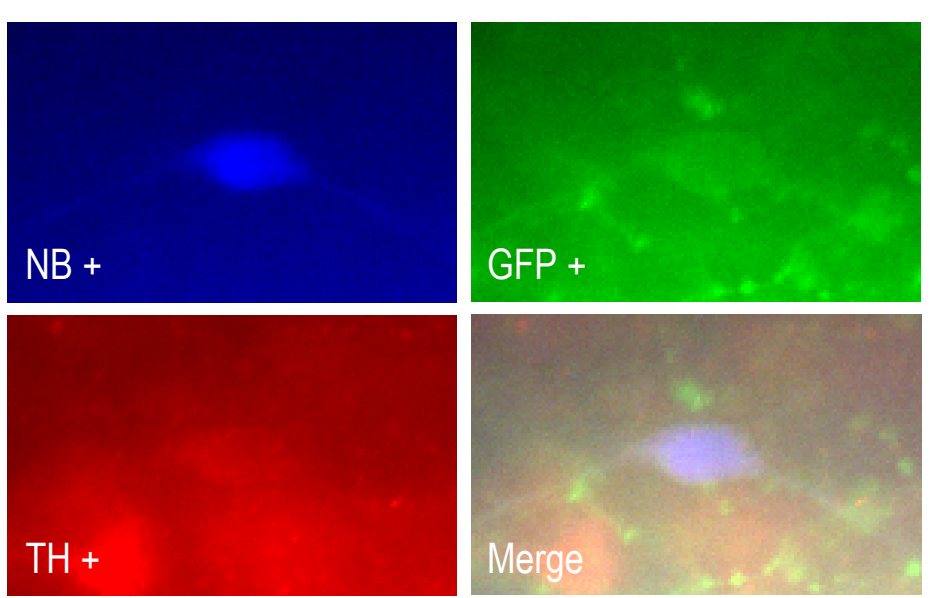

B
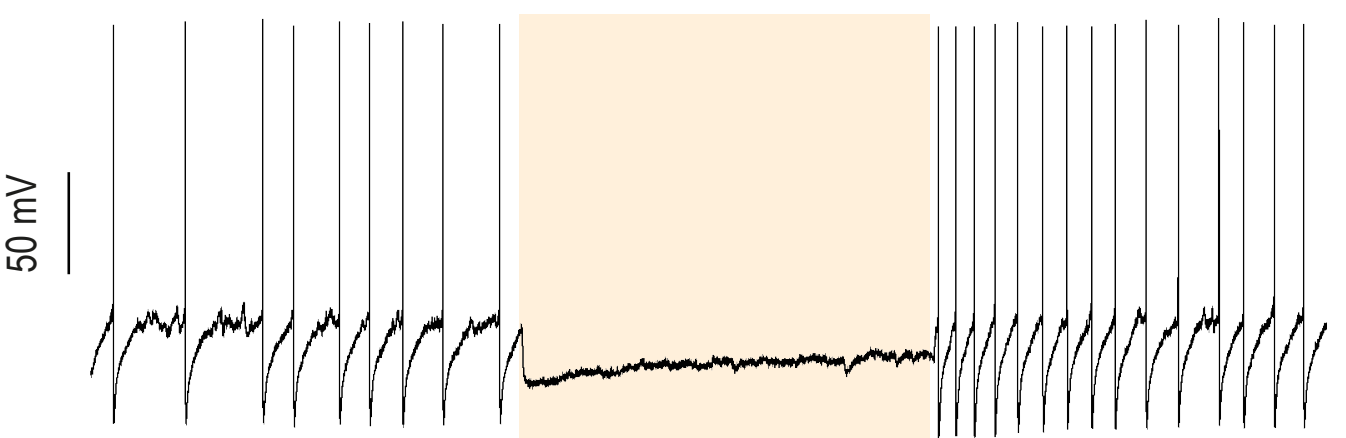

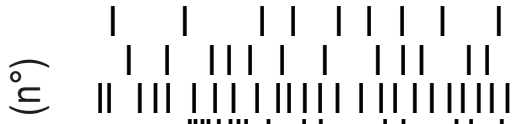

¿

옹 | | | || ||||| |||| | | | | | | |

\& $\frac{1}{\Gamma_{0}}$

20

IIIIIII|| | |1 |

I III | I| | III II I I

III I I

|||| |||| | || | | ||| | || ||

IIII || | | || || |

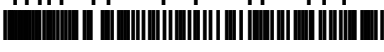

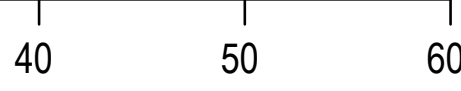

D

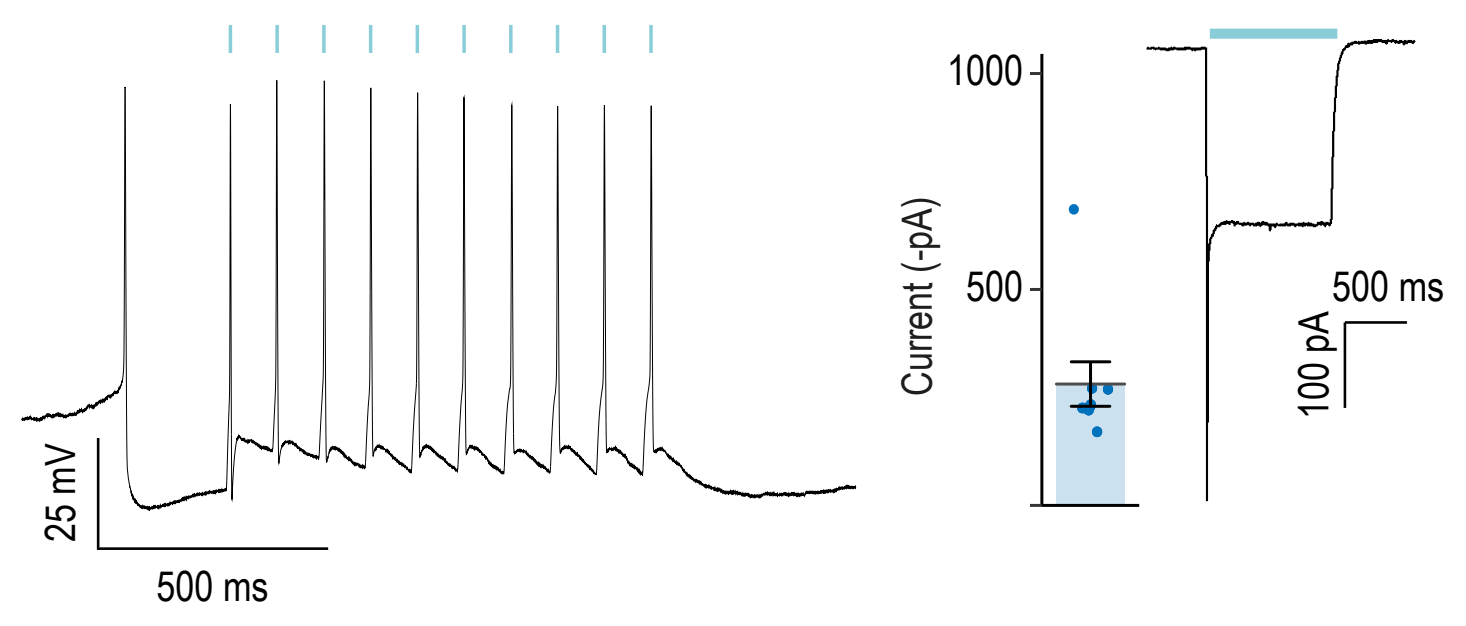

Figure S4.1 


\section{$501 \quad$ Figure $\mathbf{S 4 . 2}$}

502 (A) Immunohistofluorescence analysis of VTA slices after AAV-DIO-Jaws-eGFP and AAV-DIO-YFP 503 injections into VTA. (B) Immunohistofluorescence analysis of VTA slices after AAV-Ef1 $\alpha$-DIO-hCatCh504 YFP and AAV-Ef1 $\alpha$-DIO-YFP injections into the VTA. (C) Example of post-hoc verification of the fiber 505 implantation into the basolateral Amg (BLA) of mice used in optogenetic experiments, injected either with 506 AAV-Ef1 $\alpha$-DIO-Jaws-eGFP (orange dots indicate the location of one hemisphere fiber tip that was 507 verified, $n=13$, left side of the slices) and its YFP control (green dots, $n=10$, left side of the slices) or 508 with AAV-Ef1 $\alpha$-DIO-hCatCh-YFP (blue dots, $n=16$, right side of the slices) and its YFP control (green 509 dots, $n=11$, right side of the slices). The optical fibers were positioned onto Paxinos atlas slices from 510 bregma -1.22 to $-1.82 \mathrm{~mm}$. (D) Post-hoc verification of the fiber implantation into the NAc lateral shell 511 (NAcLSh) of mice used in optogenetic experiments, injected either with AAV-Ef1 $\alpha$-DIO-hCatCh-YFP 512 (blue dots, $n=13$, left of the slices) and its YFP control (green dots, $n=14$, right of the slices). The optical 513 fibers were positioned onto Paxinos atlas slices from bregma +0.86 to $+1.70 \mathrm{~mm}$. 
A

AAV-DIO-Jaws-eGFP
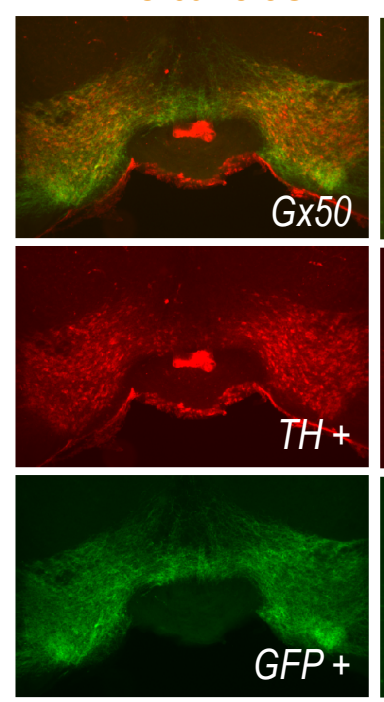

C

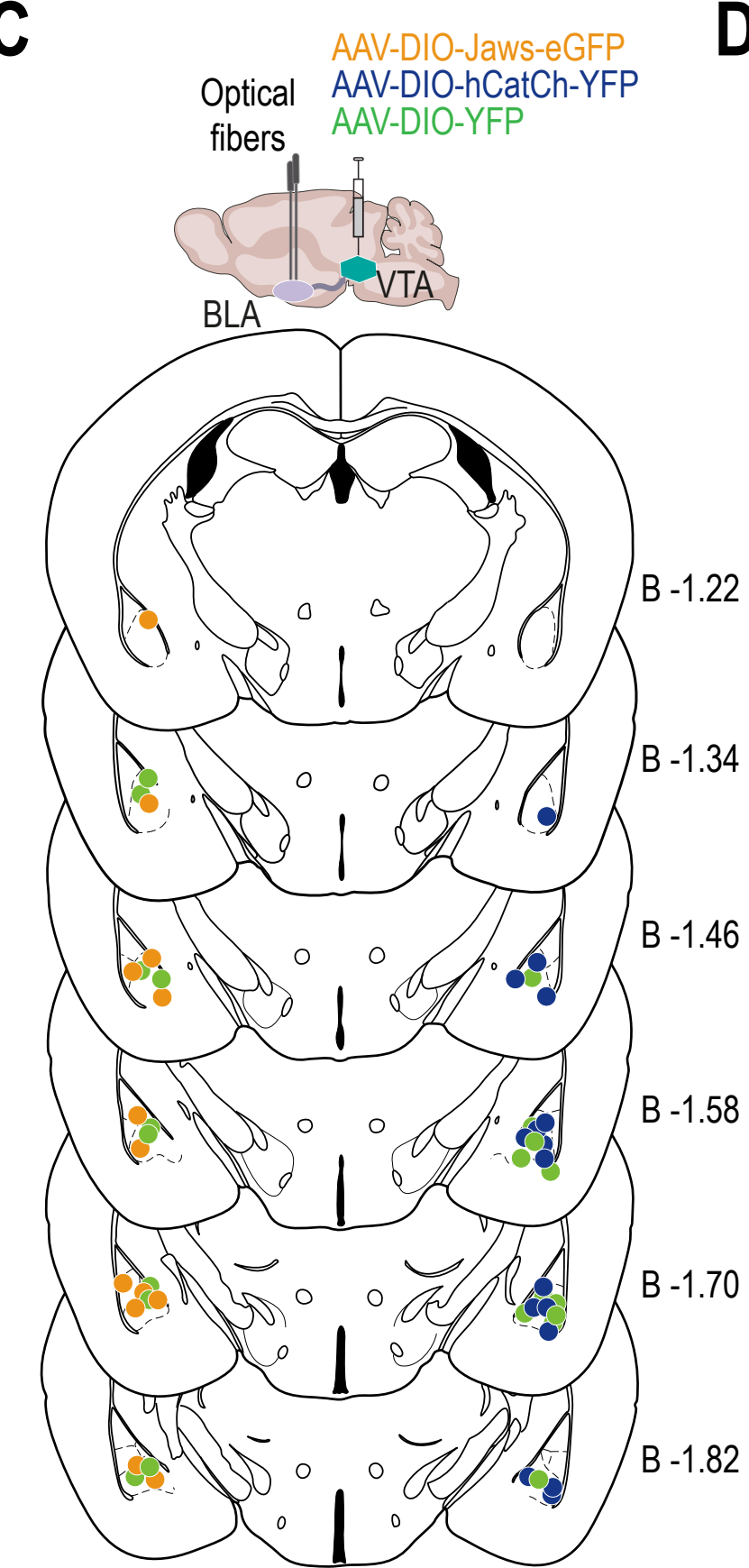

AAV-DIO-hCatCh-YFP
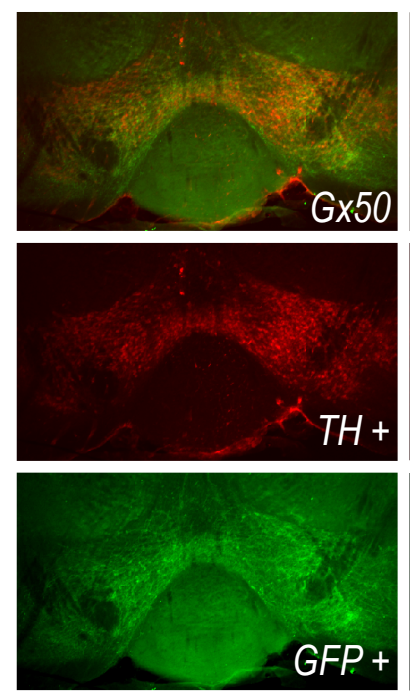

D

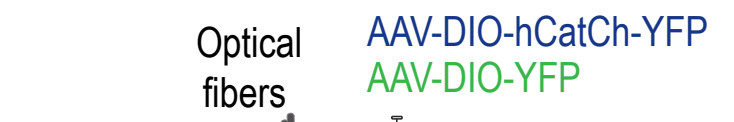

$\begin{array}{ll}\text { Optical } & \text { AAV-DIO-hCatCh-YFP } \\ \text { fibers } & \text { AAV-DIO-YFP }\end{array}$

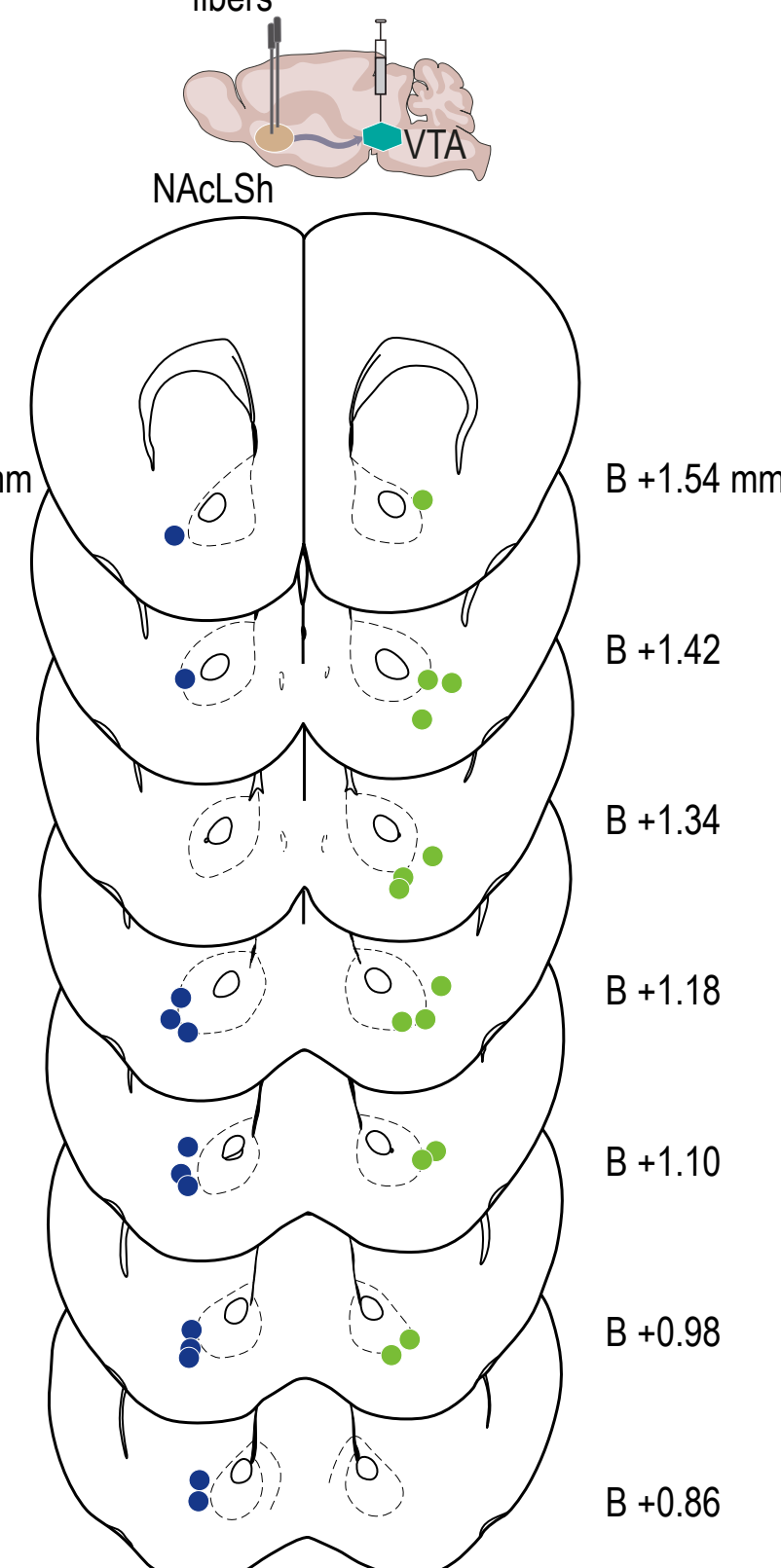

Figure S4.2 


\section{Figure S4.3}

515 (A) Time spent in open arms of the EOM by mice injected with AAV-Ef1 $\alpha$-DIO-Jaws-eGFP ( $n=18$, 516 orange, one-way RM ANOVA $\left.F_{(2,34)}=5.28,{ }^{* *} p=0.01\right)$ and AAV-Ef1 $\alpha$-DIO-YFP $(n=19$, green, one-way 517 RM ANOVA $\left.F_{(2,36)}=0.32, p=0.7\right)$ in the VTA, with (ON) or without (OFF) light-evoked inhibition of DA 518 axon terminals in the BLA (the test lasts 15 minutes, with continuous photostimulation at $520 \mathrm{~nm}$ during 519 a 5-minute ON period). Mean scores are represented in bold and color, and individual scores in grey. (B) 520 Time spent in open arms of the EOM by mice injected with AAV-Ef1 $\alpha$-DIO-hCatCh-YFP $(n=18$, blue, 521 one-way RM ANOVA $\left.F_{(2,34)}=9.27,{ }^{* * *} p<0.001\right)$ and AAV-Ef1 $\alpha-D I O-Y F P(n=19$, green, one-way 522 ANOVA $\left.F_{(2,36)}=0.01, p=0.99\right)$ in the VTA, with (ON) or without (OFF) light-evoked activation of DA axon 523 terminals in the BLA (10 Hz photostimulation at $470 \mathrm{~nm}, 5$-ms pulse, during a 5-minute $\mathrm{ON}$ period). Mean 524 scores and individual scores are shown as in (A). (C) Time spent in open arms of the EOM by mice 525 injected with AAV-Ef1 $\alpha$-DIO-hCatCh-YFP ( $n=13$, blue, one-way RM ANOVA $\left.F_{(2,24)}=0.61, p=0.55\right)$ and 526 AAV-Ef1 $\alpha$-DIO-YFP $\left(n=14\right.$, green, one-way RM ANOVA $\left.F_{(2,26)}=1.47, p=0.25\right)$ in the VTA, during (ON) 527 or out (OFF) light-evoked activation of DA axon terminals in the NAcLSh (10 Hz photostimulation at 470 $528 \mathrm{~nm}, 5$-ms pulses, during a 5-minute ON period). Mean scores and individual scores are presented as 529 previously described. (D) A square open field (OF) paradigm was conducted in the three paired groups 530 of animals to assess their locomotor activities: Jaws- and YFP-injected mice implanted in the Amg (two531 way RM ANOVA, main effect epoch $\mathrm{F}_{(2,76)}=44.27$, ${ }^{* * *} p<0.001$, no opsin or interaction effect). (E) CatCh532 and YFP-injected mice implanted in the BLA (two-way RM ANOVA, main effect epoch $\mathrm{F}_{(2,42)}=25.17$, *** $533 p<0.001$, no opsin or interaction effect). (F) CatCh- and GFP-injected mice implanted in the NAcLSh 534 (two-way RM ANOVA, main effect epoch $F_{(2,50)}=14.27$, ${ }^{* * *} p<0.001$, epoch $x$ opsin interaction $F_{(2,50)}=4$, $535{ }^{*} p=0.02$, post-hoc Wilcoxon test YFP vs CatCh at 5 minutes ${ }^{*} p=0.04$ ). Animals showed no difference 536 in terms of locomotor activity between the two groups or as a function of the stimulation period within a 537 group. 
bioRxiv preprint doi: https://doi.org/10.1101/2020.07.30.228189; this version posted July 30, 2020. The copyright holder for this preprint (which was not certified by peer review) is the author/funder. All rights reserved. No reuse allowed without permission.

A

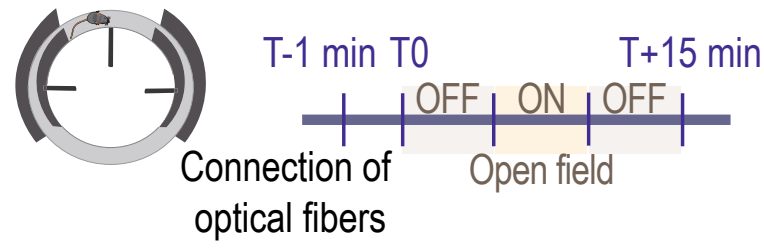

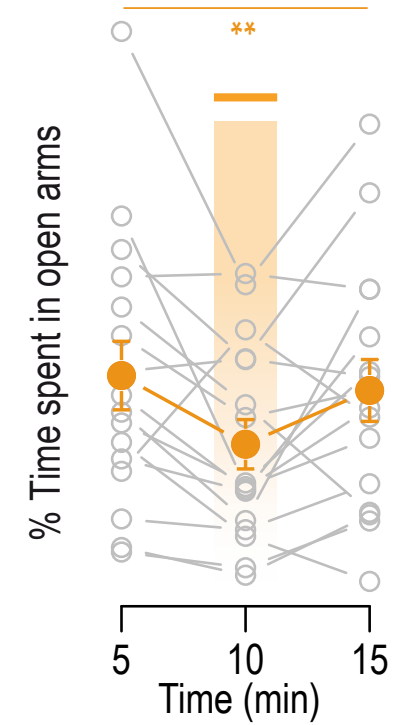

B

C

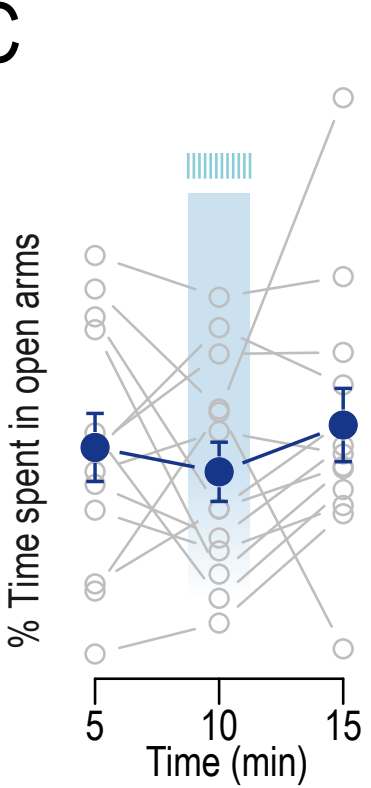

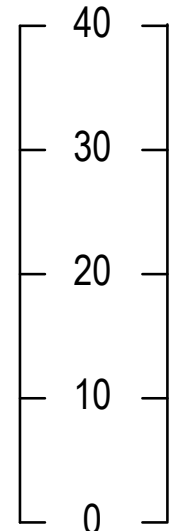
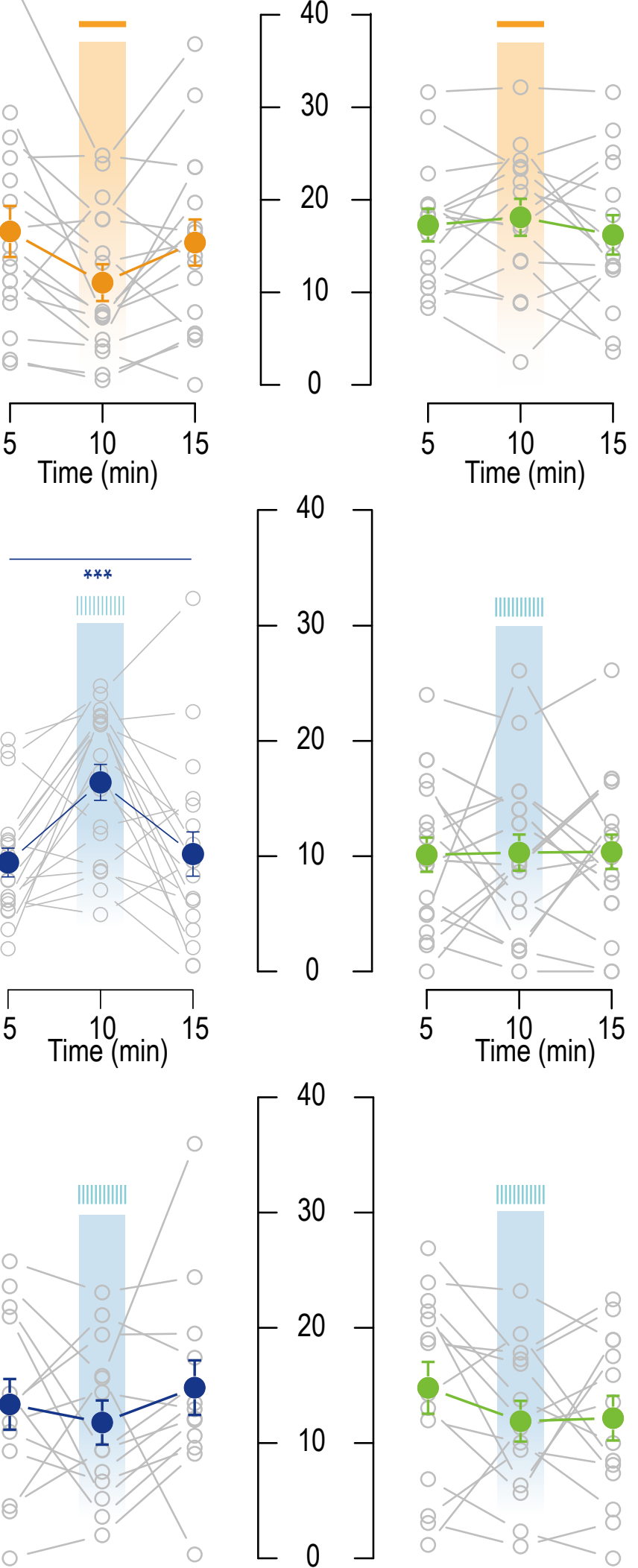

$\mathrm{F}$

$E$
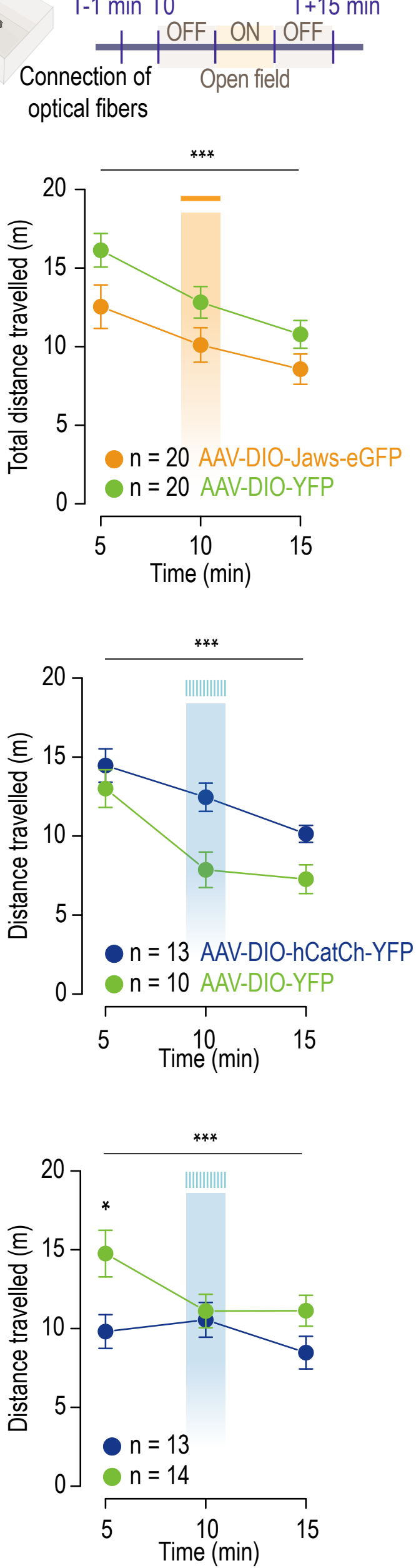

Figure S4.3 


\section{Figure S4.4}

539 (A) (Left) Representative immunohistofluorescence analysis of VTA slices for Jaws expression (GFP:

540 green labeling, TH: red labeling). (Right) Optical fibers were implanted in the basolateral Amg (BLA,

541 bregma -1.61; lateral 3.18; ventral $4.7 \mathrm{~mm}$ ) of wild-type (WT) mice injected either with AAV-CAG-Jaws-

542 GFP (orange dots represent the location of one hemisphere fiber tip that was verified, $n=7$ ) or with AAV-

543 CAG-GFP control (green dots, $n=3$ ) into the VTA. (B) Mice implanted in the BLA were tested for any

544 difference in locomotor activity between groups in the open field (OF). The test lasted 15 minutes and

545 consisted of a 5-minute period of photostimulation (continuous at $520 \mathrm{~nm}$ ) in between two non-stimulation

546 periods (OFF-ON-OFF). During both OFF- and ON-periods, the groups did not present any statistically

547 significant difference in the distance traveled in the OF (two-way RM ANOVA main effect epoch $F_{(2,44)}=$

$5485.89,{ }^{* *} p=0.005$, no opsin or interaction effect, post-hoc Student's t-test with Bonferroni corrections, $p$ >

549 0.05). (C) Inhibiting BLA axon terminals by photostimulation of inhibitory opsin Jaws during the EOM task

550 induced a decrease in the time spent by the mice in the open arms compared to the control group (two-

551 way RM ANOVA, epoch $x$ opsin interaction $F_{(2,42)}=3.44$, * $p=0.04$, post-hoc Student's t-test $p$ (ON Jaws

552 vs GFP) $=0.056$; post-hoc Student's t-test with Bonferroni corrections Jaws * $\mathrm{p}$ ( 5 minutes vs 10 minutes)

$553=0.01 ;{ }^{*} p(10$ minutes vs 15 minutes $\left.)=0.005\right)$. (D) (Left) Representative immunohistofluorescence

554 analysis of VTA slices for Jaws expression (GFP: green labeling, TH: red labeling). (Right) Optical fibers

555 were bilaterally implanted in the central amygdala (CeA) of another group of WT mice injected with either

556 AAV-CAG-Jaws-GFP or with AAV-CAG-GFP control into the VTA. Orange dots and green dots represent

557 the location of one hemisphere fiber tip that was verified in animals expressing Jaws $(n=7)$ and in control

558 animals expressing GFP ( $n=7$ ), respectively. (E) Inhibiting CeA terminals by photostimulation did not

559 produce any statistically significant difference in locomotor activity in the OF test between the Jaws- and

560 GFP-expressing groups (two-way RM ANOVA main effect epoch $F_{(2,32)}=23.11$, ${ }^{* * *} p<0.001$, epoch $x$

561 opsin interaction $F_{(2,32)}=3.8,{ }^{*} p=0.03$, no opsin effect, post-hoc Student's t-test with Bonferroni

562 corrections, $p>0.05)$. (F) The two groups did not display any difference in time spend in the open arm of

563 the EOM test either (two-way RM ANOVA, epoch $x$ opsin interaction $F_{(2,32)}=3.67,{ }^{*} p=0.04$, post-hoc

564 Student's t-test with Bonferroni corrections $p>0.05$ ).

565 IF: interfascicular nucleus ; IPN: interpeduncular nucleus ; ml: medial lemniscus ; SNc: substantia nigra

566 pars compacta 
A AAV-GFP

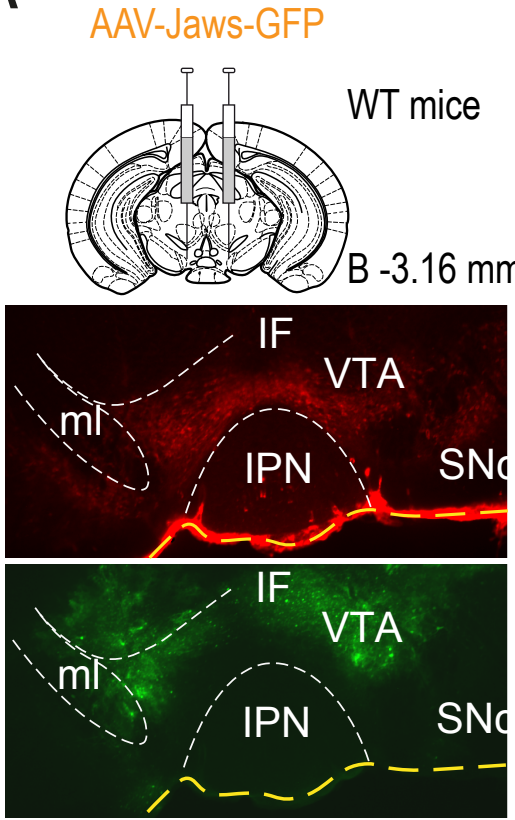

D AAV-GFP

AAV-Jaws-GFP
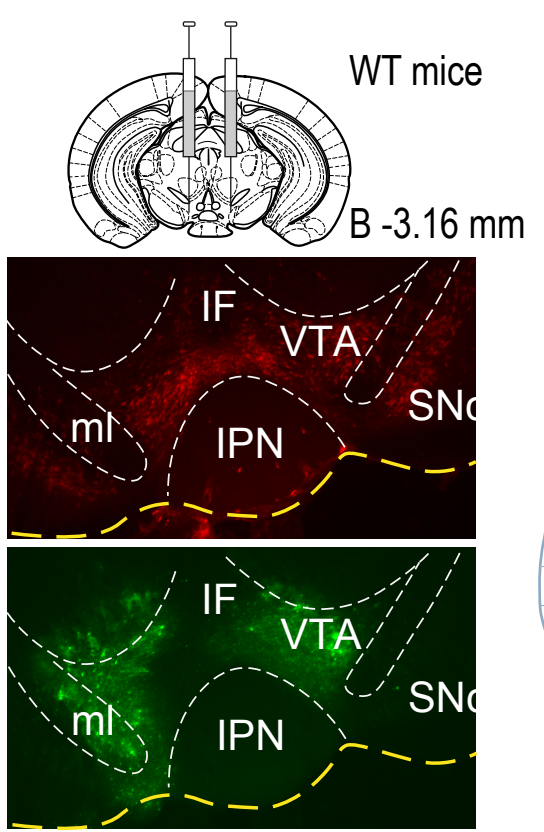

Optical fibers BLA
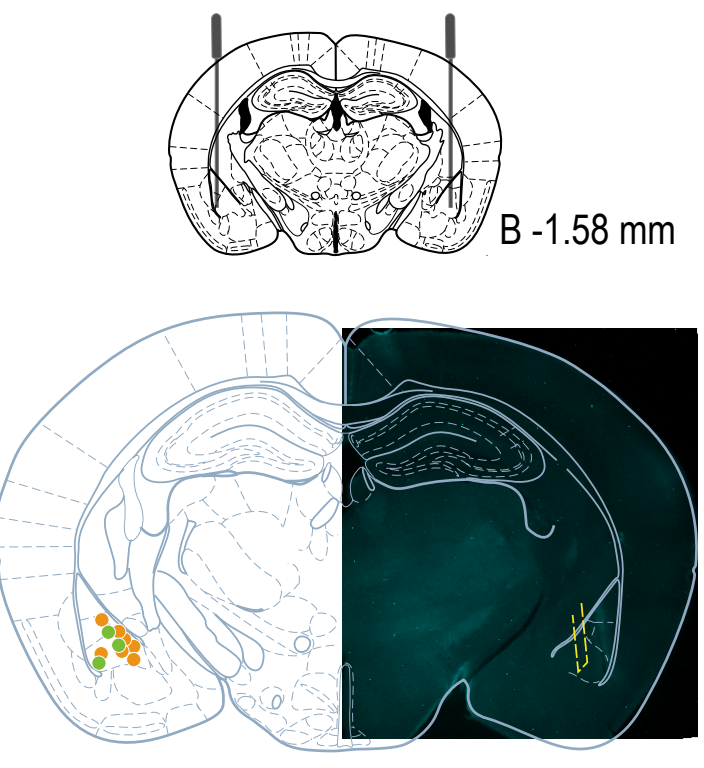

Optical fibers CeA
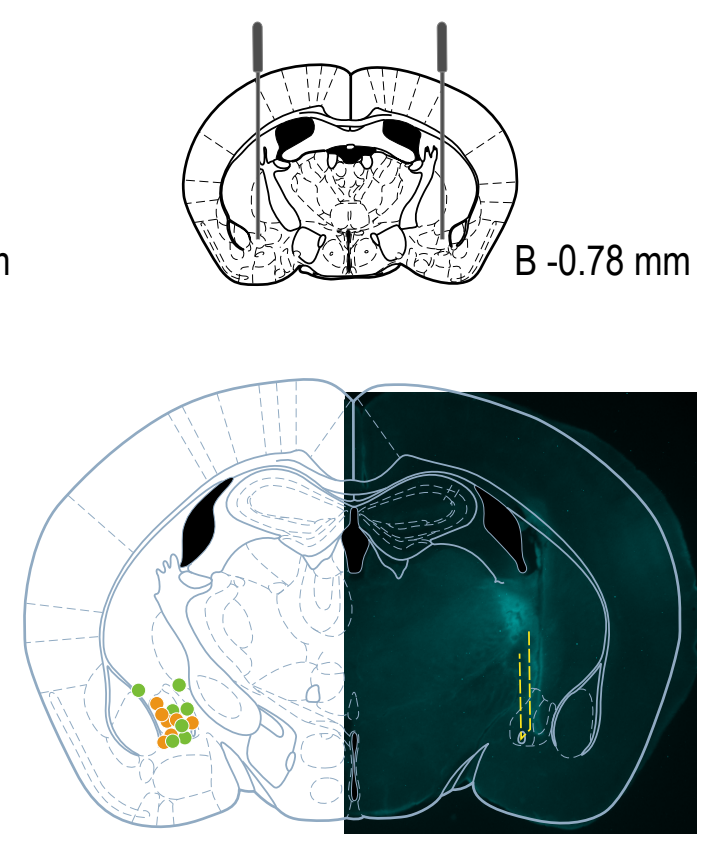

B

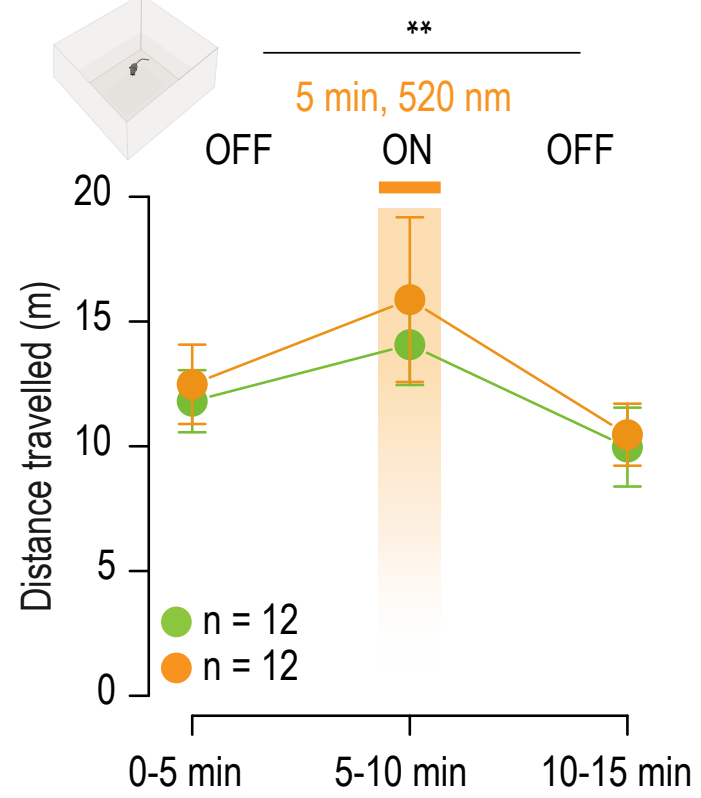

E

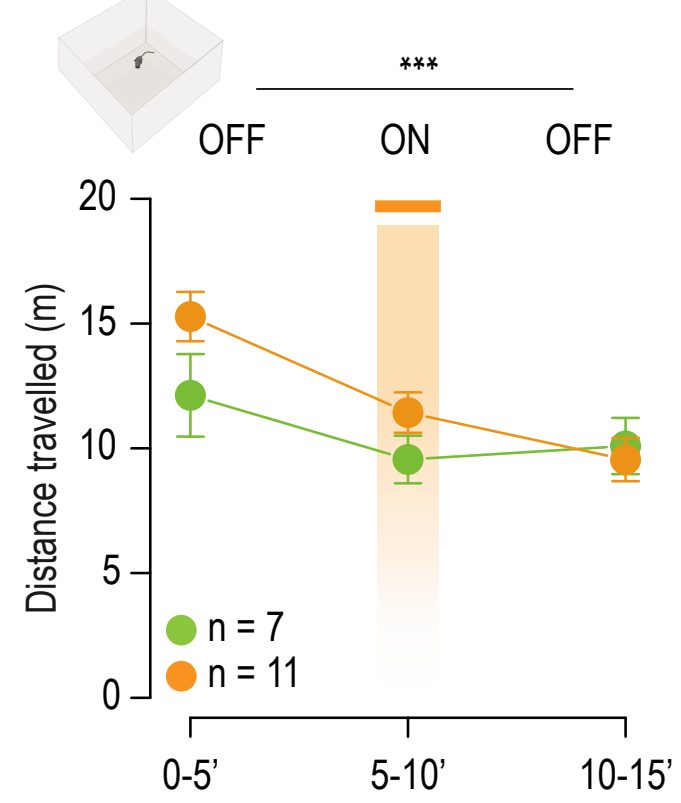

C
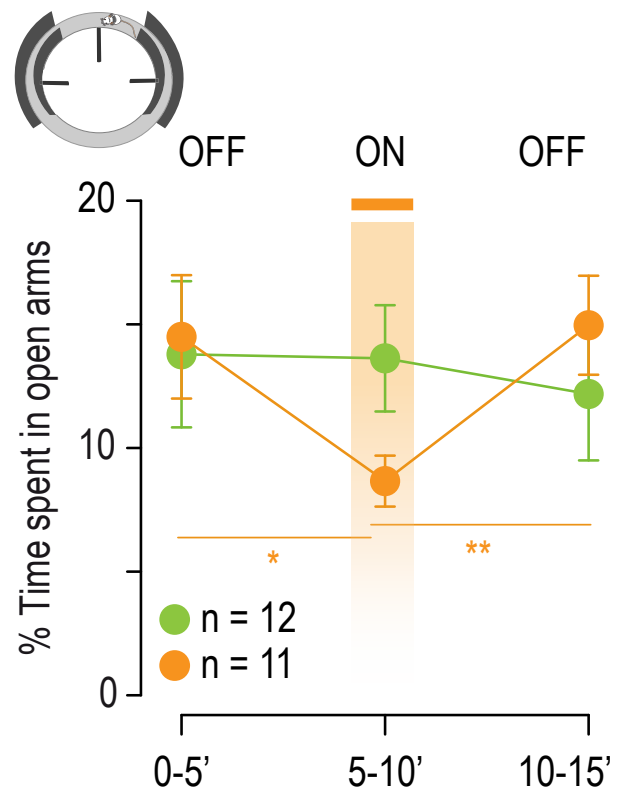

$\mathrm{F}$

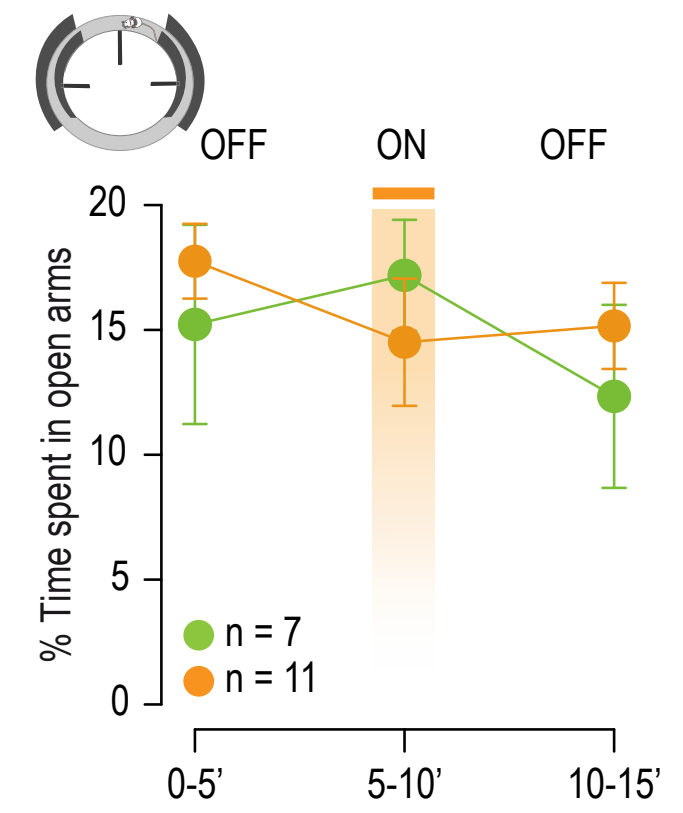

Figure S4.4 


\section{Materials and methods}

\section{Animals}

Wild-type C57BI/6Rj (Janvier Labs, France), ACNB2 KO ( $\beta 2^{---}$) and DATiCRE (DAT-Cre) male mice, weighing 25-35 grams, were used for all experiments. $\beta 2^{--}$mice were generated using standard homologous recombination procedures. Founders were backcrossed onto a C57BL/6J background for a least 20 generations and bred on site. DATiCRE mice were provided by François Tronche's team (IBPS Paris, France). They were bred on site and genotyped as described (Turiault et al., 2007).

Mice were kept in an animal facility where temperature $\left(20 \pm 1^{\circ} \mathrm{C}\right)$ and humidity were automatically monitored, and a circadian light-dark cycle of 12/12 hours was maintained. All experiments were performed on 8-to-16-week-old mice. All experiments were performed in accordance with the recommendations for animal experiments issued by the European Commission directives 219/1990, 220/1990 and 2010/63, and approved by Sorbonne University.

\section{AAV production}

AAV vectors were produced as previously described (Khabou et al., 2018) using the co-transfection method, and purified by iodixanol gradient ultracentrifugation (Choi et al., 2007). AAV vector stocks were titrated by quantitative PCR (qPCR) (Aurnhammer et al., 2012) using SYBR Green (Thermo Fischer Scientific). Lentiviruses were prepared as previously described (Maskos et al., 2005; Tolu et al., 2013), with a titer of either $380 \mathrm{ng}$ of p24 protein per $\mu \mathrm{l}$ or $764 \mathrm{ng} / \mu \mathrm{l}$ for the AChR $\beta 2$-expressing vector, and 150 ng of p24 protein per $\mu$ l or $361 \mathrm{mg}$ per $2 \mu \mathrm{l}$ for GFP-expressing vector.

\section{Drugs}

The solution of nicotine (Nic) used for all experiments is a nicotine hydrogen tartrate salt (Sigma-Aldrich, USA). For juxtacellular recordings, we performed an intravenous injection of nicotine at a dose of $30 \mu \mathrm{g} / \mathrm{kg}$ (4.16 mg/kg, free base) or saline water $\left(\mathrm{H}_{2} \mathrm{O}\right.$ with $\left.0.9 \% \mathrm{NaCl}\right)$. For the behavioral test, in elevated Omaze (EOM) or open-field (OF), mice were injected intra-peritoneally with nicotine at $0.5 \mathrm{mg} / \mathrm{kg} 1$-minute prior to the test. For intra-cranial (IC) experiments in EOM, saline solution or 100ng of Nic tartrate were infused in $100 \mathrm{nl} /$ /injection over 1 minute before the beginning of the test. All solutions were prepared in the laboratory.

\section{Retrobeads injection}

Green fluorescent retrobeads (RB) tracers (LumaFluor Inc., Naples, FL) were injected (200 nl per site, $0.1 \mu \mathrm{l} / \mathrm{min}$ ) in wild-type (WT) animals either in the NAc (NAc lateral shell NAcLatSh : bregma $+1.45 \mathrm{~mm}$; lateral $1.75 \mathrm{~mm}$; ventral $4.0 \mathrm{~mm}$; NAc medial shell : bregma $+1.78 \mathrm{~mm}$; lateral $0.45 \mathrm{~mm}$; ventral $4.1 \mathrm{~mm}$ ; NAc core : bregma $1.55 \mathrm{~mm}$; lateral $1.0 \mathrm{~mm}$; ventral $4.0 \mathrm{~mm}$ ) or in the Amg (BLA : bregma - $1.61 \mathrm{~mm}$; lateral $3.18 \mathrm{~mm}$; ventral $4.7 \mathrm{~mm}$; CeA : bregma - $0.78 \mathrm{~mm}$; lateral $2.3 \mathrm{~mm}$; ventral $4.8 \mathrm{~mm}$ ) with a $10 \mu \mathrm{l}$ Hamilton syringe (Hamilton) coupled with a polyethylene tubing to a $36 \mathrm{G}$ injection cannula (Phymep). Note that these empirically derived stereotaxic coordinates do not precisely match those given in the mouse brain atlas (Paxinos and Franklin, 2004), which we used as references for the injection-site images. To enable retrograde transport of the RB into the somas of midbrain DA neurons, we waited for an adequate time to perform the electrophysiology experiments, depending on the injection zone: 3 weeks after NAc injection and 2 weeks after Amg injection. 
606

607

608

609

610

611

612

613

614

615

616

617

618

619

620

621

622

623

624

625

626

627

628

629

630

\section{Virus injection and optogenetics experiments}

DAT TCRE mice were anesthetized (Isoflurane 1\%) and IC injection was performed into the VTA (coordinates bregma: + $3.1 \mathrm{~mm}$; lateral: $0.5 \mathrm{~mm}$; ventral: $4.5 \mathrm{~mm}$ ) with $0.5 \mu \mathrm{l}$ of an adeno-associated virus

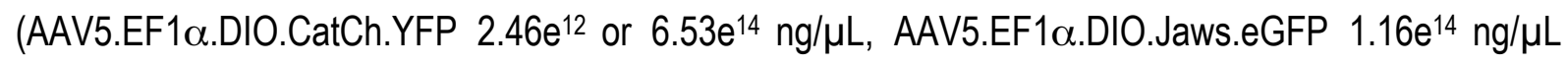
diluted at 1:10, AAV5.EF1 $\alpha$. DIO.YFP $6.89 \mathrm{e}^{14}$ or $9.10 \mathrm{e}^{13} \mathrm{ng} / \mu \mathrm{L}$ ). A double-floxed inverse open reading frame (DIO) allowed restraining the expression of CatCh ( $\mathrm{Ca}^{2+}$ translocating channelrhodopsin) or Jaws (red-shifted cruxhalorhodopsin) to VTA DA neurons. Optical fibers (200 $\mu \mathrm{m}$ core, NA $=0.39$, Thor Labs) coupled to a ferule $(1.25 \mathrm{~mm})$ were implanted bilaterally in the different target sites of the VTA (coordinates for BLA implantation: bregma: - $1.6 \mathrm{~mm}$; lateral: $3.18 \mathrm{~mm}$; ventral: $4.5 \mathrm{~mm}$ ) (coordinates for NAc LatSh implantation: bregma: $+1.5 \mathrm{~mm}$; lateral: $1.55 \mathrm{~mm}$; ventral: $3.95 \mathrm{~mm}$ ), and fixed to the skull with dental cement (SuperBond, Sun medical). An ultra-high-power LED coupled to a patch cord (500 $\mu \mathrm{m}$ core, NA $=0.5$, Prizmatix) was used for optical stimulation (output intensity of $10 \mathrm{~mW}$ ). Optical stimulation was delivered at a frequency of $10 \mathrm{~Hz}, 5 \mathrm{~ms}$-pulse at $470 \mathrm{~nm}$ (Prizmatix LED) for activating stimulation, and with a continuous stimulation at $520 \mathrm{~nm}$ (Prizmatix LED) for inhibiting stimulation.

Optogenetic experiment performed on WT mice were conducted in the same manner as described above. Viruses were bilaterally injected into the VTA at the same coordinates as previously.

The first experiments were conducted on the animals at least 4 weeks after virus injection, to allow the construct to expressed by target cells. The optical stimulation cable was plugged onto the ferule during all experimental sessions to prepare the animals and control for latent experimental effects.

To perform non-specific optogenetic stimulation of different subnuclei of the amygdala (Amg), either

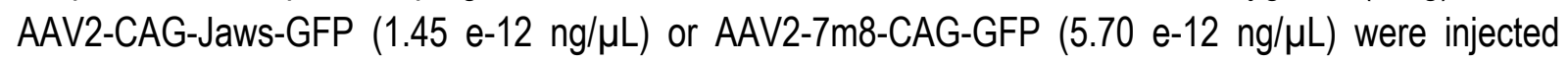
bilaterally into the VTA of distinct groups of wild-type (WT) mice as described in Methods. Bilateral optical fibers were implanted in those mice either in the basolateral amygdala (BLA, bregma: $-1.6 \mathrm{~mm}$; lateral: $3.18 \mathrm{~mm}$; ventral: $4.5 \mathrm{~mm}$ ) or in the central amygdala (CeA, bregma: $-0.78 \mathrm{~mm}$; lateral: $2.3 \mathrm{~mm}$; ventral: $4.8 \mathrm{~mm})$.

\section{Intracranial infusion}

Bilateral guide cannulae (Bilaney) were implanted in the VTA (bregma: + $3.1 \mathrm{~mm}$; lateral: $0.5 \mathrm{~mm}$; ventral: $4.3 \mathrm{~mm}$ ) of mice under anaesthesia (Isoflurane 3\% induction and 1.5\% maintenance) in order to allow local infusion of drugs prior to the EOM experiment. Before each experiment session, a double injection cannulae (4.5 mm length, $1 \mathrm{~mm}$ interval) was inserted into the implanted bilateral guide cannulae (length under pedestal $4.0 \mathrm{~mm}$ ), $0.5 \mathrm{~mm}$ beyond the tip of the guide cannula. The injection cannula was connected to a multi-syringe pump (Univentor) that allowed saline or nicotine injection over $1 \mathrm{~min}$ (100 $\mathrm{ng}$ in $100 \mathrm{nl} /$ injection).

\section{In vivo electrophysiology}

Mice were deeply anaesthetized with chloral hydrate (8\%), $400 \mathrm{mg} / \mathrm{kg}$ I.P., supplemented as required to maintain optimal anesthesia throughout the experiment. The scalp was opened and a hole was drilled in the skull above the location of the VTA. Intravenous administration of drugs was carried out through a catheter into the saphenous vein of the animal. Extracellular recording electrodes were constructed from $1.5 \mathrm{~mm}$ O.D. / $1.17 \mathrm{~mm}$ I.D. borosilicate glass tubing (Harvard Apparatus) using a vertical electrode puller (Narishige). The tip was broken straight and clean under microscopic control to obtain a diameter of about 
$6461 \mu \mathrm{m}$. The electrodes were filled with a $0.5 \% \mathrm{NaCl}$ solution containing $1.5 \%$ of neurobiotin ${ }^{\circledR}$ tracer 647 (VECTOR laboratories) yielding impedances of 6-9 M 2 . Electrical signals were amplified by a high648 impedance amplifier (Axon Instruments) and monitored audibly through an audio monitor (A.M. Systems 649 Inc.). The signal was digitized, sampled at $25 \mathrm{kHz}$, and recorded on a computer using Spike2 software 650 (Cambridge Electronic Design) for later analysis. The electrophysiological activity was sampled in the 651 central region of the VTA (coordinates: between 3.1 to $4 \mathrm{~mm}$ posterior to bregma, 0.3 to $0.7 \mathrm{~mm}$ lateral 652 to midline, and 4 to $4.8 \mathrm{~mm}$ below brain surface). Individual electrode tracks were separated from one 653 another by at least $0.1 \mathrm{~mm}$ in the horizontal plane. Spontaneously active DA neurons were identified 654 based on previously established electrophysiological criteria (Grace and Bunney, 1984b; 1984a; Ungless 655 and Grace, 2012).

656 After recording, nicotine-responsive cells were labelled by electroporation of their membrane. To do so, 657 successive currents squares were applied until the membrane breaks in order to fill their soma with 658 neurobiotin contained into the glass pipet (Pinault 1996). To be able to establish correspondence between 659 neurons responses and their localization in the VTA, we labeled one type of response per mouse: solely 660 activated neurons or solely inhibited neurons, with a limited number of cells per brain (1 to 4 neurons 661 maximum, 2 by hemisphere), always with the same concern of localization of neurons in the VTA.

\section{Ex vivo patch-clamp recordings}

663 For a functional verification of CatCh or Jaws expression, the same virus as described above was injected 664 into 7-9 week-old male DATiCRE mice. For the characterization of NAc-projecting and Amg-projecting 665 neurons, green retrobead tracers (Lumafluor) were injected into 7-9 weeks old male WT mice. After 4 666 weeks (for DAT-Cre) or 2 weeks (for WT), mice were deeply anesthetized by an intraperitoneal injection 667 of a mix of ketamine (150 mg/kg Imalgene® 1000, Merial) and xylazine (60 mg/kg, Rompun® $2 \%$, Bayer). 668 Coronal midbrain sections $(250 \mu \mathrm{m})$ were sliced with a Compresstome (VF-200, Precisionary 669 Instruments) after intracardial perfusion of cold $\left(4^{\circ} \mathrm{C}\right)$ sucrose-based artificial cerebrospinal fluid (SB670 aCSF) containing (in mM): $125 \mathrm{NaCl}, 2.5 \mathrm{KCl}, 1.25 \mathrm{NaH}_{2} \mathrm{PO}_{4}, 5.9 \mathrm{MgCl}_{2}, 26 \mathrm{NaHCO}_{3}, 25$ sucrose, 2.5

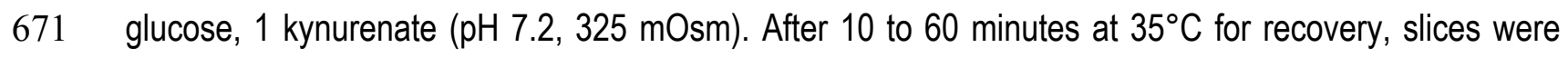
672 transferred into oxygenated aCSF containing (in $\mathrm{mM}$ ): $125 \mathrm{NaCl}, 2.5 \mathrm{KCl}, 1.25 \mathrm{NaH}_{2} \mathrm{PO}_{4}, 2 \mathrm{CaCl}_{2}, 1$ $673 \mathrm{MgCl}_{2}, 26 \mathrm{NaHCO}_{3}, 15$ sucrose, 10 glucose (pH 7.2, $325 \mathrm{mOsm}$ ) at room temperature for the rest of the 674 day, and individually transferred to a recording chamber continuously perfused at $2 \mathrm{~mL} /$ minute with 675 oxygenated aCSF. Patch pipettes (4-8 M $\Omega$ ) were pulled from thin wall borosilicate glass (G150TF-3, 676 Warner Instruments) with a micropipette puller (P-87, Sutter Instruments, Novato, CA) and filled with a 677 potassium gluconate-based intracellular solution containing either (in $\mathrm{mM}$ ): $116 \mathrm{~K}$-gluconate, $20 \mathrm{HEPES}$, $6780.5 \mathrm{EGTA}, 6 \mathrm{KCl}, 2 \mathrm{NaCl}, 4 \mathrm{ATP}, 0.3 \mathrm{GTP}$, and biocytin $2 \mathrm{mg} / \mathrm{mL}$ (pH adjusted to 7.2). Neurons were 679 visualized using an upright microscope coupled with a Dodt contrast lens, and illuminated with a white 680 light source (Scientifica). A $460 \mathrm{~nm}$ LED ( $\mathrm{pE}-2$, Cooled) was used for visualizing GFP, YFP- or RB positive 681 cells (using a bandpass filter cube, AHF). Whole-cell recordings were performed using a patch-clamp 682 amplifier (Axoclamp 200B, Molecular Devices) connected to a Digidata (1550 LowNoise acquisition 683 system, Molecular Devices). Signals were low-pass filtered (Bessel, $2 \mathrm{kHz}$ ) and collected at $10 \mathrm{kHz}$ using 684 the data acquisition software pClamp 10.5 (Molecular Devices). Optical stimulation was applied through 685 the microscope with two LEDs (460 nm and $525 \mathrm{~nm}, \mathrm{pE}-2$, CoolLED). To characterize CatCh expression, 686 a $1 \mathrm{~s}$ continuous photostimulation was used to evoke currents in voltage-clamp mode $(-60 \mathrm{mV})$, and a 10 $687 \mathrm{~Hz}-5 \mathrm{~ms} /$ pulse photostimulation was used to drive neuronal firing in current-clamp mode. Regarding 688 Jaws expression, continuous photostimulation $(20 \mathrm{sec})$ was used in current-clamp $(-60 \mathrm{mV})$. To record 
nicotinic currents from RB+ DA neurons of the VTA, local puffs (500 ms) of nicotine tartrate (100 $\mu \mathrm{M}$ in aCSF) were applied with a glass pipette (2-3 $\mu \mathrm{m}$ diameter) positioned 20 to $30 \mu \mathrm{m}$ away from the soma and connected to a picospritzer (World Precision Instruments, adjusted to $\sim 2 \mathrm{psi}$ ). All electrophysiological recordings were extracted using Clampfit (Molecular Devices) and analyzed with $\mathrm{R}$.

\section{Immunostaining}

After euthanasia, brains were rapidly removed and fixed in $4 \%$ paraformaldehyde. After a period of at least three days of fixation at $4^{\circ} \mathrm{C}$, serial $60-\mu \mathrm{m}$ sections were cut from the midbrain with a vibratome. Immunostaining experiments were performed as follows: free-floating VTA brain sections were incubated for 1 hour at $4^{\circ} \mathrm{C}$ in a blocking solution of phosphate-buffered saline (PBS) containing $3 \%$ bovine serum albumin (BSA, Sigma; A4503) (vol/vol) and 0.2\% Triton X-100 (vol/vol), and then incubated overnight at $4{ }^{\circ} \mathrm{C}$ with a mouse anti-tyrosine hydroxylase antibody (anti-TH, Sigma, T1299) and a chicken anti-eYFP antibody (Life technologies Molecular Probes, A-6455), both at 1:500 dilution, in PBS containing 1.5\% BSA and $0.2 \%$ Triton $X-100$. The following day, sections were rinsed with PBS, and then incubated for 3 hours at $22-25{ }^{\circ} \mathrm{C}$ with Cy3-conjugated anti-mouse and Alexa488-conjugated anti-chicken secondary antibodies (Jackson ImmunoResearch, 715-165-150 and 711-225-152) at 1:500 and 1:1000 dilution in a solution of $1.5 \%$ BSA in PBS, respectively. After three rinses in PBS, slices were wet-mounted using Prolong Gold Antifade Reagent (Invitrogen, P36930). Microscopy was carried out with a fluorescent microscope, and images captured using a camera and analyzed with ImageJ.

In the case of electrophysiological recordings, the recorded neurons were identified by immunohistofluorescence as described above, with the addition of 1:200 AMCA-conjugated streptavidin (Jackson ImmunoResearch) in the solution. Immunoreactivity for both $\mathrm{TH}$ and neurobiotin (NB) allowed us to confirm the neurochemical phenotype of DA neurons in the VTA $(T H+N B+)$.

In the case of optogenetic experiments on DATICRE mice, identification of the transfected neurons by immunohistofluorescence was performed as described above, with the addition of chicken-anti-eYFP primary antibody (1:500, ab13970, Abcam) in the solution. A goat-anti-chicken AlexaFluor 488 (1:500, Life Technologies) was then used as secondary antibody. Immunoreactivity for TH, YFP and neurobiotin/biocytin allowed us to confirm the neurochemical phenotype of DA neurons in the VTA (TH+ $\mathrm{NB}+$ ) and the transfection success (YFP+).

\section{Images acquisition}

All immunohistochemical slices were imaged by acquisition on a Leica DMR epi-fluorescent microscope, under identical conditions of magnification, illumination and exposure (using photometrics coolsnap camera). Images were captured in gray level using MetaView software (Universal Imaging Corporation, Ropper Scientific, France) and colored post-acquisition on ImageJ software.

\section{Behavioral data acquisition}

All behavioral tests were conducted during the light period of the animal cycle (between 1:00 and 7:00PM). Experimental data were acquired using Anymaze software, coupled with 2D video camera. 
The EOM apparatus consists of two open (stressful) and two enclosed (protecting) elevated arms that together form a zero or circle (diameter of $50 \mathrm{~cm}$, height of $58 \mathrm{~cm}, 10 \mathrm{~cm}$-wide circular platform). Time spent in exploring enclosed versus open arms indicates then the anxiety level of the animal. The first EOM experiment assess the effect of acute Nic injection $(0.5 \mathrm{mg} / \mathrm{kg})$ on WT animals. The test lasts 10 minutes. Animals are injected 1-min prior the test and then put in the EOM for 9 min. For intra-cranial infusion of Nic in the second experiment of EOM, animals received Nic (100ng/infusion) over 1-min prior the test. It lasted for 9 min as described above. stimulation and non-stimulation (OFF-ON-OFF). When nicotine was injected in mice, the EOM test lasted for $9 \mathrm{~min}$ with the same protocol as described above.

Online place preference (OPP) test

The OPP protocols were performed in a Y-maze apparatus (Imetronic, Pessac, France), using only two arms of the Y-maze as two distinct compartments (the third arm was closed by a door and not available to the animal). The chamber in between is an equilateral triangle (side of $11 \mathrm{~cm}$ ) used as a neutral compartment, where the animal was never photo-stimulated. Each arm of the maze measured $25 \mathrm{~cm} \times$ $12 \mathrm{~cm}$. The first arm displayed black and white stripes with smooth walls and floor, whereas the other arm displayed uniform-gray rough walls and floor. Choices of the compartment where the animals will be stimulated were counterbalanced across animals in the same test and YFP-control groups. The OPP test consisted of a 20 minute-session where animals can freely navigate between the compartments but were photo-stimulated only in one of the two compartments. Implanted bilateral fibers were connected with a bilateral fiber (diameter of $400 \mu \mathrm{m}, \mathrm{NA}=0.39$, Thorlabs) attached to a rotor connecting the $470 \mathrm{~nm}$ - or $520 \mathrm{~nm}$-LED (Prizmatix) with a fiber of diameter $500 \mu \mathrm{m}$ and NA $=0.5$ (Thorlabs). LED output was controlled using a Master-8 pulse stimulator (A.M.P.I., Jerus) which delivered a discontinuous stimulation of 5 -ms light flashes at $10 \mathrm{~Hz}$ frequency and $470 \mathrm{~nm}$ wavelength (for CatCh experiments), or a continuous stimulation at $520 \mathrm{~nm}$ (for Jaws experiments). Naive

751 mice were connected and placed at the center of the neutral compartment before starting the recording.

753 The OF is a square enclosure of $50 \mathrm{~cm} \times 50 \mathrm{~cm}$ where animals can move freely. Animal displacements 754 were quantified by comparing the time spent in the center versus the periphery of the square. When nicotine was injected to WT mice in the OF test (IP injection of nicotine tartrate at $0.5 \mathrm{mg} / \mathrm{kg}, 0.1 \mathrm{~mL} / 10$

$756 \mathrm{~g}, 1$ minute prior to the test), animals were placed in the center of the OF for a 9-minute test duration, 757 freely moving inside the enclosure. Regarding the optogenetic experiments conducted in the OF, animals 758 were placed in the maze for 15 minutes, while alternating between OFF, ON and OFF optical stimulations 759 of 5 -minute periods.

\section{Data Analyses}

761 Measurement of neuronal activity

762 Timestamps of action potentials were extracted in Spike 2 and analyzed using $\mathrm{R}$, a language and 763 environment for statistical computing (Team, 2005, http://www.r-project.org). Spontaneous activity of DA 764 cell firing in vivo was analyzed with respect to the average firing frequency (in $\mathrm{Hz}$ ) and the percentage of 
spikes-within-burst (\%SWB = number of spikes within burst divided by total number of spikes in a given window). Neuronal basal activity was defined on at least five-minute recording.

\section{Classification analysis and machine learning}

\section{To determine whether the spontaneous activity of in vivo-labeled, VTA DA neurons could predict their} nicotine-evoked responses (activation or inhibition), we analyzed 8 variables that characterize the firing patterns: the mean firing frequency, the standard deviation and coefficient of variation of the firing frequency estimated on sliding windows, the percentage of spikes within bursts, the bursting frequency, the mean number of spikes in a burst, the fraction of time spent in bursting activity, and the burst length. Firing frequency and burst activity are quantified as described in the materials and methods section. Principal component analysis (PCA) and hierarchical clustering on principle components (HCPC) were conducted using R package FactoMineR. PCA and clustering distinguished 4 clusters that did not match with the nicotine-evoked responses. We then used the $\mathrm{R}$ package RandomForest to test for a prediction of nicotine-evoked responses from spontaneous cell activity. We determined that 5000 was the optimal number of trees in the forest and that 2 was the best number of variables randomly sampled as candidates at each split.

\section{Quantification of nicotine responses}

Firing frequency was quantified on overlapping 60 -second windows shifted by 15 seconds time steps. For each neuron, firing frequency was rescaled as a percentage of its baseline value averaged during 3 minutes before nicotine injection. The responses to nicotine were thus presented as a percentage of variation from baseline (mean \pm S.E.M.). The effect of nicotine was assessed by comparison of the maximum of firing frequency variation induced by nicotine and saline injection. For activated (respectively inhibited) neurons, the maximal (respectively minimal) value of the firing frequency was measured within the response period (3 minutes) that followed nicotine or saline injection. The results were presented as mean \pm S.E.M. of the difference of maximum variation after nicotine or saline.

\section{Subpopulations of VTA DA neurons in response to nicotine}

Subpopulations of DA neurons were automatically classified using variation of firing frequency and the following routine: considering the maximal variation from a short baseline ( 3 minutes before injection), within 3 minutes after injection, neurons displaying an increase in firing frequency were defined as "activated", and neurons displaying a decrease in firing frequency were defined as "inhibited" (up or below zero in the cumulative distribution of responses to nicotine). In $\beta 2$ \% mice, VTA DA neurons did not show a clear change of their firing rate after nicotine injection (i.e., less than $5 \%$ of variation from baseline), and therefore were considered as a unique population to represent the mean response variation in Figure $3 \mathrm{~B}$.

\section{Juxtacellular labeling}

A total number of 245 neurons have been recorded and labeled for Figure 1. Those 245 neurons were used in Figure 1B-D. Among them, 101 neurons were shown in Figure 2B and E, containing 49 neurons labeled in NAc-RB injected mice and 52 in Amg-RB injected mice. The locations of the labeled neurons were manually placed on sections of the Paxinos atlas georeferenced in a $2 \mathrm{D}$ grid using adobe illustrator rules. The medio-lateral and dorso-ventral coordinates of the location of each neuron were extracted from the grid pattern and the antero-posterior coordinates were estimated from the section of the Paxinos atlas 
805

806

807

808

809

810

811

812

813

814

815

816

817

818

819

820

821

822

823

824

825

826

827

828

829

830

831

832

833

834

835

836

837

838

839

840

841

842

843

on which the neurons were placed. These three coordinates were used to make density histograms of location for nicotine-activated and nicotine-inhibited DA neurons or NAc-projecting and Amg-projecting DA neurons.

\section{Behavioral experiments}

The raw data for behavioral experiments were acquired as the time spent by animals in the different zones of the environments. The animals were detected in their body center by a USB camera connected to Anymaze software.

\section{Statistical analyses}

All statistical analyses were done using the R software with home-made routines. Results are plotted as a mean \pm S.E.M. The total number $(n)$ of observations in each group and the statistics used are indicated in the figures directly or in the figure legends. Classically comparisons between means were performed using parametric tests as Student's t-test, or two-way ANOVA for comparing two groups when parameters followed a normal distribution (Shapiro-Wilk normality test $p>0.05$ ), or Wilcoxon non-parametric test as when the distribution was skewed. Bonferroni post-hoc analysis was applied, when necessary, to compare means. $P>0.05$ was considered not to be statistically significant. Statistical significance was set at $p<0.05\left(^{*}\right), p<0.01\left(^{* *}\right)$, or $p<0.001\left(^{* * *}\right)$.

Figure 1: Kolmogorov-Smirnov test was used to compare the responses of VTA DA neurons to saline or nicotine injection. Wilcoxon tests were used to demonstrate a significant increase or decrease of firing frequency induced by nicotine injection compared to saline injection (B). Wilcoxon test was used to compared coordinates of nicotine-inhibited and nicotine-activated recorded neurons (C).

Figure 3: For behavior (A-C), over time effect of nicotine or saline injection (IP and IC) on the time spent by the mice in the open arms of the O-maze was first tested with one-way repeated measures ANOVA for each group of mice (shown in Figure S3.1B-D-G). Two-way repeated measures ANOVA (time/treatment or time/genotype) were used to compare the difference between the groups. In case of significant interaction effect between factors, Wilcoxon or Student's t-test with Bonferroni corrections were used for intra- and inter-group post-hoc analysis (as indicated in the figure). For electrophysiology (B), Kolmogorov-Smirnov test was used to compare responses to nicotine of DA neurons in $\beta 2^{-/-}$mice and $\beta 2^{-}$ $1-V e c$ mice. Wilcoxon tests with Bonferroni corrections are used to demonstrate a significant increase or decrease of firing frequency induced by IV nicotine injection in $\beta 2 \mathrm{Vec}$ mice compared to saline and nicotine injections in $\beta 2^{--}$mice.

Figure 4: For O-maze experiments (A-C), effect of light was first tested with one-way repeated measures ANOVA for each group of mice (shown in Figure S4.3A-B-C). Two-way repeated measures ANOVA (epoch/opsin) were used to compare the difference between the groups. In case of significant interaction effect between factors, Wilcoxon or Student's t-test with Bonferroni corrections were used for intra- and inter-group post-hoc analysis (as indicated in the figure). For O-maze experiment under nicotine (D), over time effect of nicotine injection on the time spent by the mice in the open arms of the 0-maze was first tested with one-way repeated measures ANOVA for each group. Two-way repeated measures ANOVA (epoch/opsin) were used to compare the difference between the groups. In case of significant interaction effect between factor, Wilcoxon or Student's t-test with Bonferroni corrections were used for intra- and 
844 inter- group post-hoc analysis (as indicated in the figure). For OPP experiments (E), preference score 845 between group were compared with Student's t-test.

846 Figure S2.1: Two-way repeated measures ANOVA (current, phenotype) is used to compare neuronal 847 excitability (Fig. S2.1D). Wilcoxon test is used to compared coordinates of nicotine-inhibited and nicotine848 activated recorded neurons (Fig. S2.1F).

849 Figure S2.2: Wilcoxon test is used to compare nicotine-evoked currents (Fig. S2.2F).

850 Figure S3: Two-way repeated measures ANOVA (time, treatment) is used to compare the distance 851 traveled by mice in the open field (OF) after nicotine or saline injection (Fig. S3A). One-way repeated 852 measures ANOVA is used to test the overtime effect of saline or nicotine intraperitoneal injection, or 853 intracranial infusion, or the time spent by mice in the open arms of the elevated O-maze (EOM) (Fig. S3B, $854 \mathrm{D}, \mathrm{G}$ ). Two-way repeated measures ANOVA (time, genotype) is used to compare the time spent in the 855 open arms of the EOM after nicotine injection between groups (Fig. S3F). In case of a significant 856 interaction effect between factors, Wilcoxon or Student's t-test with Bonferroni corrections is used for 857 intra- and inter-group post-hoc analysis.

858 Figure S4.3: For anxiety, one-way repeated measures ANOVA is used to test the effect of light on the 859 time spent in the open arms of the EOM (Fig. 4.3A-C). For locomotor activity, two-way repeated measures 860 ANOVA (epoch, opsine) is used to compare the difference of light effect on the distance traveled by the 861 mice between the groups (Fig. 4.3D-F). In case of a significant interaction effect between factors, 862 Wilcoxon or Student's t-test with Bonferroni corrections is used for intra- and inter-group post-hoc analysis 863 (as indicated in the figure).

864 Figure S4.4: O-maze experiments (C-F) and locomotor activity (C-F) are analyzed as previously described 865 for Fig.S4.3. 


\section{References}

Aurnhammer, C., Haase, M., Muether, N., Hausl, M., Rauschhuber, C., Huber, I., Nitschko, H., Busch, U., Sing, A., Ehrhardt, A., et al. (2012). Universal real-time PCR for the detection and quantification of adenoassociated virus serotype 2-derived inverted terminal repeat sequences. Hum Gene Ther Methods 23 , 18-28.

Balerio, G.N., Aso, E., and Maldonado, R. (2006). Role of the cannabinoid system in the effects induced by nicotine on anxiety-like behaviour in mice. Psychopharmacology 184, 504-513.

Beier, K.T., Gao, X.J., Xie, S., DeLoach, K.E., Malenka, R.C., and Luo, L. (2019). Topological Organization of Ventral Tegmental Area Connectivity Revealed by Viral-Genetic Dissection of InputOutput Relations. CellReports 26, 159-167.e6.

Beier, K.T., Steinberg, E.E., DeLoach, K.E., Xie, S., Miyamichi, K., Schwarz, L., Gao, X.J., Kremer, E.J., Malenka, R.C., and Luo, L. (2015). Circuit Architecture of VTA Dopamine Neurons Revealed by Systematic Input-Output Mapping. Cell 162, 622-634.

Brischoux, F., Chakraborty, S., Brierley, D.I., and Ungless, M.A. (2009). Phasic excitation of dopamine neurons in ventral VTA by noxious stimuli. Proc Natl Acad Sci USA 106, 4894-4899.

Changeux, J.-P., Bertrand, D., Corringer, P.J., Dehaene, S., Edelstein, S., Léna, C., Le Novère, N., Marubio, L., Picciotto, M., and Zoli, M. (1998). Brain nicotinic receptors: structure and regulation, role in learning and reinforcement. Brain Res Brain Res Rev 26, 198-216.

Changeux, J.-P. (2010). Nicotine addiction and nicotinic receptors: lessons from genetically modified mice. Nat Rev Neurosci 11, 389-401.

Choi, V.W., Asokan, A., Haberman, R.A., and Samulski, R.J. (2007). Production of recombinant adenoassociated viral vectors. Curr Protoc Hum Genet Chapter 12, Unit12.9-12.9.21.

Chuong, A.S., Miri, M.L., Busskamp, V., Matthews, G.A.C., Acker, L.C., Sørensen, A.T., Young, A., Klapoetke, N.C., Henninger, M.A., Kodandaramaiah, S.B., et al. (2014). Noninvasive optical inhibition with a red-shifted microbial rhodopsin. (Nature Publishing Group).

de Jong, J.W., Afjei, S.A., Pollak Dorocic, I., Peck, J.R., Liu, C., Kim, C.K., Tian, L., Deisseroth, K., and Lammel, S. (2019). A Neural Circuit Mechanism for Encoding Aversive Stimuli in the Mesolimbic Dopamine System. Neuron 101, 133-151.e137.

Di Chiara, G., and Imperato, A. (1988). Drugs abused by humans preferentially increase synaptic dopamine concentrations in the mesolimbic system of freely moving rats. Proc Natl Acad Sci USA 85, 5274-5278.

Doyon, W.M., Dong, Y., Ostroumov, A., Thomas, A.M., Zhang, T.A., and Dani, J.A. (2013). Nicotine Decreases Ethanol-Induced Dopamine Signaling and Increases Self-Administrationvia Stress Hormones. Neuron 1-11.

Durand-de Cuttoli, R., Mondoloni, S., Marti, F., Lemoine, D., Nguyen, C., Naudé, J., d'Izarny-Gargas, T., Pons, S., Maskos, U., Trauner, D., et al. (2018). Manipulating midbrain dopamine neurons and rewardrelated behaviors with light-controllable nicotinic acetylcholine receptors. eLife 7, 15991. 
903

904

905

906

907

908

909

910

911

912

913

914

915

916

917

918

919

920

921

922

923

924

925

926

927

928

929

930

931

932

933

934

935

936

937

938

939

Eddine, R., Valverde, S., Tolu, S., Dautan, D., Hay, A., Morel, C., Cui, Y., Lambolez, B., Venance, L., Marti, F., et al. (2015). A concurrent excitation and inhibition of dopaminergic subpopulations in response to nicotine. Sci. Rep. 5, 8184.

Ford, C.P., Mark, G.P., and Williams, J.T. (2006). Properties and opioid inhibition of mesolimbic dopamine neurons vary according to target location. Journal of Neuroscience 26, 2788-2797.

Fowler, C.D., Lu, Q., Johnson, P.M., Marks, M.J., and Kenny, P.J. (2011). Habenular a5 nicotinic receptor subunit signalling controls nicotine intake. Nature 471, 597-601.

Frahm, S., Ślimak, M.A., Ferrarese, L., Santos-Torres, J., Antolin-Fontes, B., Auer, S., Filkin, S., Pons, S., Fontaine, J.-F., Tsetlin, V., et al. (2011). Aversion to Nicotine Is Regulated by the Balanced Activity of \&beta;4 and \&alpha;5 Nicotinic Receptor Subunits in the Medial Habenula. Neuron 70, 522-535.

Grace, A.A., and Bunney, B.S. (1984a). The control of firing pattern in nigral dopamine neurons: burst firing. J Neurosci 4, 2877-2890.

Grace, A.A., and Bunney, B.S. (1984b). The control of firing pattern in nigral dopamine neurons: single spike firing. J Neurosci 4, 2866-2876.

Grenhoff, J., Aston-Jones, G., and Svensson, T.H. (1986). Nicotinic effects on the firing pattern of midbrain dopamine neurons. Acta Physiol. Scand. 128, 351-358.

Grieder, T.E., George, O., and Tan, H. (2012). Phasic D1 and tonic D2 dopamine receptor signaling double dissociate the motivational effects of acute nicotine and chronic nicotine withdrawal.

Grieder, T.E., Besson, M., Maal-Bared, G., Pons, S., Maskos, U., and van der Kooy, D. (2019). ß2* nAChRs on VTA dopamine and GABA neurons separately mediate nicotine aversion and reward. Proc Natl Acad Sci USA 116, 25968-25973.

Grieder, T.E., Sellings, L.H., Vargas-Perez, H., Ting-A-Kee, R., Siu, E.C., Tyndale, R.F., and van der Kooy, D. (2010). Dopaminergic signaling mediates the motivational response underlying the opponent process to chronic but not acute nicotine. Neuropsychopharmacology 35, 943-954.

Khabou, H., Garita-Hernandez, M., Chaffiol, A., Reichman, S., Jaillard, C., Brazhnikova, E., Bertin, S., Forster, V., Desrosiers, M., Winckler, C., et al. (2018). Noninvasive gene delivery to foveal cones for vision restoration. JCl Insight 3.

Kleinlogel, S., Feldbauer, K., Dempski, R.E., Fotis, H., Wood, P.G., Bamann, C., and Bamberg, E. (2011). Ultra light-sensitive and fast neuronal activation with the $\mathrm{Ca}^{2}+$-permeable channelrhodopsin CatCh. Nature Neuroscience 14, 513-518.

Klink, R., de Kerchove d'Exaerde, A., Zoli, M., and Changeux, J.P. (2001). Molecular and physiological diversity of nicotinic acetylcholine receptors in the midbrain dopaminergic nuclei. J Neurosci 21, 14521463.

Kutlu, M.G., and Gould, T.J. (2015). Nicotine Modulation of Fear Memories and Anxiety: Implications for Learning and Anxiety Disorders. Biochem Pharmacol 1-58.

Lammel, S., Hetzel, A., Häckel, O., Jones, I., Liss, B., and Roeper, J. (2008). Unique properties of mesoprefrontal neurons within a dual mesocorticolimbic dopamine system. Neuron 57, 760-773. 
940 Lammel, S., Lim, B.K., Ran, C., Huang, K.W., Betley, M.J., Tye, K.M., Deisseroth, K., and Malenka,

941 R.C. (2012). Input-specific control of reward and aversion in the ventral tegmental area. Nature 491,

$942 \quad 212-217$.

943 Luscher, C. (2016). The Emergence of a Circuit Model for Addiction. Annu Rev Neurosci 39, annurev-

944 neuro-070815-013920.

945 Mameli-Engvall, M., Evrard, A., Pons, S., Maskos, U., Svensson, T.H., Changeux, J.-P., and Faure, P. 946 (2006). Hierarchical control of dopamine neuron-firing patterns by nicotinic receptors. Neuron 50, $911-$ 947921.

948 Mansvelder, H.D., and McGehee, D.S. (2000). Long-term potentiation of excitatory inputs to brain 949 reward areas by nicotine. Neuron 27, 349-357.

950 Margolis, E.B., Hjelmstad, G.O., Fujita, W., and Fields, H.L. (2014). Direct bidirectional $\mu$-opioid control 951 of midbrain dopamine neurons. Journal of Neuroscience 34, 14707-14716.

952 Margolis, E.B., Mitchell, J.M., Ishikawa, J., Hjelmstad, G.O., and Fields, H.L. (2008). Midbrain dopamine 953 neurons: projection target determines action potential duration and dopamine $\mathrm{D}(2)$ receptor inhibition. 954 Journal of Neuroscience 28, 8908-8913.

955

956

957

958

959

960

961

962

963

964

965

966

967

968

969

970

971

972

973

974

975

976

977

Maskos, U., Molles, B.E., Pons, S., Besson, M., Guiard, B.P., Guilloux, J.-P., Evrard, A., Cazala, P., Cormier, A., Mameli-Engvall, M., et al. (2005). Nicotine reinforcement and cognition restored by targeted expression of nicotinic receptors. Nature 436, 103-107.

Mejias-Aponte, C.A., Ye, C., Bonci, A., Kiyatkin, E.A., and Morales, M. (2015). A Subpopulation of Neurochemically-Identified Ventral Tegmental Area Dopamine Neurons Is Excited by Intravenous Cocaine. J Neurosci 35, 1965-1978.

Mineur, Y.S., Fote, G.M., Blakeman, S., Cahuzac, E.L.M., Newbold, S.A., and Picciotto, M.R. (2016). Multiple Nicotinic Acetylcholine Receptor Subtypes in the Mouse Amygdala Regulate Affective Behaviors and Response to Social Stress. Neuropsychopharmacology 41, 1579-1587.

Molas, S., DeGroot, S.R., Zhao-Shea, R., and Tapper, A.R. (2017). Anxiety and Nicotine Dependence: Emerging Role of the Habenulo-Interpeduncular Axis. Trends Pharmacol Sci 38, 169-180.

Morales, M., and Margolis, E.B. (2017). Ventral tegmental area: cellular heterogeneity, connectivity and behaviour. Nat Rev Neurosci 18, 73-85.

Morel, C., Fattore, L., Pons, S., Hay, Y.A., Marti, F., Lambolez, B., De Biasi, M., Lathrop, M., Fratta, W., Maskos, U., et al. (2014). Nicotine consumption is regulated by a human polymorphism in dopamine neurons. Mol Psychiatry 19, 930-936.

Morel, C., Fernandez, S.P., Pantouli, F., Meye, F.J., Marti, F., Tolu, S., Parnaudeau, S., Marie, H., Tronche, F., Maskos, U., et al. (2017). Nicotinic receptors mediate stress-nicotine detrimental interplay via dopamine cells' activity. Mol Psychiatry 23, 1597-1605.

Pascoli, V., Terrier, J., Hiver, A., and Lüscher, C. (2015). Sufficiency of Mesolimbic Dopamine Neuron Stimulation for the Progression to Addiction. Neuron 88, 1054-1066.

Paxinos, G., and Franklin, K.B.J. (2004). The Mouse Brain in Stereotaxic Coordinates (Gulf Professional Publishing). 
Picciotto, M.R., Zoli, M., Rimondini, R., Léna, C., Marubio, L.M., Pich, E.M., Fuxe, K., and Changeux, J.P. (1998). Acetylcholine receptors containing the beta2 subunit are involved in the reinforcing properties of nicotine. Nature 391, 173-177.

Picciotto, M.R., and Mineur, Y.S. (2013). Molecules and circuits involved in nicotine addiction: The many faces of smoking. Neuropharmacology.

Pinault, D. (1996). A novel single-cell staining procedure performed in vivo under electrophysiological control: morpho-functional features of juxtacellularly labeled thalamic cells and other central neurons with biocytin or Neurobiotin. J. Neurosci. Methods 65, 113-136.

Poulin, J.-F., Caronia, G., Hofer, C., Cui, Q., Helm, B., Ramakrishnan, C., Chan, C.S., Dombeck, D.A., Deisseroth, K., and Awatramani, R. (2018). Mapping projections of molecularly defined dopamine neuron subtypes using intersectional genetic approaches. Nature Neuroscience 21,1-17.

Schultz, W. (2007). Multiple dopamine functions at different time courses. Annu Rev Neurosci 30, 259288.

Su, M., Li, L., Wang, J., Sun, H., Zhang, L., Zhao, C., Xie, Y., Gamper, N., Du, X., and Zhang, H. (2019). Kv7.4 Channel Contribute to Projection-Specific Auto-Inhibition of Dopamine Neurons in the Ventral Tegmental Area. Front Cell Neurosci 13, 557.

Tolu, S., Eddine, R., Marti, F., David, V., Graupner, M., Pons, S., Baudonnat, M., Husson, M., Besson, M., Reperant, C., et al. (2013). Co-activation of VTA DA and GABA neurons mediates nicotine reinforcement. Mol Psychiatry 18, 382-393.

Turiault, M., Parnaudeau, S., Milet, A., Parlato, R., Rouzeau, J.-D., Lazar, M., and Tronche, F. (2007). Analysis of dopamine transporter gene expression pattern -- generation of DAT-iCre transgenic mice. Febs J. 274, 3568-3577.

Ungless, M.A., and Grace, A.A. (2012). Are you or aren't you? Challenges associated with physiologically identifying dopamine neurons. Tins 35, 422-430.

Watabe-Uchida, M., Zhu, L., Ogawa, S.K., Vamanrao, A., and Uchida, N. (2012). Whole-Brain Mapping of Direct Inputs to Midbrain Dopamine Neurons. Neuron 74, 858-873.

Wolfman, S.L., Gill, D.F., Bogdanic, F., Long, K., Al-Hasani, R., McCall, J.G., Bruchas, M.R., and McGehee, D.S. (2018). Nicotine aversion is mediated by GABAergic interpeduncular nucleus inputs to laterodorsal tegmentum. Nature Communications 9, 2710.

Zhao-Shea, R., Liu, L., Pang, X., Gardner, P.D., and Tapper, A.R. (2013). Activation of GABAergic neurons in the interpeduncular nucleus triggers physical nicotine withdrawal symptoms. Curr Biol 23, 2327-2335.

Zhao-Shea, R., Liu, L., Soll, L.G., Improgo, M.R., Meyers, E.E., McIntosh, J.M., Grady, S.R., Marks, M.J., Gardner, P.D., and Tapper, A.R. (2011). Nicotine-mediated activation of dopaminergic neurons in distinct regions of the ventral tegmental area. Neuropsychopharmacology 36, 1021-1032.

Zhuang, X., Masson, J., Gingrich, J.A., Rayport, S., and Hen, R. (2005). Targeted gene expression in dopamine and serotonin neurons of the mouse brain. J. Neurosci. Methods 143, $27-32$. 